\title{
IMAGES OF FUNCTION AND DISTRIBUTION SPACES UNDER THE BARGMANN TRANSFORM
}

\author{
JOACHIM TOFT
}

\begin{abstract}
We consider a broad family of test function spaces and their dual (distribution) space. The family includes Gelfand-Shilov spaces, a family of test function spaces introduced by S. Pilipović. We deduce different characterizations of such spaces, especially under the Bargmann transform and the Short-time Fourier transform. The family also include a test function space, whose dual space is mapped by the Bargmann transform bijectively to the set of entire functions.
\end{abstract}

\section{INTRODUCTION}

In the paper we consider a family of test function and distribution spaces, including Gelfand-Shilov spaces. The family also include a family of functions and distribution spaces, named Pilipović test function and distribution spaces, respectively, essentially introduced by S. Pilipovic in 22 . We show that those spaces which are invariant under the Fourier transform may in convenient ways be characterized by Hermite function expansions with simple conditions on the coefficients. Furthermore we deduce that the Bargmann transform maps these spaces into topological spaces of entire functions or power series expansions, obeying convenient estimates. At the same time, characterizations of these function and distribution spaces in terms of suitable estimates of their short-time Fourier transforms with Gaussian windows are deduced.

We also introduce a test function space, whose dual, or distribution space, is mapped bijectively to the set of entire functions, by the Bargmann transform. Finally we introduce a broad family of modulation spaces as subsets of the latter distribution space, and show that these modulation spaces are quasi-Banach spaces. Here it is also shown that Gelfand-Shilov and suitable Pilipović spaces of functions and distributions are obtained by suitable unions or intersections of this broad family of modulation spaces.

One of the motivation for performing such investigations concerns questions on global and detailed regularity properties for solutions to

2010 Mathematics Subject Classification. primary 46F05; 32A25; 32A36; secondary 35Q40; 30Gxx.

Key words and phrases. Gelfand-Shilov estimates, Pilipović spaces ultradistributions, Bargmann transform. 
partial differential equations. An other motivation is to find suitable test fundtion spaces which are dense and smaller than the Schwartz space, $\mathscr{S}\left(\mathbf{R}^{d}\right)$, and which possess suitable mapping properties under the Fourier transform. The corresponding distribution spaces then become larger than the set of tempered distributions, $\mathscr{S}^{\prime}\left(\mathbf{R}^{d}\right)$, and the mapping properties for the Fourier transform carry over to these distribution spaces. For these reasons, the function and distribution spaces above might put convenient frames.

In such contexts, the Gelfand-Shilov spaces, and spaces similar to them, are often feasible. An important type of such spaces concern $\mathcal{S}_{s}\left(\mathbf{R}^{d}\right)\left(\Sigma_{s}\left(\mathbf{R}^{d}\right)\right), s \geq 0$, and consists of all smooth functions $f$ on $\mathbf{R}^{d}$ such that

$$
\sup \left|x^{\beta} D^{\alpha} f(x)\right| \lesssim h^{|\alpha+\beta|}(\alpha ! \beta !)^{s}
$$

for some (for every) $h>0$ (cf. [11]). (See [17] and Section 1 for notations.)

Evidently, $\Sigma_{s} \subseteq \mathcal{S}_{s}$ and $\mathcal{S}_{s_{0}} \subseteq \mathscr{S}$ when $s_{0} \geq 0$. If in addition $s>\frac{1}{2}$ and $s_{0} \geq \frac{1}{2}$, then these embeddings are dense, and therefore, $\mathcal{S}_{s}^{\prime} \subseteq \Sigma_{s}^{\prime}$ and $\mathscr{S}^{\prime} \subseteq \mathcal{S}_{s_{0}}^{\prime}$ hold true for corresponding distribution spaces. On the other hand, if $s \leq \frac{1}{2}$ and $s_{0}<\frac{1}{2}$, then $\Sigma_{s}$ and $\mathcal{S}_{s_{0}}$ are trivial, i. e. they are equal to $\{0\}$ (cf. [11]).

There are also several other characterizations for Gelfand-Shilov spaces. For example we have the following.

Proposition 0.1. Let $s \geq \frac{1}{2}\left(s>\frac{1}{2}\right), f \in \mathscr{S}^{\prime}\left(\mathbf{R}^{d}\right)$ and let $c_{\alpha}(f)$ be the Hermite coefficient of order $\alpha$ of $f$. Then the following conditions are equivalent:

(1) $f \in \mathcal{S}_{s}\left(\mathbf{R}^{d}\right)\left(f \in \Sigma_{s}\left(\mathbf{R}^{d}\right)\right)$;

(2) $|f(x)| \lesssim e^{-r|x|^{\frac{1}{s}}}$ and $|\widehat{f}(\xi)| \lesssim e^{-r|\xi|^{\frac{1}{s}}}$ for some (for every) $r>0$;

(3) $\left|c_{\alpha}(f)\right| \lesssim e^{-r|\alpha|^{\frac{1}{2 s}}}$ for some (for every) $r>0$.

Characterizations of the form given in Proposition 0.1 might be suitable in applications and can be carried over to other situations. For example, in [6], Dasgupta and Ruzhansky establish similar equivalences for functions defined on compact Lie groups, after the Gelfand-Shilov spaces and the conditions on $s$ are replaced by suitable Gevrey classes and $s>0$, respectively.

The equivalence between (1) and (2) in the previous proposition were deduced by Eijndhoven in [7], and later on extended by Chung, Chung and Kim in [5]. The equivalence between (1) and (3) can in an extended form be found in e.g. [19].

The Pilipović space, $\mathcal{S}_{s}\left(\mathbf{R}^{d}\right)\left(\boldsymbol{\Sigma}_{s}\left(\mathbf{R}^{d}\right)\right)^{1}$ of order $s \geq 0$, introduced in $[22]$ in the case $s \geq \frac{1}{2}$, is the set of all smooth functions $f$ on $\mathbf{R}^{d}$

\footnotetext{
${ }^{1}$ Throughout the paper it is important to distinguish between the boldface characters, $\boldsymbol{\Sigma}_{*}, \mathcal{S}_{*}$ etc. which denote Pilipović spaces, and non-boldface characters, $\Sigma_{*}$, $\mathcal{S}_{*}$ etc. which denote Gelfand-Shilov spaces. 
such that

$$
\left\|H^{N} f\right\|_{L^{\infty}} \lesssim h^{N}(N !)^{2 s}
$$

for some (for every) $h>0$, where $H=|x|^{2}-\Delta$ is the harmonic oscillator. That is, when defining $\mathcal{S}_{s}\left(\mathbf{R}^{d}\right)$ and $\boldsymbol{\Sigma}_{s}\left(\mathbf{R}^{d}\right)$, each $x_{j}$ and $D_{j}$ in (0.1), in the definition of $\mathcal{S}_{s}\left(\mathbf{R}^{d}\right)$ and $\Sigma_{s}\left(\mathbf{R}^{d}\right)$ should be replaced by $H^{\frac{1}{2}}$ in $(0.2)$.

It might not be surprising that $\mathcal{S}_{s}$ and $\boldsymbol{\Sigma}_{s}$ are similar to $\mathcal{S}_{s}$ and $\Sigma_{s}$, respectively, when taking into account the similarities in the definitions of these spaces.

In fact, we have

$$
\begin{aligned}
& \mathcal{S}_{s}\left(\mathbf{R}^{d}\right)=\mathcal{S}_{s}\left(\mathbf{R}^{d}\right), \quad \quad \text { when } \quad s \geq \frac{1}{2}, \\
& \boldsymbol{\Sigma}_{s}\left(\mathbf{R}^{d}\right)=\Sigma_{s}\left(\mathbf{R}^{d}\right), \quad \quad \text { when } \quad s>\frac{1}{2} \text { or } s=0 \text {, } \\
& \mathcal{S}_{s}\left(\mathbf{R}^{d}\right) \neq\{0\}, \mathcal{S}_{s}\left(\mathbf{R}^{d}\right)=\{0\}, \text { when } \quad s<\frac{1}{2},
\end{aligned}
$$

and

$$
\boldsymbol{\Sigma}_{s}\left(\mathbf{R}^{d}\right) \neq\{0\}, \Sigma_{s}\left(\mathbf{R}^{d}\right)=\{0\}, \quad \text { when } \quad s \leq \frac{1}{2}, s \neq 0 .
$$

Here (0.6) in the case $s=\frac{1}{2},(0.3)$ and (0.4) was proved already in [22]. Furthermore, the arguments in [22] also show that (0.5) and (0.6) hold for any admissible $s$, since $\mathcal{S}_{s}\left(\mathbf{R}^{d}\right)$ and $\boldsymbol{\Sigma}_{s}\left(\mathbf{R}^{d}\right)$ contain all Hermite functions on $\mathbf{R}^{d}$ for such choices of $s$.

The set $\boldsymbol{\Sigma}\left(\mathbf{R}^{d}\right)=\boldsymbol{\Sigma}_{\frac{1}{2}}\left(\mathbf{R}^{d}\right)$ is of peculiar interests, because it is the largest test function space among the sets $\boldsymbol{\Sigma}_{s}\left(\mathbf{R}^{d}\right)$ and $\mathcal{S}_{s}\left(\mathbf{R}^{d}\right)$ which at the same time is not a Gelfand-Shilov space.

In Section 2 we introduce a family of test function spaces, denoted by $\mathcal{H}_{s}\left(\mathbf{R}^{d}\right)$ and $\mathcal{H}_{0, s}\left(\mathbf{R}^{d}\right), s \geq 0$, and contains all smooth functions $f$ on $\mathbf{R}^{d}$ such that its Hermite coefficients satisfy

$$
\left|c_{\alpha}(f)\right| \lesssim e^{-r|\alpha|^{\frac{1}{2 s}}}
$$

for some (for every) $r>0$. By Proposition 0.1 it follows that $\mathcal{H}_{s}=\mathcal{S}_{s}$ when $s \geq \frac{1}{2}$ and $\mathcal{H}_{0, s}=\Sigma_{s}$ when $s>\frac{1}{2}$. In Section 4 we extend these relations into $\mathcal{H}_{s}=\mathcal{S}_{s}$ and $\mathcal{H}_{0, s}=\boldsymbol{\Sigma}_{s}$ for every $s>0$.

In Section 2 we also consider test function spaces, denoted by $\mathcal{H}_{b_{\sigma}}\left(\mathbf{R}^{d}\right)$ and $\mathcal{H}_{0, b_{\sigma}}\left(\mathbf{R}^{d}\right), \sigma>0$, where the assumptions on the Hermite coefficients above are replaced by

$$
\left|c_{\alpha}(f)\right| \lesssim r^{|\alpha|}(\alpha !)^{-\frac{1}{2 \sigma}} .
$$

It follows that these spaces contains any $\mathcal{S}_{s}$ when $s<\frac{1}{2}$, but are smaller and dense in $\boldsymbol{\Sigma}\left(\mathbf{R}^{d}\right)$. Hence, their duals $\mathcal{H}_{b_{\sigma}}^{\prime}\left(\mathbf{R}^{d}\right)$ and $\mathcal{H}_{0, b_{\sigma}}^{\prime}\left(\mathbf{R}^{d}\right)$, respectively, contain $\boldsymbol{\Sigma}^{\prime}\left(\mathbf{R}^{d}\right)$. 
A motivations for considering these spaces is the convenient mapping properties of the Bargmann transform on these spaces. More precisely, in Section 3 we deduce that any set of entire functions

$$
\begin{aligned}
& \left\{F \in A\left(\mathbf{C}^{d}\right) ;|F(z)| \lesssim e^{r|z|^{\tau}} \text { for every } r>0\right\}, \\
& \left\{F \in A\left(\mathbf{C}^{d}\right) ;|F(z)| \lesssim e^{r|z|^{\tau}} \text { for some } r>0\right\},
\end{aligned}
$$

when $\tau>0$, as well as $A\left(\mathbf{C}^{d}\right)$, and the set of all functions which are defined and analytic near origin in $\mathbf{C}^{d}$, are counter images of the spaces

$$
\begin{array}{ll}
\mathcal{H}_{0, b_{\sigma}}\left(\mathbf{R}^{d}\right), & \mathcal{H}_{b_{\sigma}}\left(\mathbf{R}^{d}\right), \quad \mathcal{H}_{0, \frac{1}{2}}\left(\mathbf{R}^{d}\right) \\
\mathcal{H}_{0, \frac{1}{2}}^{\prime}\left(\mathbf{R}^{d}\right), & \mathcal{H}_{b_{\sigma}}^{\prime}\left(\mathbf{R}^{d}\right) \quad \text { and } \quad \mathcal{H}_{0, b_{\sigma}}^{\prime}\left(\mathbf{R}^{d}\right),
\end{array}
$$

for suitable choices of $\sigma$. (See Theorem 3.1 or the tables in Section 6.)

More precisely, beside

$$
\mathcal{H}_{0, s}\left(\mathbf{R}^{d}\right), \quad \mathcal{H}_{s}\left(\mathbf{R}^{d}\right), \quad \mathcal{H}_{s}^{\prime}\left(\mathbf{R}^{d}\right), \quad \mathcal{H}_{0, s}^{\prime}\left(\mathbf{R}^{d}\right), \quad s \geq 0 \text { or } s=b_{\sigma},
$$

we also introduce

$$
\mathcal{A}_{0, s}\left(\mathbf{C}^{d}\right), \quad \mathcal{A}_{s}\left(\mathbf{C}^{d}\right), \quad \mathcal{A}_{s}^{\prime}\left(\mathbf{C}^{d}\right), \quad \mathcal{A}_{0, s}^{\prime}\left(\mathbf{C}^{d}\right), \quad s \geq 0 \text { or } s=b_{\sigma},
$$

of suitable topological spaces of power series on $\mathbf{C}^{d}$, in Section 2, From the definitions it follows that the Bargmann transform is homeomorphic from the former spaces to the latter ones. Hence, the duals of $\mathcal{A}_{0, s}\left(\mathbf{C}^{d}\right)$ and $\mathcal{A}_{s}\left(\mathbf{C}^{d}\right)$ can be identified with $\mathcal{A}_{0, s}^{\prime}\left(\mathbf{C}^{d}\right)$ and $\mathcal{A}_{s}^{\prime}\left(\mathbf{C}^{d}\right)$, respectively. In Sections 3 and 4 we show that the spaces in (0.9) are convenient spaces of entire functions, e. g. of the form (0.7). A separate case appears when $s<\frac{1}{2}$, and in this situation the estimates on $F$ in (0.7) should be replaced by

$$
|F(z)| \lesssim e^{r\left(\log \langle z\rangle^{\frac{1}{1-2 s}}\right.}
$$

(cf. [9, Theorem 10]). We refer to the tables in the end of Section 6] for a more comprehensive explanation of these image properties.

In the second part of Section 3 we show that $\mathcal{H}_{b_{1}}^{\prime}$ might be feasible when defining broad families of modulation spaces, with minimal restrictions on the involved weight functions. In fact, it is here proved that if the modulation spaces are defined in the background of $\mathcal{H}_{b_{1}}^{\prime}$, then these spaces are complete. In Section 3 we also deduce that the test function space $\mathcal{S}_{C}$, introduced by Gröchenig in [15], agrees with $\mathcal{H}_{b_{1}}$.

In the same manner we consider the Gelfand-Shilov spaces $\mathcal{S}_{t_{0}}^{s_{0}}\left(\mathbf{R}^{d}\right)$ and $\Sigma_{t}^{s}\left(\mathbf{R}^{d}\right)$, and their distribution spaces $\left(\mathcal{S}_{t_{0}}^{s_{0}}\right)^{\prime}\left(\mathbf{R}^{d}\right)$ and $\left(\Sigma_{t}^{s}\right)^{\prime}\left(\mathbf{R}^{d}\right)$. These spaces and the Pilipović spaces, are related to each others by 
the relations $(\underline{0.3})-(\underline{0.6})$, and

$$
\begin{aligned}
\Sigma\left(\mathbf{R}^{d}\right) \subseteq \mathcal{S}_{t_{0}}^{s_{0}}\left(\mathbf{R}^{d}\right) \subseteq \Sigma_{t}^{s}\left(\mathbf{R}^{d}\right) \subseteq \mathcal{S}_{t}^{s}\left(\mathbf{R}^{d}\right) \subseteq \mathscr{S}\left(\mathbf{R}^{d}\right) \\
\subseteq \mathscr{S}^{\prime}\left(\mathbf{R}^{d}\right) \subseteq\left(\mathcal{S}_{t}^{s}\right)^{\prime}\left(\mathbf{R}^{d}\right) \subseteq\left(\Sigma_{t}^{s}\right)^{\prime}\left(\mathbf{R}^{d}\right) \subseteq\left(\mathcal{S}_{t_{0}}^{s_{0}}\right)^{\prime}\left(\mathbf{R}^{d}\right) \subseteq \Sigma^{\prime}\left(\mathbf{R}^{d}\right), \\
\text { when } \quad \frac{1}{2} \leq s_{0}<s \text { and } \frac{1}{2} \leq t_{0}<t, \quad(0 .
\end{aligned}
$$

and

$$
\boldsymbol{\Sigma}_{s_{1}}\left(\mathbf{R}^{d}\right) \subseteq \mathcal{S}_{s_{1}}\left(\mathbf{R}^{d}\right) \subseteq \boldsymbol{\Sigma}_{s_{2}}\left(\mathbf{R}^{d}\right) \quad \text { when } \quad s_{1}<s_{2} .
$$

In Section 5 we describe the images of $\Sigma_{t}^{s}, \mathcal{S}_{t}^{s}$ and their duals under the Bargmann transform, for suitable $s$ and $t$. (See also [4] for sub results.) For example, since $\mathcal{H}_{s}\left(\mathbf{R}^{d}\right)=\mathcal{S}_{s}\left(\mathbf{R}^{d}\right)$ when $s \geq \frac{1}{2}$, it follows from the image properties of the Bargmann properties on Pilipović spaces that the mappings

$$
\begin{aligned}
& \mathfrak{V}_{d}: \mathcal{S}_{s}\left(\mathbf{R}^{d}\right) \rightarrow \mathcal{A}_{s}\left(\mathbf{C}^{d}\right) \\
& \mathfrak{V}_{d}: \mathcal{S}_{s}^{\prime}\left(\mathbf{R}^{d}\right) \rightarrow \mathcal{A}_{s}^{\prime}\left(\mathbf{C}^{d}\right)
\end{aligned}
$$

are bijective, which in terms of the the characterization results in Sections 3 and 4 can be described as.

$$
\mathcal{A}_{s}\left(\mathbf{C}^{d}\right)=\left\{F \in A\left(\mathbf{C}^{d}\right) ;|F(z)| \lesssim e^{\frac{|z|^{2}}{2}-r|z|^{\frac{1}{s}}}, \text { for some } r>0\right\}
$$

and

$$
\mathcal{A}_{s}^{\prime}\left(\mathbf{C}^{d}\right)=\left\{F \in A\left(\mathbf{C}^{d}\right) ;|F(z)| \lesssim e^{\frac{|z|^{2}}{2}+r|z|^{\frac{1}{s}}}, \text { for every } r>0\right\},
$$

for such choices of $s$. In Section 5 we, more generally, characterize all the Gelfand-Shilov spaces of functions and distributions in (0.10) in such ways (cf. Theorem 5.2).

Since short-time Fourier transforms with the standard Gaussian as window functions carry over to corresponding Bargmann transforms by simple manipulations, the mapping results for the Bargmann transform also lead to characterizations of Gelfand-Shilov spaces, Pilipović spaces and their duals in terms of estimates on the short-time Fourier transforms (see Remark 4.6 and Proposition 6.5). For example, let $V_{\phi} f$ be the short-time Fourier transform of $f$ with respect to the standard Gaussian function $\phi$ as the window function. Then it follows from (0.13), (0.14) and corresponding mapping properties for $\boldsymbol{\Sigma}_{s}\left(\mathbf{R}^{d}\right)$ and $\boldsymbol{\Sigma}_{s}^{\prime}\left(\mathbf{R}^{d}\right)$ that if $s=\frac{1}{2}$, then $f \in \mathcal{S}_{s}\left(\mathbf{R}^{d}\right)\left(f \in \boldsymbol{\Sigma}_{s}\left(\mathbf{R}^{d}\right)\right)$, if and only if

$$
\left|V_{\phi} f(x, \xi)\right| \lesssim e^{-\frac{|x|^{2}+|\xi|^{2}}{4(1+r)}},
$$

for some (for every) $r>0$, and $f \in \mathcal{S}_{s}^{\prime}\left(\mathbf{R}^{d}\right)\left(f \in \boldsymbol{\Sigma}_{s}^{\prime}\left(\mathbf{R}^{d}\right)\right)$, if and only if

$$
\left|V_{\phi} f(x, \xi)\right| \underset{5}{\lesssim} e^{\frac{r\left(|x|^{2}+|\xi|^{2}\right)}{4}}
$$


for every (for some) $r>0$ (see Proposition 6.5).

In Section 6 we present some consequences and further remarks. It is here proved that every Pilipović space are invariant under fractional Fourier transforms. Here it is also shown that Pilipović spaces which are not Gelfand-Shilov spaces are neither invariant under dilations nor algebras. Evidently, such properties are important when discussing problems for partial differential equations, and it is expected that the absence of such properties have bad impact on the applicability of such spaces. On the other hand, in Section 6 we also deduce that the Pilipović spaces are invariant under actions of linear partial differential operators with polynomial coefficients. Hence, it makes sense to discuss this class of operators in the framework of Pilipović spaces. Note that most of operators which appear in physics and engineering belong to this class.

In Section 6 we also give examples on extension of the notion of Pilipović spaces in terms of counter images of suitable spaces of entire functions under the Bargmann transform. For example, we give a definition of spaces $\boldsymbol{\Sigma}_{t}^{s}\left(\mathbf{R}^{d}\right)$ and $\mathcal{S}_{t}^{s}\left(\mathbf{R}^{d}\right)$ when $s \geq 0$ or $s=b_{\sigma}$ and $t \geq 0$ or $t=b_{\sigma}$ such that $\boldsymbol{\mathcal { S }}_{t}^{s}=\mathcal{S}_{t}^{s}$ when $s, t \geq \frac{1}{2}, \boldsymbol{\Sigma}_{t}^{s}=\Sigma_{t}^{s}$ when $s, t>\frac{1}{2}$, and $\Sigma_{s}^{s}=\Sigma_{s}, \mathcal{S}_{s}^{s}=\mathcal{S}_{s}$ for general $s$.

\section{ACKNOWLEDGEMENT}

I am very grateful to professors Stevan Pilipović and Nenad Teofanov for careful reading of the paper, leading to improvements of the content and the style. I am also grateful to Yuanyuan Chen and Patrik Wahlberg for fruitful and valuable discussions.

\section{Preliminaries}

In this section we recall some basic facts. We start by discussing Gelfand-Shilov spaces and their properties. Thereafter we recall the definition of Pilipović spaces and some of their properties. Then we recall some facts on modulation spaces. Finally we recall the Bargmann transform and some of its mapping properties, and introduce suitable classes of entire functions on $\mathbf{C}^{d}$.

1.1. Gelfand-Shilov spaces. We start by recalling some facts on Gelfand-Shilov spaces. Let $0<h, s, t \in \mathbf{R}$ be fixed. Then $\mathcal{S}_{s, h}\left(\mathbf{R}^{d}\right)$ consists of all $f \in C^{\infty}\left(\mathbf{R}^{d}\right)$ such that

$$
\|f\|_{\mathcal{S}_{t, h}^{s}} \equiv \sup \frac{\left|x^{\beta} \partial^{\alpha} f(x)\right|}{h^{|\alpha+\beta|} \alpha !^{!} \beta !^{t}}
$$

is finite. Here the supremum should be taken over all $\alpha, \beta \in \mathbf{N}^{d}$ and $x \in \mathbf{R}^{d}$.

Obviously $\mathcal{S}_{s, h}^{t}$ is a Banach space, contained in $\mathscr{S}$, and which increases with $h, s$ and $t$ and $\mathcal{S}_{s, h}^{t} \hookrightarrow \mathscr{S}$. Here and in what follows we 
use the notation $A \hookrightarrow B$ when the topological spaces $A$ and $B$ satisfy $A \subseteq B$ with continuous embeddings. Furthermore, if $s, t>\frac{1}{2}$, or $s=t=\frac{1}{2}$ and $h$ is sufficiently large, then $\mathcal{S}_{t, h}^{s}$ contains all finite linear combinations of Hermite functions. Since such linear combinations are dense in $\mathscr{S}$ and in $\mathcal{S}_{t, h}^{s}$, it follows that the dual $\left(\mathcal{S}_{t, h}^{s}\right)^{\prime}\left(\mathbf{R}^{d}\right)$ of $\mathcal{S}_{t, h}^{s}\left(\mathbf{R}^{d}\right)$ is a Banach space which contains $\mathscr{S}^{\prime}\left(\mathbf{R}^{d}\right)$, for such choices of $s$ and $t$.

The Gelfand-Shilov spaces $\mathcal{S}_{t}^{s}\left(\mathbf{R}^{d}\right)$ and $\Sigma_{t}^{s}\left(\mathbf{R}^{d}\right)$ are defined as the inductive and projective limits respectively of $\mathcal{S}_{t, h}^{s}\left(\mathbf{R}^{d}\right)$. This implies that

$$
\mathcal{S}_{t}^{s}\left(\mathbf{R}^{d}\right)=\bigcup_{h>0} \mathcal{S}_{t, h}^{s}\left(\mathbf{R}^{d}\right) \quad \text { and } \quad \Sigma_{t}^{s}\left(\mathbf{R}^{d}\right)=\bigcap_{h>0} \mathcal{S}_{t, h}^{s}\left(\mathbf{R}^{d}\right),
$$

and that the topology for $\mathcal{S}_{t}^{s}\left(\mathbf{R}^{d}\right)$ is the strongest possible one such that the inclusion map from $\mathcal{S}_{t, h}^{s}\left(\mathbf{R}^{d}\right)$ to $\mathcal{S}_{t}^{s}\left(\mathbf{R}^{d}\right)$ is continuous, for every choice of $h>0$. The space $\Sigma_{t}^{s}\left(\mathbf{R}^{d}\right)$ is a Fréchet space with seminorms $\|\cdot\|_{\mathcal{S}_{t, h}^{s}}, h>0$. Moreover, $\Sigma_{t}^{s}\left(\mathbf{R}^{d}\right) \neq\{0\}$, if and only if $s+t \geq 1$ and $(s, t) \neq\left(\frac{1}{2}, \frac{1}{2}\right)$, and $\mathcal{S}_{t}^{s}\left(\mathbf{R}^{d}\right) \neq\{0\}$, if and only if $s+t \geq 1$.

The Gelfand-Shilov distribution spaces $\left(\mathcal{S}_{t}^{s}\right)^{\prime}\left(\mathbf{R}^{d}\right)$ and $\left(\Sigma_{t}^{s}\right)^{\prime}\left(\mathbf{R}^{d}\right)$ are the projective and inductive limit respectively of $\left(\mathcal{S}_{t, h}^{s}\right)^{\prime}\left(\mathbf{R}^{d}\right)$. This means that

$$
\left(\mathcal{S}_{t}^{s}\right)^{\prime}\left(\mathbf{R}^{d}\right)=\bigcap_{h>0}\left(\mathcal{S}_{t, h}^{s}\right)^{\prime}\left(\mathbf{R}^{d}\right) \quad \text { and } \quad\left(\Sigma_{t}^{s}\right)^{\prime}\left(\mathbf{R}^{d}\right)=\bigcup_{h>0}\left(\mathcal{S}_{t, h}^{s}\right)^{\prime}\left(\mathbf{R}^{d}\right) . \quad(1.2)^{\prime}
$$

We remark that in [11] it is proved that $\left(\mathcal{S}_{t}^{s}\right)^{\prime}\left(\mathbf{R}^{d}\right)$ is the dual of $\mathcal{S}_{t}^{s}\left(\mathbf{R}^{d}\right)$, and $\left(\Sigma_{t}^{s}\right)^{\prime}\left(\mathbf{R}^{d}\right)$ is the dual of $\Sigma_{t}^{s}\left(\mathbf{R}^{d}\right)$ (also in topological sense). For conveniency we set

$$
\mathcal{S}_{s}=\mathcal{S}_{s}^{s}, \quad \mathcal{S}_{s}^{\prime}=\left(\mathcal{S}_{s}^{s}\right)^{\prime}, \quad \Sigma_{s}=\Sigma_{s}^{s} \quad \text { and } \quad \Sigma_{s}^{\prime}=\left(\Sigma_{s}^{s}\right)^{\prime} .
$$

For every admissible $s, t>0$ and $\varepsilon>0$ we have

$$
\begin{aligned}
& \Sigma_{t}^{s}\left(\mathbf{R}^{d}\right) \hookrightarrow \mathcal{S}_{t}^{s}\left(\mathbf{R}^{d}\right) \hookrightarrow \Sigma_{t+\varepsilon}^{s+\varepsilon}\left(\mathbf{R}^{d}\right) \\
& \text { and } \quad\left(\Sigma_{t+\varepsilon}^{s+\varepsilon}\right)^{\prime}\left(\mathbf{R}^{d}\right) \hookrightarrow\left(\mathcal{S}_{t}^{s}\right)^{\prime}\left(\mathbf{R}^{d}\right) \hookrightarrow\left(\Sigma_{t}^{s}\right)^{\prime}\left(\mathbf{R}^{d}\right) .
\end{aligned}
$$

A convenient basis for Gelfand-Shilov spaces on $\mathbf{R}^{d}$ concerns the set of Hermite functions, $\left\{h_{\alpha}\right\}_{\alpha \in \mathbf{N}^{d}}$ on $\mathbf{R}^{d}$, where the Hermite function $h_{\alpha}$ of order $\alpha \in \mathbf{N}^{d}$ is defined by

$$
h_{\alpha}(x)=\pi^{-\frac{d}{4}}(-1)^{|\alpha|}\left(2^{|\alpha|} \alpha !\right)^{-\frac{1}{2}} e^{\frac{|x|^{2}}{2}}\left(\partial^{\alpha} e^{-|x|^{2}}\right) .
$$

It follows that

$$
h_{\alpha}(x)=\left((2 \pi)^{\frac{d}{2}} \alpha !\right)^{-1} e^{-\frac{|x|^{2}}{2}} p_{\alpha}(x),
$$

for some polynomial $p_{\alpha}$ on $\mathbf{R}^{d}$, which is called the Hermite polynomial of order $\alpha$.

The set $\left\{h_{\alpha}\right\}_{\alpha \in \mathbf{N}^{d}}$ is an orthonormal basis for $L^{2}\left(\mathbf{R}^{d}\right)$. It is also a basis for the Schwartz space and its distribution space, and for any $\Sigma_{t}^{s}$ when $s, t>\frac{1}{2}, \mathcal{S}_{t}^{s}$ when $s, t \geq \frac{1}{2}$ and their distribution spaces. They 
are also eigenfunctions to the Harmonic oscillator $H \equiv|x|^{2}-\Delta$. More precisely, we have

$$
H h_{\alpha}=(2|\alpha|+d) h_{\alpha}, \quad H \equiv|x|^{2}-\Delta .
$$

From now on we let $\mathscr{F}$ be the Fourier transform which takes the form

$$
(\mathscr{F} f)(\xi)=\widehat{f}(\xi) \equiv(2 \pi)^{-\frac{d}{2}} \int_{\mathbf{R}^{d}} f(x) e^{-i\langle x, \xi\rangle} d x
$$

when $f \in L^{1}\left(\mathbf{R}^{d}\right)$. Here $\langle\cdot, \cdot\rangle$ denotes the usual scalar product on $\mathbf{R}^{d}$. The map $\mathscr{F}$ extends uniquely to homeomorphisms on $\mathscr{S}^{\prime}\left(\mathbf{R}^{d}\right)$, from $\left(\mathcal{S}_{t}^{s}\right)^{\prime}\left(\mathbf{R}^{d}\right)$ to $\left(\mathcal{S}_{s}^{t}\right)^{\prime}\left(\mathbf{R}^{d}\right)$ and from $\left(\Sigma_{t}^{s}\right)^{\prime}\left(\mathbf{R}^{d}\right)$ to $\left(\Sigma_{s}^{t}\right)^{\prime}\left(\mathbf{R}^{d}\right)$. Furthermore, $\mathscr{F}$ restricts to homeomorphisms on $\mathscr{S}\left(\mathbf{R}^{d}\right)$, from $\mathcal{S}_{t}^{s}\left(\mathbf{R}^{d}\right)$ to $\mathcal{S}_{s}^{t}\left(\mathbf{R}^{d}\right)$ and from $\Sigma_{t}^{s}\left(\mathbf{R}^{d}\right)$ to $\Sigma_{s}^{t}\left(\mathbf{R}^{d}\right)$, and to a unitary operator on $L^{2}\left(\mathbf{R}^{d}\right)$. Similar facts hold true when $s=t$ and the Fourier transform is replaced by a partial Fourier transform.

Gelfand-Shilov spaces and their distribution spaces can in convenient ways be characterized by means of estimates of short-time Fourier transforms, (see e.g. [16, 26]). We here recall the details and start by giving the definition of the short-time Fourier transform.

Let $\phi \in \mathcal{S}_{s}^{\prime}\left(\mathbf{R}^{d}\right)$ be fixed. Then the short-time Fourier transform $V_{\phi} f$ of $f \in \mathcal{S}_{s}^{\prime}\left(\mathbf{R}^{d}\right)$ with respect to the window function $\phi$ is the GelfandShilov distribution on $\mathbf{R}^{2 d}$, defined by

$$
V_{\phi} f(x, \xi) \equiv\left(\mathscr{F}_{2}(U(f \otimes \phi))\right)(x, \xi)=\mathscr{F}(f \overline{\phi(\cdot-x)})(\xi),
$$

where $(U F)(x, y)=F(y, y-x)$. Here $\mathscr{F}_{2} F$ is the partial Fourier transform of $F(x, y) \in \mathcal{S}_{s}^{\prime}\left(\mathbf{R}^{2 d}\right)$ with respect to the $y$ variable. If $f, \phi \in$ $\mathcal{S}_{s}\left(\mathbf{R}^{d}\right)$, then it follows that

$$
V_{\phi} f(x, \xi)=(2 \pi)^{-\frac{d}{2}} \int f(y) \overline{\phi(y-x)} e^{-i\langle y, \xi\rangle} d y .
$$

In the sequel, $A \lesssim B$ means that $A \leq c B$ for a suitable constant $c>0$. We also set $A \asymp B$ when $A \lesssim B$ and $B \lesssim A$.

Proposition 1.1. Let $s, t, s_{0}, t_{0}>0$ be such that $s_{0}+t_{0} \geq 1, s_{0} \leq s$ and $t_{0} \leq t$. Also let $\phi \in \mathcal{S}_{t_{0}}^{s_{0}}\left(\mathbf{R}^{d}\right) \backslash 0$ and $f \in\left(\mathcal{S}_{t_{0}}^{s_{0}}\right)^{\prime}\left(\mathbf{R}^{d}\right)$. Then the following is true:

(1) $f \in \mathcal{S}_{t}^{s}\left(\mathbf{R}^{d}\right)$, if and only if

$$
\left|V_{\phi} f(x, \xi)\right| \lesssim e^{-r\left(|x|^{\frac{1}{t}}+|\xi|^{\frac{1}{s}}\right)},
$$

holds for some $r>0$;

(2) if in addition $\left(s_{0}, t_{0}\right) \neq\left(\frac{1}{2}, \frac{1}{2}\right)$ and $\phi \in \Sigma_{t_{0}}^{s_{0}}\left(\mathbf{R}^{d}\right)$, then $f \in$ $\Sigma_{t}^{s}\left(\mathbf{R}^{d}\right)$, if and only if (1.5) holds for every $r>0$.

A proof of Theorem 1.1 can be found in e.g. [16] (cf. [16, Theorem 2.7]). The corresponding result for Gelfand-Shilov distributions is the following improvement of [26, Theorem 2.5]. 
Proposition 1.2. Let $s, t, s_{0}, t_{0}>0$ be such that $s_{0}+t_{0} \geq 1, s_{0} \leq s$ and $t_{0} \leq t$. Also let $\phi \in \mathcal{S}_{t}^{s}\left(\mathbf{R}^{d}\right) \backslash 0$ and $f \in\left(\mathcal{S}_{t_{0}}^{s_{0}}\right)^{\prime}\left(\mathbf{R}^{d}\right)$. Then the following is true:

(1) $f \in\left(\mathcal{S}_{t}^{s}\right)^{\prime}\left(\mathbf{R}^{d}\right)$, if and only if

$$
\left|V_{\phi} f(x, \xi)\right| \lesssim e^{r\left(|x|^{\frac{1}{t}}+|\xi|^{\frac{1}{s}}\right)}
$$

holds for every $r>0$;

(2) if in addition $\left(s_{0}, t_{0}\right) \neq\left(\frac{1}{2}, \frac{1}{2}\right)$ and $\phi \in \Sigma_{t_{0}}^{s_{0}}\left(\mathbf{R}^{d}\right)$, then $f \in$ $\left(\Sigma_{t}^{s}\right)^{\prime}\left(\mathbf{R}^{d}\right)$, if and only if (1.5) holds for some $r>0$.

We note that in (2) in [26, Theorem 2.5] it should stay $\left(\Sigma_{t}^{s}\right)^{\prime}\left(\mathbf{R}^{d}\right)$ instead of $\Sigma_{t}^{s}\left(\mathbf{R}^{d}\right)$. In Section 5 we show that Proposition 1.2 (1) remains true also in the case $s=t=\frac{1}{2}$ and $\phi$ is a Gaussian.

Proof. The assertion (2) is the same as (2) in [26, Theorem 2.5].

Let $p \in[1, \infty]$ and let $Q_{r}^{p}\left(\mathbf{R}^{d}\right)$ be the set of all $\phi \in L_{l o c}^{p}\left(\mathbf{R}^{d}\right)$ such that

$$
\|\phi\|_{Q_{r}^{p}} \equiv\left\|\phi e^{r|\cdot| \frac{1}{t}}\right\|_{L^{p}}+\left\|\widehat{\phi} e^{r|\cdot|^{\frac{1}{s}}}\right\|_{L^{p}}
$$

is finite. By [5,7] it follows that $\mathcal{S}_{t}^{s}\left(\mathbf{R}^{d}\right)$ is the inductive limit of $Q_{r}^{p}\left(\mathbf{R}^{d}\right)$ with respect to $r>0$, also in topological sense. Hence, if $f \in\left(\mathcal{S}_{t}^{s}\right)^{\prime}\left(\mathbf{R}^{d}\right)$, then

for every $r>0$.

$$
|(f, \phi)| \lesssim\|\phi\|_{Q_{r}^{p},} \quad \phi \in \mathcal{S}_{t}^{s}\left(\mathbf{R}^{d}\right)
$$

Now assume that $\phi \in \mathcal{S}_{t}^{s}\left(\mathbf{R}^{d}\right)$ is fixed. Then there is an $r_{0}>0$ such that $\phi \in Q_{\varepsilon}^{p}\left(\mathbf{R}^{d}\right)$ for every $\varepsilon \in\left(0, r_{0}\right]$. Hence, for such $\varepsilon$ we have

$$
\begin{aligned}
\left|V_{\phi} f(x, \xi)\right|= & \left|\left(f, \phi(\cdot-x) e^{i<\cdot, \xi\rangle}\right)\right| \\
& \\
& \left\|\phi(\cdot-x) e^{\varepsilon|\cdot| \frac{1}{t}}\right\|_{L^{p}}+\left\|\widehat{\phi}(\cdot-\xi) e^{\varepsilon|\cdot|^{\frac{1}{s}}}\right\|_{L^{p}} \\
& \left.\lesssim e^{\varepsilon c|x|^{\frac{1}{t}}}+e^{\varepsilon c|\xi|^{\frac{1}{s}}} \lesssim e^{\varepsilon c\left(|x|^{\frac{1}{t}}+|\xi|^{\frac{1}{s}}\right.}\right)
\end{aligned}
$$

which implies that (1.5) holds for every $r>0$.

On the other hand, assume that (1.5) holds for every $r>0$, and let $\psi \in \mathcal{S}_{t_{0}}^{s_{0}}\left(\mathbf{R}^{d}\right)$. By Moyal's identity

$$
(f, \psi)=\|\phi\|_{L^{2}}^{-2}\left(V_{\phi} f, V_{\phi} \psi\right),
$$

Proposition 1.1 and (1.5), and the fact that $\mathcal{S}_{t_{0}}^{s_{0}}$ is dense in $\mathcal{S}_{t}^{s}$, it follows that the $(f, \psi)$ extends uniquely to any $\psi \in \mathcal{S}_{t}^{s}\left(\mathbf{R}^{d}\right)$, and that

$$
|(f, \psi)| \lesssim\|\psi\|_{Q_{r}^{\infty}}
$$

for every $r>0$. Hence, $f \in\left(\mathcal{S}_{t}^{s}\right)^{\prime}\left(\mathbf{R}^{d}\right)$, which gives the result.

Remark 1.3. The short-time Fourier transform can also be used to identify elements in $\mathscr{S}\left(\mathbf{R}^{d}\right)$ and in $\mathscr{S}^{\prime}\left(\mathbf{R}^{d}\right)$. In fact, if $\phi \in \mathscr{S}\left(\mathbf{R}^{d}\right) \backslash 0$ and $f \in\left(\mathcal{S}_{\frac{1}{2}}\right)^{\prime}\left(\mathbf{R}^{d}\right)$, then the following is true: 
(1) $f \in \mathscr{S}\left(\mathbf{R}^{d}\right)$, if and only if for every $N \geq 0$, it holds

$$
\left|V_{\phi} f(x, \xi)\right| \lesssim\langle x, \xi\rangle^{-N}
$$

(2) $f \in \mathscr{S}^{\prime}\left(\mathbf{R}^{d}\right)$, if and only if for some $N \geq 0$, it holds

$$
\left|V_{\phi} f(x, \xi)\right| \lesssim\langle x, \xi\rangle^{N}
$$

(Cf. [15, Chapter 12].)

1.2. The Pilipović spaces. Next we consider spaces which are obtained by suitable estimates of Gelfand-Shilov or Gevrey type, after the operator $x^{\beta} \partial^{\alpha}$ in (1.1) is replaced by powers of the harmonic oscillator $H=|x|^{2}-\Delta$. If $s_{0} \geq \frac{1}{2}$ and $s>\frac{1}{2}$, then it turns out that this is merely an alternative approach for obtaining the Gelfand-Shilov spaces $\mathcal{S}_{s_{0}}\left(\mathbf{R}^{d}\right)$ and $\Sigma_{s}\left(\mathbf{R}^{d}\right)$, respectively. (Cf. [12,21,22].)

Let $h>0, s \geq 0$ and let $\mathcal{S}_{h, s}\left(\mathbf{R}^{d}\right)$ be the set of all $f \in C^{\infty}\left(\mathbf{R}^{d}\right)$ such that

$$
\|f\|_{\mathcal{S}_{h, s}} \equiv \sup _{N \geq 0} \frac{\left\|H^{N} f\right\|_{L^{\infty}}}{h^{N}(N !)^{2 s}}<\infty .
$$

Then $\mathcal{S}_{h, s}\left(\mathbf{R}^{d}\right)$ is a Banach space. If $s>0$, then $\mathcal{S}_{h, s}\left(\mathbf{R}^{d}\right)$ contains all Hermite functions. Furthermore, if $s=0$, and $\alpha \in \mathbf{N}^{d}$ satisfies $2|\alpha|+d \leq h$, then $h_{\alpha} \in \mathcal{S}_{h, s}\left(\mathbf{R}^{d}\right)$.

We let

$$
\boldsymbol{\Sigma}_{s}\left(\mathbf{R}^{d}\right) \equiv \bigcap_{h>0} \mathcal{S}_{h, s}\left(\mathbf{R}^{d}\right) \quad \text { and } \quad \mathcal{S}_{s}\left(\mathbf{R}^{d}\right) \equiv \bigcup_{h>0} \mathcal{S}_{h, s}\left(\mathbf{R}^{d}\right),
$$

and equip these spaces by projective and inductive limit topologies, respectively, of $\mathcal{S}_{h, s}\left(\mathbf{R}^{d}\right), h>0$.

In [21,22], Pilipović proved

$$
\mathcal{S}_{s}\left(\mathbf{R}^{d}\right)=\mathcal{S}_{s}\left(\mathbf{R}^{d}\right), \quad s \geq \frac{1}{2}, \quad \text { and } \quad \Sigma_{s}\left(\mathbf{R}^{d}\right)=\boldsymbol{\Sigma}_{s}\left(\mathbf{R}^{d}\right), \quad s>\frac{1}{2} .
$$

On the other hand, $\Sigma_{s}\left(\mathbf{R}^{d}\right)$ is trivially equal to $\{0\}$ when $s \leq \frac{1}{2}$, while any Hermite function $h_{\alpha}$ fulfills (1.6) for every $h>0$, when $0<s \leq \frac{1}{2}$.

Hence,

$$
\Sigma_{s}\left(\mathbf{R}^{d}\right)=\Sigma_{s}\left(\mathbf{R}^{d}\right), \quad s>\frac{1}{2}
$$

and

$$
\boldsymbol{\Sigma}_{s}\left(\mathbf{R}^{d}\right) \neq \Sigma_{s}\left(\mathbf{R}^{d}\right)=\emptyset \quad 0<s \leq \frac{1}{2} .
$$

We call $\boldsymbol{\Sigma}_{s}\left(\mathbf{R}^{d}\right)$ the Pilipović space (of Beurling type) of order $s \geq 0$ on $\mathbf{R}^{d}$. For conveniency we set $\boldsymbol{\Sigma}\left(\mathbf{R}^{d}\right) \equiv \Sigma_{\frac{1}{2}}\left(\mathbf{R}^{d}\right)$, and call this space the Pilipovic space on $\mathbf{R}^{d}$.

Similarly, $\mathcal{S}_{s}\left(\mathbf{R}^{d}\right)$ is called the Pilipovic space (of Roumieu type) of order $s \geq 0$ on $\mathbf{R}^{d}$. It follows that $\mathcal{S}_{s}\left(\mathbf{R}^{d}\right)=\mathcal{S}_{s}\left(\mathbf{R}^{d}\right)$ when $s \geq \frac{1}{2}$, but $\emptyset=\mathcal{S}_{s}\left(\mathbf{R}^{d}\right) \neq \mathcal{S}_{s}\left(\mathbf{R}^{d}\right)$ when $0 \leq s<\frac{1}{2}$. 
Remark 1.4. At first glance, the spaces $\boldsymbol{\Sigma}_{s}\left(\mathbf{R}^{d}\right)$ for $s \leq \frac{1}{2}$ and $\mathcal{S}_{s}\left(\mathbf{R}^{d}\right)$ for $s<\frac{1}{2}$ seem to fit well in the family of Gelfand-Shilov spaces, and it is tempting to put $\Sigma_{s}\left(\mathbf{R}^{d}\right)=\boldsymbol{\Sigma}_{s}\left(\mathbf{R}^{d}\right)$ and $\mathcal{S}_{s}\left(\mathbf{R}^{d}\right)=\mathcal{S}_{s}\left(\mathbf{R}^{d}\right)$ for such choices of $s$. Such approach is also justified by (1.3) and the embeddings

$$
\mathcal{S}_{0}\left(\mathbf{R}^{d}\right) \subseteq \boldsymbol{\Sigma}_{s}\left(\mathbf{R}^{d}\right) \subseteq \mathcal{S}_{s}\left(\mathbf{R}^{d}\right) \subseteq \boldsymbol{\Sigma}_{s+\varepsilon}\left(\mathbf{R}^{d}\right), \quad s, \varepsilon>0,
$$

which follow by straight-forward computations.

On the other hand, for such choices of $s$, it is for several reasons appropriate to distinguish between $\boldsymbol{\Sigma}_{s}\left(\mathbf{R}^{d}\right)$ and $\mathcal{S}_{s}\left(\mathbf{R}^{d}\right)$, and the trivial spaces $\Sigma_{s}\left(\mathbf{R}^{d}\right)$ and $\mathcal{S}_{s}\left(\mathbf{R}^{d}\right)$. In fact, the following holds true:

(1) for every $s, t>0$, the spaces $\Sigma_{t}^{s}\left(\mathbf{R}^{d}\right)$ and $\mathcal{S}_{t}^{s}\left(\mathbf{R}^{d}\right)$, and their duals, are invariant under the dilation map $f \mapsto f(\lambda \cdot)$, for every $\lambda \in \mathbf{R} \backslash 0$. On the other hand, $\boldsymbol{\Sigma}_{s}\left(\mathbf{R}^{d}\right)$ when $s \leq \frac{1}{2}$ and $\mathcal{S}_{s}\left(\mathbf{R}^{d}\right)$ when $s<\frac{1}{2}$, are not invariant under such dilations in view of Corollary 6.4 in Section 4 .

(2) Additionally to (1.3) we also have

$$
\begin{aligned}
\Sigma_{t_{1}}^{s_{1}}\left(\mathbf{R}^{d}\right) \subseteq \Sigma_{t_{2}}^{s_{2}}\left(\mathbf{R}^{d}\right) \quad \text { and } \quad \mathcal{S}_{t_{1}}^{s_{1}}\left(\mathbf{R}^{d}\right) \subseteq \mathcal{S}_{t_{2}}^{s_{2}}\left(\mathbf{R}^{d}\right) \\
\quad \text { when } 0<s_{1} \leq s_{2}, 0<t_{1} \leq t_{2} .
\end{aligned}
$$

Now let $\phi(x)=h_{0}(x)=\pi^{-\frac{d}{4}} e^{-\frac{|x|^{2}}{2}}$. Then $\phi \in \Sigma_{\frac{1}{2}}\left(\mathbf{R}^{d}\right)$. On the other hand, we have that $f \in \Sigma_{t}^{s}\left(\mathbf{R}^{d}\right)$, if and only if

$$
|f(x)| \lesssim e^{-r|x|^{\frac{1}{t}}} \text { and }|\widehat{f}(\xi)| \lesssim e^{-r|\xi|^{\frac{1}{s}}}
$$

for every $r>0$. (Cf. e.g. [5, 20].) This implies that $\Sigma_{t}^{s}\left(\mathbf{R}^{d}\right)$ contains no Gauss functions when $s+t>1$, and $s=\frac{1}{2}$ or $t=\frac{1}{2}$. In particular, $\phi \notin \Sigma_{t}^{s}\left(\mathbf{R}^{d}\right)$ for such choices of $s$ and $t$. Consequently, if we set $\Sigma_{\frac{1}{2}}\left(\mathbf{R}^{d}\right)$ equals to $\Sigma_{\frac{1}{2}}\left(\mathbf{R}^{d}\right)$, then (1.8) fails to hold when $s_{1}=t_{1}=\frac{1}{2}, s_{2}+t_{2}>1$, and $s_{2}=s_{1}$ or $t_{2}=t_{1}$.

The dual spaces of $\mathcal{S}_{h, s}\left(\mathbf{R}^{d}\right), \boldsymbol{\Sigma}_{s}\left(\mathbf{R}^{d}\right)$ and $\mathcal{S}_{s}\left(\mathbf{R}^{d}\right)$ are denoted by $\mathcal{S}_{h, s}^{\prime}\left(\mathbf{R}^{d}\right), \boldsymbol{\Sigma}_{s}^{\prime}\left(\mathbf{R}^{d}\right)$ and $\mathcal{S}_{s}^{\prime}\left(\mathbf{R}^{d}\right)$, respectively. In Section 4 it is proved that the supremum norm in (1.6) can be replaced by any $L^{p}$ norm when defining $\boldsymbol{\Sigma}_{s}\left(\mathbf{R}^{d}\right)$ and $\mathcal{S}_{s}\left(\mathbf{R}^{d}\right)$, and their topologies (cf. Proposition 4.1). As a consequence,

$$
\Sigma_{s}^{\prime}\left(\mathbf{R}^{d}\right)=\bigcup_{h>0} \mathcal{S}_{h, s}^{\prime}\left(\mathbf{R}^{d}\right)
$$

when $s>0$ and

$$
\mathcal{S}_{s}^{\prime}\left(\mathbf{R}^{d}\right)=\bigcap_{h>0} \mathcal{S}_{h, s}^{\prime}\left(\mathbf{R}^{d}\right)
$$

when $s \geq 0$, with inductive respective projective limit topologies of $\mathcal{S}_{h, s}^{\prime}\left(\mathbf{R}^{d}\right), h>0$. 
Since Gauss kernels are in background of the most essential parts of our analysis, we restrict our considerations to involve the spaces

$$
\boldsymbol{\Sigma}_{s_{0}+\varepsilon}\left(\mathbf{R}^{d}\right), \quad \mathcal{S}_{s_{0}}\left(\mathbf{R}^{d}\right), \quad \Sigma_{t+\varepsilon}^{s+\varepsilon}\left(\mathbf{R}^{d}\right), \quad \mathcal{S}_{t}^{s}\left(\mathbf{R}^{d}\right), \quad \mathscr{S}\left(\mathbf{R}^{d}\right),
$$

when $s_{0} \geq 0, s, t \geq \frac{1}{2}$ and $\varepsilon>0$, as well as corresponding distribution spaces. For the other situations, other types of analysis are needed in view of (2) in Remark 1.4, and is postponed for the future.

Remark 1.5. We note that in [12,13] it is proved that other elliptic operators can be used to define $\Sigma_{s}$ when $s>\frac{1}{2}$, and $\mathcal{S}_{s}$ when $s \geq$ $\frac{1}{2}$, instead of the harmonic oscillator. Furthermore, in [13] it is also proved that the spaces which correspond to $\Sigma$ are again non-trivial, but in general different from $\Sigma$. The latter fact is also a consequence of Corollary 6.4 in Section 6. In fact, let $f$ be smooth and let $c>0$ be such that $c \neq 1$. Then $f$ fulfills (1.6) for every $h>0$ after $H$ is replaced by $c|x|^{2}-\Delta$, if and only if $x \mapsto f\left(c^{1 / 4} x\right)$ belongs to $\Sigma$. On the other hand, $\Sigma$ is not invariant under dilations in view of Corollary 6.4.

1.3. Modulation spaces. We start by discussing general properties on the involved weight functions. A weight on a Borel set $\Omega \subseteq \mathbf{R}^{d}$ is a positive function $\omega$ such that $\omega$ and $1 / \omega$ belong to $L_{l o c}^{\infty}(\Omega)$.

We recall that if $\omega$ and $v$ are weights on $\mathbf{R}^{d}$, then $\omega$ is called $v$ moderate, or moderate, if

$$
\omega(x+y) \lesssim \omega(x) v(y), \quad x, y \in \mathbf{R}^{d} .
$$

(Cf. [15].) The set of moderate weights on $\mathbf{R}^{d}$ is denoted by $\mathscr{P}_{E}\left(\mathbf{R}^{d}\right)$. If $v$ here above can be chosen as a polynomial, then $\omega$ is called polynomially moderate. The set of polynomially moderate weights on $\mathbf{R}^{d}$ is denoted by $\mathscr{P}\left(\mathbf{R}^{d}\right)$.

We note that $v$ above put limits of the size on $\omega$, since (1.9) implies

$$
v(-x)^{-1} \lesssim \omega(x) \lesssim v(x)
$$

For the larger families of weights in [26], the condition (1.9) is relaxed into

$$
\omega(x) \lesssim \omega(x+y) \lesssim \omega(x) \quad \text { when } \quad R c \leq|x| \leq \frac{c}{|y|}, \quad R \geq 2,
$$

or

$$
\omega(x)^{2} \lesssim \omega(x+y) \omega(x-y) \lesssim \omega(x)^{2}
$$

$$
\text { when } \quad R c \leq|x| \leq \frac{c}{|y|}, \quad R \geq 2, \quad(1.10)^{\prime}
$$

Evidently, if (1.9) holds then (1.10) is true, and if (1.10) holds then $(1.10)^{\prime}$ is true.

We recall that is (1.10) fulfilled for the weights

$$
\omega_{1}(x)=\langle x\rangle^{s} \text { and } \omega_{2}(x)=e^{r|x|^{\frac{1}{t}}}
$$


when $r, s \in \mathbf{R}$ and $t \geq \frac{1}{2}$. On the other hand, $\omega_{2} \notin \mathscr{P}_{E}\left(\mathbf{R}^{d}\right)$ when $t<1$. We also recall that

$$
C^{-1} e^{-c|x|^{2}} \leq \omega(x) \leq C e^{c|x|^{2}},
$$

holds for some positive constants $c$ and $C$ when $\omega$ satisfies (1.10) or $(1.10)^{\prime}$, in view of [26, Proposition 2.13].

Definition 1.6. Let $\omega$ be a weight on $\mathbf{R}^{d}$.

(1) $\mathscr{P}_{Q}\left(\mathbf{R}^{d}\right)$ is the set of all weights $\omega$ on $\mathbf{R}^{d}$ such that $(1.10)^{\prime}$ holds for some positive constants $c$ and $C$;

(2) $\mathscr{P}_{Q}^{0}\left(\mathbf{R}^{d}\right)$ is the set of all weights $\omega \in \mathscr{P}_{Q}\left(\mathbf{R}^{d}\right)$ such that for every $c>0$, there is a constant $C>0$ such that (1.11) holds.

(3) The set $\Omega \subseteq \mathscr{P}_{Q}\left(\mathbf{R}^{d}\right)$ is called an admissible family of weights, if there is a rotation invariant function $0<\omega_{0}(x) \in L_{l o c}^{\infty}\left(\mathbf{R}^{d}\right) \cap$ $L^{1}\left(\mathbf{R}^{d}\right)$ which decreases with $|x|$ and such that

$$
\omega \cdot \omega_{0} \in \Omega \text { and } \omega / \omega_{0} \in \Omega \quad \text { when } \omega \in \Omega .
$$

Example 1.7. Every family in Definition 1.6 are admissible. Moreover, if $\omega_{0} \in \mathscr{P}_{Q}\left(\mathbf{R}^{d}\right)$ and $\Omega$ is a family of admissible weights, then

(1) $\left\{\langle\cdot\rangle^{N} ; N \in \mathbf{Z}\right\}$ is admissible;

(2) $\omega_{0} \cdot \Omega \equiv\left\{\omega_{0} \omega ; \omega \in \Omega\right\}$ is admissible.

In what follows we let $\mathscr{B}$ be a mixed quasi-norm space on $\mathbf{R}^{d}$. This means that for some $p_{1}, \ldots, p_{n} \in(0, \infty]$ and vector spaces

$$
V_{1}, \ldots, V_{n} \subseteq \mathbf{R}^{d} \quad \text { such that } V_{1} \oplus \cdots \oplus V_{n}=\mathbf{R}^{d},
$$

then $\mathscr{B}=\mathscr{B}_{n}$, where $\mathscr{B}_{j}, j=1, \ldots, n$ is inductively defined by

$$
\mathscr{B}_{j}= \begin{cases}L^{p_{1}}\left(V_{1}\right), & j=1 \\ L^{p_{j}}\left(V_{j} ; \mathscr{B}_{j-1}\right), & j=2, \ldots, n .\end{cases}
$$

The minimal exponent $\min \left(p_{1}, \ldots, p_{n}\right)$ is denoted by $\nu_{1}(\mathscr{B})$, and the quasi-norm of $\mathscr{B}$ is given by $\|f\|_{\mathscr{B}} \equiv\left\|F_{n-1}\right\|_{L^{p_{n}}\left(V_{n}\right)}$, where $F_{0}=f$ and

$$
F_{j}\left(x_{j+1}, \ldots, x_{n}\right)=\left\|F_{j-1}\left(\cdot, x_{j+1}, \ldots, x_{n}\right)\right\|_{L^{p_{j}}\left(V_{j}\right)}, \quad j=1, \ldots, n-1 .
$$

Let $p, q \in(0, \infty]$, and $L^{p, q}\left(\mathbf{R}^{2 d}\right)$ be the quasi-Banach spaces, which consist of all measurable $F$ on $\mathbf{R}^{2 d}$ such that

$$
\|F\|_{L^{p, q}} \equiv\left(\int_{\mathbf{R}^{d}}\left(\int_{\mathbf{R}^{d}}|F(x, \xi)|^{p} d x\right)^{q / p} d \xi\right)^{1 / q}<\infty .
$$

(with obvious modifications when $p=\infty$ or $q=\infty$ ). It follows that $L^{p, q}\left(\mathbf{R}^{2 d}\right)$ is a mixed quasi-norm spaces.

The definition of modulation spaces is given in the following.

Definition 1.8. Let $\mathscr{B}$ be a mixed quasi-norm space on $\mathbf{R}^{2 d}, \omega \in$ $\mathscr{P}_{Q}\left(\mathbf{R}^{2 d}\right)$, and let

$$
\phi(x)=\pi^{-\frac{d}{4}} e^{-\frac{|x|^{2}}{2}} .
$$


Then the modulation space $M(\omega, \mathscr{B})$ consists of all $f \in \mathbf{\Sigma}^{\prime}\left(\mathbf{R}^{d}\right)$ such that

$$
\|f\|_{M(\omega, \mathscr{B})} \equiv\left\|V_{\phi} f \omega\right\|_{\mathscr{B}}<\infty .
$$

We also set $M_{(\omega)}^{p, q}\left(\mathbf{R}^{d}\right)=M\left(\omega, L^{p, q}\left(\mathbf{R}^{2 d}\right)\right)$, and $M_{(\omega)}^{p}=M_{(\omega)}^{p, p}$. Furthermore, if in addition $\omega=1$, then we set $M^{p, q}=M_{(\omega)}^{p, q}$ and $M^{p}=M_{(\omega)}^{p}$.

In [26], it is assumed that $f$ in Definition 1.8 should belong to $\mathcal{S}_{\frac{1}{2}}^{\prime}\left(\mathbf{R}^{d}\right)$ instead of the larger class $\boldsymbol{\Sigma}^{\prime}\left(\mathbf{R}^{d}\right)$. This implies that the modulation spaces might be larger in Definition 1.8 compared to [26]. On the other hand, if $\omega$ belongs to $\mathscr{P}_{Q}^{0}\left(\mathbf{R}^{2 d}\right)$ and $f \in M(\omega, \mathscr{B})$, then $f \in \mathcal{S}_{\frac{1}{2}}^{\prime}\left(\mathbf{R}^{d}\right)$ by Theorem 5.2 in Section 5 .

If $\omega$ here above is a moderate weight, then it can be proved that the condition (1.15) is independent of the choice of $\phi \in \Sigma_{1}\left(\mathbf{R}^{d}\right) \backslash 0$, and that different choices of $\phi$ gives rise to equivalent norms (see e. g. Proposition 1.5 in [27]). On the other hand, for general weights in $\mathscr{P}_{Q}\left(\mathbf{R}^{2 d}\right)$, such invariance property seems to not yield.

1.4. Spaces of entire functions and the Bargmann transform. We shall now consider the Bargmann transform which is defined by the formula

$$
\left(\mathfrak{V}_{d} f\right)(z)=\pi^{-\frac{d}{4}} \int_{\mathbf{R}^{d}} \exp \left(-\frac{1}{2}\left(\langle z, z\rangle+|y|^{2}\right)+2^{\frac{1}{2}}\langle z, y\rangle\right) f(y) d y,
$$

when $f \in L^{2}\left(\mathbf{R}^{d}\right)$ (cf. [1]). We note that if $f \in L^{2}\left(\mathbf{R}^{d}\right)$, then the Bargmann transform $\mathfrak{V}_{d} f$ of $f$ is the entire function on $\mathbf{C}^{d}$, given by

or

$$
\left(\mathfrak{V}_{d} f\right)(z)=\int_{\mathbf{R}^{d}} \mathfrak{A}_{d}(z, y) f(y) d y
$$

$$
\left(\mathfrak{V}_{d} f\right)(z)=\left\langle f, \mathfrak{A}_{d}(z, \cdot)\right\rangle,
$$

where the Bargmann kernel $\mathfrak{A}_{d}$ is given by

$$
\mathfrak{A}_{d}(z, y)=\pi^{-\frac{d}{4}} \exp \left(-\frac{1}{2}\left(\langle z, z\rangle+|y|^{2}\right)+2^{\frac{1}{2}}\langle z, y\rangle\right) .
$$

Here

$$
\begin{gathered}
\langle z, w\rangle=\sum_{j=1}^{d} z_{j} w_{j}, \quad \text { when } z=\left(z_{1}, \ldots, z_{d}\right) \in \mathbf{C}^{d} \\
\quad \text { and } \quad w=\left(w_{1}, \ldots, w_{d}\right) \in \mathbf{C}^{d}
\end{gathered}
$$

and otherwise $\langle\cdot, \cdot\rangle$ denotes the duality between test function spaces and their corresponding duals. We note that the right-hand side in (1.16) makes sense when $f \in \mathcal{S}_{\frac{1}{2}}^{\prime}\left(\mathbf{R}^{d}\right)$ and defines an element in $A\left(\mathbf{C}^{d}\right)$, since $y \mapsto \mathfrak{A}_{d}(z, y)$ can be interpreted as an element in $\mathcal{S}_{\frac{1}{2}}\left(\mathbf{R}^{d}\right)$ with values in $A\left(\mathbf{C}^{d}\right)$. Here and in what follows, $A(\Omega)$ denotes the set of analytic functions on the open set $\Omega \subseteq \mathbf{C}^{d}$. 
It was proved in [1] that $f \mapsto \mathfrak{V}_{d} f$ is a bijective and isometric map from $L^{2}\left(\mathbf{R}^{d}\right)$ to the Hilbert space $A^{2}\left(\mathbf{C}^{d}\right) \equiv B^{2}\left(\mathbf{C}^{d}\right) \cap A\left(\mathbf{C}^{d}\right)$, where $B^{2}\left(\mathbf{C}^{d}\right)$ consists of all measurable functions $F$ on $\mathbf{C}^{d}$ such that

$$
\|F\|_{B^{2}} \equiv\left(\int_{\mathbf{C}^{d}}|F(z)|^{2} d \mu(z)\right)^{\frac{1}{2}}<\infty .
$$

Here $d \mu(z)=\pi^{-d} e^{-|z|^{2}} d \lambda(z)$, where $d \lambda(z)$ is the Lebesgue measure on $\mathbf{C}^{d}$. We recall that $A^{2}\left(\mathbf{C}^{d}\right)$ and $B^{2}\left(\mathbf{C}^{d}\right)$ are Hilbert spaces, where the scalar product are given by

$$
(F, G)_{B^{2}} \equiv \int_{\mathbf{C}^{d}} F(z) \overline{G(z)} d \mu(z), \quad F, G \in B^{2}\left(\mathbf{C}^{d}\right) .
$$

If $F, G \in A^{2}\left(\mathbf{C}^{d}\right)$, then we set $\|F\|_{A^{2}}=\|F\|_{B^{2}}$ and $(F, G)_{A^{2}}=(F, G)_{B^{2}}$.

Furthermore, Bargmann proved that there is a convenient reproducing. formula on $A^{2}\left(\mathbf{C}^{d}\right)$. More precisely, let

$$
\left(\Pi_{A} F\right)(z) \equiv \int_{\mathbf{C}^{d}} F(w) e^{(z, w)} d \mu(w)
$$

when $F e^{R|\cdot|-|\cdot|^{2}} \in L^{1}\left(\mathbf{C}^{d}\right)$, for every $R \geq 0$. Here

$$
\begin{gathered}
(z, w)=\sum_{j=1}^{d} z_{j} \overline{w_{j}}, \quad \text { when } z=\left(z_{1}, \ldots, z_{d}\right) \in \mathbf{C}^{d} \\
\quad \text { and } \quad w=\left(w_{1}, \ldots, w_{d}\right) \in \mathbf{C}^{d}
\end{gathered}
$$

is the scalar product of $z \in \mathbf{C}^{d}$ and $w \in \mathbf{C}^{d}$. Then it is proved in [1,2] that $\Pi_{A} F=F$ when $F \in A^{2}\left(\mathbf{C}^{d}\right)$.

In [1] it is also proved that

$$
\mathfrak{V}_{d} h_{\alpha}=e_{\alpha}, \quad \text { where } \quad e_{\alpha}(z) \equiv \frac{z^{\alpha}}{\sqrt{\alpha !}}, \quad z \in \mathbf{C}^{d} .
$$

In particular, the Bargmann transform maps the orthonormal basis $\left\{h_{\alpha}\right\}_{\alpha \in \mathbf{N}^{d}}$ in $L^{2}\left(\mathbf{R}^{d}\right)$ bijectively into the orthonormal basis $\left\{e_{\alpha}\right\}_{\alpha \in \mathbf{N}^{d}}$ of monomials in $A^{2}\left(\mathbf{C}^{d}\right)$. Furthermore, if $f, g \in L^{2}\left(\mathbf{R}^{d}\right)$ and $F, G \in$ $A^{2}\left(\mathbf{C}^{d}\right)$ are given by

$$
\begin{aligned}
& f=\sum_{\alpha \in \mathbf{N}^{d}} a_{\alpha} h_{\alpha}, \quad g=\sum_{\alpha \in \mathbf{N}^{d}} b_{\alpha} h_{\alpha} \\
& F=\sum_{\alpha \in \mathbf{N}^{d}} a_{\alpha} e_{\alpha}, \quad G=\sum_{\alpha \in \mathbf{N}^{d}} b_{\alpha} e_{\alpha}
\end{aligned}
$$

then $F=\mathfrak{V}_{d} f, G=\mathfrak{V}_{d} g$ and

$$
(f, g)_{L^{2}}=(F, G)_{A^{2}}=\sum_{\alpha \in \mathbf{N}^{d}} a_{\alpha} \overline{b_{\alpha}}
$$

Here and in what follows, $(\cdot, \cdot)_{L^{2}\left(\mathbf{R}^{d}\right)}$ and $(\cdot, \cdot)_{A^{2}\left(\mathbf{C}^{d}\right)}$ denote the scalar products in $L^{2}\left(\mathbf{R}^{d}\right)$ and $A^{2}\left(\mathbf{C}^{d}\right)$, respectively. 
Next we recall the link between the Bargmann transform and the short-time Fourier transform $f \mapsto V_{\phi} f$, when $\phi$ is given by (1.14). Let $S$ be the dilation operator given by

$$
(S F)(x, \xi)=F\left(2^{-\frac{1}{2}} x,-2^{-\frac{1}{2}} \xi\right),
$$

when $F \in L_{l o c}^{1}\left(\mathbf{R}^{2 d}\right)$. Then it follows by straight-forward computations that

$$
\begin{array}{r}
\left(\mathfrak{V}_{d} f\right)(z)=\left(\mathfrak{V}_{d} f\right)(x+i \xi)=(2 \pi)^{\frac{d}{2}} e^{\frac{1}{2}\left(|x|^{2}+|\xi|^{2}\right)} e^{-i\langle x, \xi\rangle} V_{\phi} f\left(2^{\frac{1}{2}} x,-2^{\frac{1}{2}} \xi\right) \\
=(2 \pi)^{\frac{d}{2}} e^{\frac{1}{2}\left(|x|^{2}+|\xi|^{2}\right)} e^{-i\langle x, \xi\rangle}\left(S^{-1}\left(V_{\phi} f\right)\right)(x, \xi),
\end{array}
$$

or equivalently,

$$
\begin{array}{r}
V_{\phi} f(x, \xi)=(2 \pi)^{-\frac{d}{2}} e^{-\frac{1}{4}\left(|x|^{2}+|\xi|^{2}\right)} e^{-i\langle x, \xi\rangle / 2}\left(\mathfrak{V}_{d} f\right)\left(2^{-\frac{1}{2}} x,-2^{-\frac{1}{2}} \xi\right) . \\
=(2 \pi)^{-\frac{d}{2}} e^{-i\langle x, \xi\rangle / 2} S\left(e^{-\frac{|\cdot|^{2}}{2}}\left(\mathfrak{V}_{d} f\right)\right)(x, \xi) .
\end{array}
$$

We observe that (1.24) and (1.25) can be formulated into

$$
\mathfrak{V}_{d}=U_{\mathfrak{V}} \circ V_{\phi}, \quad \text { and } \quad U_{\mathfrak{V}}^{-1} \circ \mathfrak{V}_{d}=V_{\phi},
$$

where $U_{\mathfrak{V}}$ is the linear, continuous and bijective operator on $\mathscr{D}^{\prime}\left(\mathbf{R}^{2 d}\right) \simeq$ $\mathscr{D}^{\prime}\left(\mathbf{C}^{d}\right)$, given by

$$
\left(U_{\mathfrak{V}} F\right)(x, \xi)=(2 \pi)^{\frac{d}{2}} e^{\frac{1}{2}\left(|x|^{2}+|\xi|^{2}\right)} e^{-i\langle x, \xi\rangle} F\left(2^{\frac{1}{2}} x,-2^{\frac{1}{2}} \xi\right) .
$$

Definition 1.9. Let $\omega$ be a weight on $\mathbf{R}^{2 d}, \mathscr{B}$ be a mixed quasi-norm space on $\mathbf{R}^{2 d} \simeq \mathbf{C}^{d}$, and let $r>0$ be such that $r \leq \nu_{1}(\mathscr{B})$.

(1) The space $B(\omega, \mathscr{B})$ is the modified weighted $\mathscr{B}$-space which consists of all $F \in L_{l o c}^{r}\left(\mathbf{R}^{2 d}\right)=L_{l o c}^{r}\left(\mathbf{C}^{d}\right)$ such that

$$
\|F\|_{B(\omega, \mathscr{B})} \equiv\left\|\left(U_{\mathfrak{V}}^{-1} F\right) \omega\right\|_{\mathscr{B}}<\infty \text {. }
$$

Here $U_{\mathfrak{V}}$ is given by (1.26);

(2) The space $A(\omega, \mathscr{B})$ consists of all $F \in A\left(\mathbf{C}^{d}\right) \cap B(\omega, \mathscr{B})$ with topology inherited from $B(\omega, \mathscr{B})$.

We note that the spaces in Definition 1.9 are normed spaces when $\nu_{1}(\mathscr{B}) \geq 1$.

For convenience we set $\|F\|_{B(\omega, \mathscr{B})}=\infty$, when $F \notin B(\omega, \mathscr{B})$ is measurable, and $\|F\|_{A(\omega, \mathscr{B})}=\infty$, when $F \in A\left(\mathbf{C}^{d}\right) \backslash B(\omega, \mathscr{B})$. We also set

$$
A_{(\omega)}^{p, q}\left(\mathbf{C}^{d}\right)=A\left(\omega, L^{p, q}\left(\mathbf{C}^{d}\right)\right) \quad \text { and } \quad A_{(\omega)}^{p}=A_{(\omega)}^{p, p} .
$$

The following result follows from Theorems 3.4 and 5.1 in [26], and justify the definition of the spaces in Definition 1.9.

Proposition 1.10. Let $\omega \in \mathscr{P}_{Q}^{0}\left(\mathbf{R}^{2 d}\right)$, $\mathscr{B}$ be a mixed quasi-norm space on $\mathbf{R}^{2 d}$ and let $\phi$ be as in (1.14). Then the following is true:

(1) the map $\mathfrak{V}_{d}$ is an isometric bijection from $M(\omega, \mathscr{B})$ to $A(\omega, \mathscr{B})$; 
(2) the map $\Pi_{A}$ is a continuos projection from $B(\omega, \mathscr{B})$ to $A(\omega, \mathscr{B})$.

The case $\omega=1$ and $\mathscr{B}=L^{2}$ was proved already in [1]. In Section 3 we extend Proposition 1.10(1) in such way that the only assumption $\omega \in \mathscr{P}_{Q}^{0}\left(\mathbf{R}^{2 d}\right)$ is that it should be a weight on $\mathbf{R}^{2 d}$.

Let $D_{r}\left(z_{0}\right)$ be the polydisc

$$
\left\{z=\left(z_{1}, \ldots, z_{d}\right) \in \mathbf{C}^{d} ;\left|z_{j}-z_{0, j}\right|<r_{j}, j=1, \ldots, d\right\},
$$

with respect to

$$
z_{0}=\left(z_{0,1}, \ldots, z_{0, d}\right) \in \mathbf{C}^{d}, \quad \text { and } \quad r=\left(r_{1}, \ldots, r_{d}\right) \in[0, \infty)^{d},
$$

and let $A_{d}\left\{z_{0}\right\}$ be the set of all functions which are analytic at $z_{0}$. Then

$$
A\left(\mathbf{C}^{d}\right)=\bigcap_{r \in[0, \infty)^{d}} A\left(D_{r}(z)\right), \quad A_{d}\left\{z_{0}\right\}=\bigcup_{r \in[0, \infty)^{d}} A\left(D_{r}\left(z_{0}\right)\right)
$$

The following result is a straight-forward consequence of Theorem 3.2 in [26].

Proposition 1.11. Let $p_{1}, p_{2} \in(0, \infty], s, t \geq \frac{1}{2}, r, r_{1}, r_{2} \in \mathbf{R}$ be such that $r_{2}<r_{1}$,

$$
\vartheta_{r, s, t}(z)=e^{r\left(|x|^{\frac{1}{t}}+|\xi|^{\frac{1}{s}}\right)}, \quad z=x+i \xi, x, \xi \in \mathbf{R}^{d},
$$

and let $\sigma_{r}(z)=\langle z\rangle^{r}$. Then

$$
\begin{gathered}
A_{\left(\vartheta_{r_{1}, s, t}\right)}^{p_{1}}\left(\mathbf{C}^{d}\right) \subseteq A_{\left(\vartheta_{r_{2}, s, t}\right)}^{p_{2}}\left(\mathbf{C}^{d}\right), \\
A_{\left(\vartheta_{r, s, t} \sigma_{N}\right)}^{p_{1}}\left(\mathbf{C}^{d}\right) \subseteq A_{\left(\vartheta_{r, s, t}\right)}^{p_{2}}\left(\mathbf{C}^{d}\right) \subseteq A_{\left(\vartheta_{r, s, t} \sigma_{-N}\right)}^{p_{1}}\left(\mathbf{C}^{d}\right), \\
\|F\|_{A_{\left(\vartheta_{r_{2}, s, t}\right)}^{p_{2}}} \lesssim\|F\|_{A_{\left(\vartheta_{r_{1}, s, t}\right)}^{p_{1}}}
\end{gathered}
$$

and

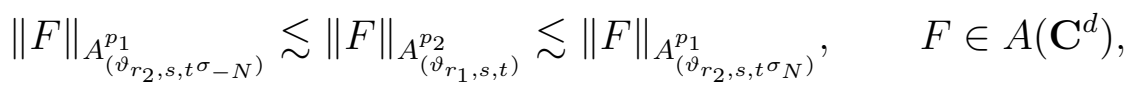

provided $N \geq 0$ is chosen large enough.

Proof. Let $\Omega$ be the set of all weights $z \mapsto \omega_{r_{2}, s, t}(z)\langle z\rangle^{h}, h \in \mathbf{R}$. Then $\Omega$ is an admissible family of weights. By Theorem 3.2 in [26] we get

$$
\|F\|_{A_{\left(\omega_{\left.s, t, r_{2}\right)}\right.}^{p_{2}}} \lesssim\|F\|_{A_{\left(\omega_{s, t, r_{2}} \sigma_{r}\right)}^{p_{1}}}, \quad F \in A\left(\mathbf{C}^{d}\right)
$$

provided $r \geq 0$ is chosen large enough. Since $\omega_{s, t, r_{2}} \sigma_{N} \lesssim \omega_{s, t, r_{1}}$, we get

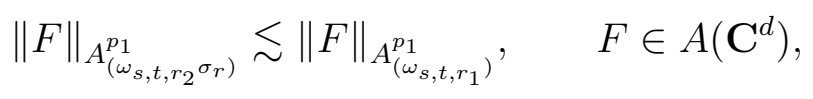

and the first inclusion and the first inequality in the assertion follow from these estimates. The other parts follow by similar arguments and are left for the reader. 
Remark 1.12. Later on we consider consider spaces of entire functions of the forms

$$
\bigcup_{r>0} A_{\left(\vartheta_{r}\right)}^{\infty}\left(\mathbf{C}^{d}\right)=\left\{F \in A\left(\mathbf{C}^{d}\right) ;|F(z)| \lesssim \omega_{r}(z) \text { for some } r>0\right\}
$$

and

$$
\bigcap_{r>0} A_{\left(\vartheta_{r}\right)}^{\infty}\left(\mathbf{C}^{d}\right)=\left\{F \in A\left(\mathbf{C}^{d}\right) ;|F(z)| \lesssim \omega_{r}(z) \text { for every } r>0\right\}
$$

for a suitable family $\left\{\omega_{r}\right\}_{r>0}$ of weights on $\mathbf{C}^{d}$. Here $\vartheta_{r}(z)=e^{-\frac{|z|^{2}}{2}} \omega_{r}(z)$. We let the topologies of the sets in (1.28) and (1.29) be the inductive limit and projective limit topologies of $A_{\left(\vartheta_{r}\right)}^{\infty}\left(\mathbf{C}^{d}\right)$, respectively.

\section{HeRmite AND POWER SERIES EXPANSIONS, AND THE BARGMANN TRANSFORM}

In this section we consider topological vector spaces of Hermite series expansions and link them to topological vector spaces of power series expansions through the Bargmann transform. In the case when a Hilbert space of power series expansions is equal to $A_{(\omega)}^{2}\left(\mathbf{C}^{d}\right)$ for suitable $\omega$, then we deduce explicit formulas between involved weights for the set of Hermite series expansions and the weight $\omega$.

\subsection{Topological vector spaces of Hermite series and power se-} ries expansions. The spaces of series expansions depend on parameters in the extended sets

$$
\mathbf{R}_{b}=\mathbf{R}_{+} \bigcup\left\{b_{\sigma} ; \sigma>0\right\} \quad \text { and } \overline{\mathbf{R}_{b}}=\mathbf{R}_{b} \bigcup\{0\},
$$

of positive real numbers. For conveniency we also set $b_{\infty}=\frac{1}{2}$. Beside the usual ordering in $\mathbf{R}$, the elements $b_{\sigma}$ in $\mathbf{R}_{b}$ and $\overline{\mathbf{R}_{b}}$ are ordered by the relations $x_{1}<b_{\sigma_{1}}<b_{\sigma_{2}}<x_{2}$, when $\sigma_{1}<\sigma_{2}$, and $x_{1}<\frac{1}{2}$ and $x_{2} \geq \frac{1}{2}$ are real.

Definition 2.1. Let $p \in(0, \infty], r, t \in \mathbf{R}_{+}, s \in \mathbf{R}_{b}, \vartheta$ be a weight on $\mathbf{N}^{d}$, and let

$$
\vartheta_{r, s}(\alpha) \equiv \begin{cases}e^{r|\alpha| \frac{1}{2 s}}, & \text { when } \quad s \in \mathbf{R}_{+}, \\ r^{|\alpha|}(\alpha !)^{\frac{1}{2 \sigma}}, & \text { when } s=b_{\sigma}, \quad \alpha \in \mathbf{N}^{d} .\end{cases}
$$

Then

(1) $\ell_{0}^{\prime}\left(\mathbf{N}^{d}\right)$ is the set of all sequences $\left\{c_{\alpha}\right\}_{\alpha \in \mathbf{N}^{d}} \subseteq \mathbf{C}$ on $\mathbf{N}^{d}$;

(2) $\ell_{0,0}\left(\mathbf{N}^{d}\right) \equiv\{0\}$, and $\ell_{0}\left(\mathbf{N}^{d}\right)$ is the set of all sequences $\left\{c_{\alpha}\right\}_{\alpha \in \mathbf{N}^{d}} \subseteq$ $\mathrm{C}$ such that $c_{\alpha} \neq 0$ for at most finite numbers of $\alpha$;

(3) $\ell_{[\vartheta]}^{p}\left(\mathbf{N}^{d}\right)$ is the quasi-Banach space which consists of all sequences $\left\{c_{\alpha}\right\}_{\alpha \in \mathbf{N}^{d}} \subseteq \mathbf{C}$ such that

$$
\left\|\left\{c_{\alpha}\right\}_{\alpha \in \mathbf{N}^{d}}\right\|_{\ell_{[\vartheta]}^{p}} \equiv\left\|\left\{c_{\alpha} \vartheta(\alpha)\right\}_{\alpha \in \mathbf{N}^{d}}\right\|_{\ell^{p}}
$$


is finite;

(4) $\ell_{0, s}\left(\mathbf{N}^{d}\right) \equiv \bigcap_{r>0} \ell_{\left[\vartheta_{r, s}\right]}^{p}\left(\mathbf{N}^{d}\right)$ and $\ell_{s}\left(\mathbf{N}^{d}\right) \equiv \bigcup_{r>0} \ell_{\left[\vartheta_{r, s}\right]}^{p}\left(\mathbf{N}^{d}\right)$, with projective respective inductive limit topologies of $\ell_{\left[\vartheta_{r, s}\right]}^{p}\left(\mathbf{N}^{d}\right)$ with respect to $r>0$;

(5) $\ell_{0, s}^{\prime}\left(\mathbf{N}^{d}\right) \equiv \bigcup_{r>0} \ell_{\left[1 / \vartheta_{r, s}\right]}^{p}\left(\mathbf{N}^{d}\right)$ and $\ell_{s}^{\prime}\left(\mathbf{N}^{d}\right) \equiv \bigcap_{r>0} \ell_{\left[1 / \vartheta_{r, s}\right]}^{p}\left(\mathbf{N}^{d}\right)$, with inductive respective projective limit topologies of $\ell_{\left[1 / \vartheta_{r, s}\right]}^{p}\left(\mathbf{N}^{d}\right)$ with respect to $r>0$.

Let $p \in(0, \infty]$, and let $\Omega_{N}$ be the set of all $\alpha \in \mathbf{N}^{d}$ such that $|\alpha| \leq N$. Then the topology of $\ell_{0}\left(\mathbf{N}^{d}\right)$ is defined by the inductive limit topology of the sets

$$
\left\{\left\{c_{\alpha}\right\}_{\alpha \in \mathbf{N}^{d}} \in \ell_{0}^{\prime}\left(\mathbf{N}^{d}\right) ; c_{\alpha}=0 \text { when } \alpha \neq \Omega_{N}\right\}
$$

with respect to $N \geq 0$, and whose topology is given through the quasinorms

$$
\left\{c_{\alpha}\right\}_{\alpha \in \mathbf{N}^{d}} \mapsto\left\|\left\{c_{\alpha}\right\}_{|\alpha| \leq N}\right\|_{\ell^{p}\left(\Omega_{N}\right)},
$$

Since any two such quasi-norms on a finite-dimensional vector space are equivalent, it follows that these topologies are independent of $p$. Furthermore, $\ell_{0}^{\prime}\left(\mathbf{N}^{d}\right)$ is a Fréchet space and independent of $p$ when the topology is defined by the quasi-semi-norms (2.2).

Next we introduce spaces of formal Hermite series expansions

$$
f=\sum_{\alpha \in \mathbf{N}^{d}} c_{\alpha} h_{\alpha}, \quad\left\{c_{\alpha}\right\}_{\alpha \in \mathbf{N}^{d}} \in \ell_{0}^{\prime}\left(\mathbf{N}^{d}\right)
$$

and spaces of formal power series expansions

$$
F=\sum_{\alpha \in \mathbf{N}^{d}} c_{\alpha} e_{\alpha}, \quad\left\{c_{\alpha}\right\}_{\alpha \in \mathbf{N}^{d}} \in \ell_{0}^{\prime}\left(\mathbf{N}^{d}\right),
$$

which correspond to

$$
\ell_{0, s}\left(\mathbf{N}^{d}\right), \quad \ell_{s}\left(\mathbf{N}^{d}\right), \quad \ell_{[\vartheta]}^{p}\left(\mathbf{N}^{d}\right), \quad \ell_{s}^{\prime}\left(\mathbf{N}^{d}\right) \quad \text { and } \quad \ell_{0, s}^{\prime}\left(\mathbf{N}^{d}\right) .
$$

Here $e_{\alpha}$ are given by (1.20). For that reason we consider the mappings

$$
T_{1}:\left\{c_{\alpha}\right\}_{\alpha \in \mathbf{N}^{d}} \mapsto \sum_{\alpha \in \mathbf{N}^{d}} c_{\alpha} h_{\alpha}
$$

and

$$
T_{2}:\left\{c_{\alpha}\right\}_{\alpha \in \mathbf{N}^{d}} \mapsto \sum_{\alpha \in \mathbf{N}^{d}} c_{\alpha} e_{\alpha}
$$

between sequences and series expansions.

Definition 2.2. Let $p \in(0, \infty], s \in \overline{\mathbf{R}_{b}}, \vartheta$ be a weight on $\mathbf{N}^{d}$, and let $e_{\alpha}$ be given by (1.20). 
- the images of $T_{1}$ in (2.6) of the spaces in (2.5) are denoted by

$$
\mathcal{H}_{0, s}\left(\mathbf{R}^{d}\right), \quad \mathcal{H}_{s}\left(\mathbf{R}^{d}\right), \quad \mathcal{H}_{[\vartheta]}^{p}\left(\mathbf{R}^{d}\right), \quad \mathcal{H}_{s}^{\prime}\left(\mathbf{R}^{d}\right) \quad \text { and } \quad \mathcal{H}_{0, s}^{\prime}\left(\mathbf{R}^{d}\right)
$$

respectively. Furthermore, the topologies of the spaces in (2.8) are inherited from corresponding spaces in (2.5).

- the images of $T_{2}$ in (2.7) of the spaces in (2.5) are denoted by

$$
\mathcal{A}_{0, s}\left(\mathbf{C}^{d}\right), \quad \mathcal{A}_{s}\left(\mathbf{C}^{d}\right), \quad \mathcal{A}_{[\vartheta]}^{p}\left(\mathbf{C}^{d}\right), \quad \mathcal{A}_{s}^{\prime}\left(\mathbf{C}^{d}\right) \quad \text { and } \quad \mathcal{A}_{0, s}^{\prime}\left(\mathbf{C}^{d}\right)
$$

respectively. Furthermore, the topologies of the spaces in (2.9) are inherited from corresponding spaces in (2.5).

- the quasi-norms $\|f\|_{\mathcal{H}_{[\vartheta]}^{p}}$ and $\|F\|_{\mathcal{A}_{[\vartheta]}^{p}}$ of $f \in \mathcal{H}_{0}^{\prime}\left(\mathbf{R}^{d}\right)$ and $F \in$ $\mathcal{A}_{0}^{\prime}\left(\mathbf{C}^{d}\right)$, respectively, are given by $\left\|\left\{c_{\alpha}\right\}_{\alpha \in \mathbf{N}^{d}}\right\|_{\ell_{[\vartheta]}^{p}}$, when $f$ and $F$ are given by (2.3) and (2.4), respectively.

For any $f \in \mathcal{H}_{0}^{\prime}\left(\mathbf{R}^{d}\right)$ with expansion (2.3) , the coefficients $c_{\alpha}$ are still called the Hermite coefficients for $f$ and are usually denoted by $c_{\alpha}(f)$. Hence (2.3) takes the form

$$
f=\sum_{\alpha} c_{\alpha}(f) h_{\alpha}, \quad c_{\alpha}(f)=\left(f, h_{\alpha}\right)_{L^{2}}
$$

Evidently, in (2.5), (2.8) and (2.9), the largest spaces are

$$
\ell_{0}^{\prime}\left(\mathbf{N}^{d}\right), \quad \mathcal{H}_{0}^{\prime}\left(\mathbf{R}^{d}\right) \quad \text { and } \quad \mathcal{A}_{0}^{\prime}\left(\mathbf{C}^{d}\right)
$$

respectively, and the smallest non-trivial spaces are

$$
\ell_{0}\left(\mathbf{N}^{d}\right), \quad \mathcal{H}_{0}\left(\mathbf{R}^{d}\right) \quad \text { and } \quad \mathcal{A}_{0}\left(\mathbf{C}^{d}\right)
$$

respectively. By the definitions it also follows that the following holds true.

Proposition 2.3. Let $s \in \overline{\mathbf{R}_{b}}, p \in(0, \infty]$ and let $\vartheta$ be a weight on $\mathbf{N}^{d}$. Then the following is true:

(1) the map $T_{1}$ in (2.6) is a homeomorphism from $\ell_{0, s}\left(\mathbf{N}^{d}\right)$ to $\mathcal{H}_{0, s}\left(\mathbf{R}^{d}\right)$, from $\ell_{s}\left(\mathbf{N}^{d}\right)$ to $\mathcal{H}_{s}\left(\mathbf{R}^{d}\right)$, from $\ell_{s}^{\prime}\left(\mathbf{N}^{d}\right)$ to $\mathcal{H}_{s}^{\prime}\left(\mathbf{R}^{d}\right)$, and from $\ell_{0, s}^{\prime}\left(\mathbf{N}^{d}\right)$ to $\mathcal{H}_{0, s}^{\prime}\left(\mathbf{R}^{d}\right)$;

(2) the map $T_{2}$ in (2.7) is a homeomorphism from $\ell_{0, s}\left(\mathbf{N}^{d}\right)$ to $\mathcal{A}_{0, s}\left(\mathbf{C}^{d}\right)$, from $\ell_{s}\left(\mathbf{N}^{d}\right)$ to $\mathcal{A}_{s}\left(\mathbf{C}^{d}\right)$, from $\ell_{s}^{\prime}\left(\mathbf{N}^{d}\right)$ to $\mathcal{A}_{s}^{\prime}\left(\mathbf{C}^{d}\right)$, and from $\ell_{0, s}^{\prime}\left(\mathbf{N}^{d}\right)$ to $\mathcal{A}_{0, s}^{\prime}\left(\mathbf{C}^{d}\right)$;

(3) the mappings $T_{1}$ and $T_{2}$ in (2.6) and (2.7) are isometric bijections from $\ell_{[\vartheta]}^{p}\left(\mathbf{N}^{d}\right)$ to $\mathcal{H}_{[\vartheta]}^{p}\left(\mathbf{R}^{d}\right)$, and from $\ell_{[\vartheta]}^{p}\left(\mathbf{N}^{d}\right)$ to $\mathcal{A}_{[\vartheta]}^{p}\left(\mathbf{C}^{d}\right)$, respectively. 
In the sequel we let the conjugate exponent $p^{\prime}$ of $p \in(0, \infty]$ be defined by

$$
p^{\prime}=\left\{\begin{array}{cl}
1, & p=\infty \\
\frac{p}{p-1}, & 1<p<\infty \\
\infty, & 0<p \leq 1
\end{array}\right.
$$

Remark 2.4. Let $s \in \overline{\mathbf{R}_{b}}$ and $p \in(0, \infty]$ be the same as in Definitions 2.1 and 2.2. By straight-forward computations it follows that the following is true:

- $\ell_{0, s}\left(\mathbf{N}^{d}\right), \ell_{s}\left(\mathbf{N}^{d}\right), \ell_{0, s}^{\prime}\left(\mathbf{N}^{d}\right), \ell_{s}^{\prime}\left(\mathbf{N}^{d}\right)$ and their topologies are independent of $p \in(0, \infty]$;

- the duals of $\ell_{0}\left(\mathbf{N}^{d}\right), \ell_{0, s}\left(\mathbf{N}^{d}\right)$ and $\ell_{s}\left(\mathbf{N}^{d}\right)$ are equal to $\ell_{0}^{\prime}\left(\mathbf{N}^{d}\right)$, $\ell_{0, s}^{\prime}\left(\mathbf{N}^{d}\right)$ and $\ell_{s}^{\prime}\left(\mathbf{N}^{d}\right)$, respectively (also in topological sense), through unique extensions of the $\ell^{2}$-form on $\ell_{0}\left(\mathbf{N}^{d}\right) \times \ell_{0}\left(\mathbf{N}^{d}\right)$;

- if in addition $p \in[1, \infty)$, then the dual of $\ell_{[\vartheta]}^{p}\left(\mathbf{N}^{d}\right)$ can be identified by $\ell_{[1 / \vartheta]}^{p^{\prime}}\left(\mathbf{N}^{d}\right)$ through a unique extension the $\ell^{2}$-form on $\ell_{0}\left(\mathbf{N}^{d}\right) \times \ell_{0}\left(\mathbf{N}^{d}\right)$.

By Proposition 2.3, the same holds true if the spaces in (2.5) and the $\ell^{2}$ form are replaced by the spaces in (2.8) and the $L^{2}$-form, respectively, or replaced by the spaces in (2.9) and the $A^{2}$-form, respectively.

In particular, if $f$ and $F$ are given by (2.3) and (2.4), and

$$
f_{0}=\sum_{\alpha \in \mathbf{N}^{d}} c_{0, \alpha} h_{\alpha} \in \mathcal{H}_{0}\left(\mathbf{R}^{d}\right) \quad \text { and } \quad F_{0}=\sum_{\alpha \in \mathbf{N}^{d}} c_{0, \alpha} e_{\alpha} \in \mathcal{A}_{0}\left(\mathbf{C}^{d}\right),
$$

for some $\left\{c_{0, \alpha}\right\}_{\alpha \in \mathbf{N}^{d}} \in \ell_{0}\left(\mathbf{N}^{d}\right)$, then

$$
\left(\left\{c_{0, \alpha}\right\}_{\alpha \in \mathbf{N}^{d}},\left\{c_{\alpha}\right\}_{\alpha \in \mathbf{N}^{d}}\right)_{\ell^{2}}=\left(f_{0}, f\right)_{L^{2}}=\left(F_{0}, F\right)_{A^{2}}=\sum_{\alpha \in \mathbf{N}^{d}} c_{0, \alpha} \overline{c_{\alpha}} .
$$

The Bargmann transform $\mathfrak{V}_{d} f$ of $f \in \mathcal{H}_{0}^{\prime}\left(\mathbf{R}^{d}\right)$ is defined as the righthand side of (2.4) when $f$ is given by (2.3). That is,

$$
\mathfrak{V}_{d} f=\sum_{\alpha \in \mathbf{N}^{d}} c_{\alpha} e_{\alpha} \in \mathcal{A}_{0}^{\prime}\left(\mathbf{C}^{d}\right) \quad \text { when } \quad f=\sum_{\alpha \in \mathbf{N}^{d}} c_{\alpha} h_{\alpha} \in \mathcal{H}_{0}^{\prime}\left(\mathbf{R}^{d}\right)
$$

In particular, Proposition 2.3 shows that the mappings $T_{1}, T_{2}$ and $\mathfrak{V}_{d}$ induce homeomorphisms, still denoted by $T_{1}, T_{2}$ and $\mathfrak{V}_{d}$, respectively, between Gelfand-tripples of suitable spaces in (2.2), (2.8) and (2.9). More precisely, the mappings

$$
\begin{aligned}
& \left(\ell_{s}\left(\mathbf{N}^{d}\right), \ell^{2}\left(\mathbf{N}^{d}\right), \ell_{s}^{\prime}\left(\mathbf{N}^{d}\right)\right) \quad \stackrel{T_{1}}{\rightarrow}\left(\mathcal{H}_{s}\left(\mathbf{R}^{d}\right), L^{2}\left(\mathbf{R}^{d}\right), \mathcal{H}_{s}^{\prime}\left(\mathbf{R}^{d}\right)\right) \\
& T_{2} \searrow \quad \downarrow \mathfrak{V}_{d} \\
& \left(\mathcal{A}_{s}\left(\mathbf{C}^{d}\right), A^{2}\left(\mathbf{C}^{d}\right), \mathcal{A}_{s}^{\prime}\left(\mathbf{C}^{d}\right)\right)
\end{aligned}
$$


are homeomorphisms for every $s \in \overline{\mathbf{R}_{b}}$.

$$
\begin{aligned}
& \left(\ell_{0, s}\left(\mathbf{N}^{d}\right), \ell^{2}\left(\mathbf{N}^{d}\right), \ell_{0, s}^{\prime}\left(\mathbf{N}^{d}\right)\right) \quad \stackrel{T_{1}}{\rightarrow}\left(\mathcal{H}_{0, s}\left(\mathbf{R}^{d}\right), L^{2}\left(\mathbf{R}^{d}\right), \mathcal{H}_{0, s}^{\prime}\left(\mathbf{R}^{d}\right)\right) \\
& T_{2} \searrow \quad \downarrow \mathfrak{V}_{d} \\
& \left(\mathcal{A}_{0, s}\left(\mathbf{C}^{d}\right), A^{2}\left(\mathbf{C}^{d}\right), \mathcal{A}_{0, s}^{\prime}\left(\mathbf{C}^{d}\right)\right)
\end{aligned}
$$

are homeomorphisms for every $s \in \mathbf{R}_{b}$, and

$$
\begin{aligned}
\left(\ell_{[\vartheta]}^{p}\left(\mathbf{N}^{d}\right), \ell^{2}\left(\mathbf{N}^{d}\right), \ell_{[1 / \vartheta]}^{p^{\prime}}\left(\mathbf{N}^{d}\right)\right) & \stackrel{T_{1}}{\rightarrow}( \\
T_{2} \searrow & \left(\mathcal{H}_{[\vartheta]}^{p}\left(\mathbf{R}^{d}\right), L^{2}\left(\mathbf{R}^{d}\right), \mathcal{H}_{[1 / \vartheta]}^{p^{\prime}}\left(\mathbf{R}^{d}\right)\right) \\
\downarrow & \mathfrak{V}_{d} \\
& \left(\mathcal{A}_{[\vartheta]}^{p}\left(\mathbf{C}^{d}\right), A^{2}\left(\mathbf{C}^{d}\right), \mathcal{A}_{[1 / \vartheta]}^{p^{\prime}}\left(\mathbf{C}^{d}\right)\right)
\end{aligned}
$$

are isometric bijections for every weight $\vartheta$ on $\mathbf{N}^{d}$ and $p \in(0, \infty]$. Note that the latter triples do not necessarily need to be Gelfand-triples. On the other hand, if more restrictive $p \in[1, \infty)$ and $\vartheta>c$ for some constant $c>0$, then the triples in (2.13) are Gelfand-triples.

2.2. Identification properties between $\mathcal{A}_{[\vartheta]}^{2}\left(\mathbf{C}^{d}\right)$ and $A_{(\omega)}\left(\mathbf{C}^{d}\right)$. Any formal power series (2.4) may be identified with the function

$$
F(z)=\sum_{\alpha \in \mathbf{N}^{d}} c_{\alpha} \frac{z^{\alpha}}{\sqrt{\alpha !}},
$$

provided the series on the right-hand side converges in a neighborhood of origin. Then $F(z) \in A_{d}\{0\}$. More precisely, the map

$$
\begin{aligned}
\sum_{\alpha \in \mathbf{N}^{d}} c_{\alpha} e_{\alpha} & \mapsto\left(z \mapsto \sum_{\alpha \in \mathbf{N}^{d}} c_{\alpha} \frac{z^{\alpha}}{\sqrt{\alpha !}}\right) \\
\mathcal{H}_{0}\left(\mathbf{R}^{d}\right) & \rightarrow A\left(\mathbf{C}^{d}\right)
\end{aligned}
$$

is injective and continuous. Since two different power series give rise to two different functions in $A_{d}\{0\}$, and that every function in $A_{d}\{0\}$ is equal to its power series near origin, it follows that the map in (2.14) extends to a bijective and continuous map from the set of power series which are convergent near origin to $A_{d}\{0\}$. From now on we often identify power series which converges near origin with corresponding functions in $A_{d}\{0\}$.

Next we show that (2.14) extends to a bijective and isometric map from $\mathcal{A}_{[\vartheta]}^{2}\left(\mathbf{C}^{d}\right)$ to $A_{(\omega)}^{2}\left(\mathbf{C}^{d}\right)$ and its norm, when $\vartheta$ and $\omega$ are given by

$$
\vartheta(\alpha)=\left(\frac{1}{\alpha !} \int_{\mathbf{R}_{+}^{d}} \omega_{0}(r)^{2} r^{\alpha} d r\right)^{\frac{1}{2}}
$$


and

$$
\omega(z)=e^{\frac{|z|^{2}}{2}} \omega_{0}\left(\left|z_{1}\right|^{2}, \ldots,\left|z_{d}\right|^{2}\right)
$$

for some suitable weight $\omega_{0}$ on $\mathbf{R}_{+}^{d}$. Consequently, the Bargmann transform is bijective and isometric from $\mathcal{H}_{[\vartheta]}^{2}\left(\mathbf{R}^{d}\right)$ to $A_{(\omega)}^{2}\left(\mathbf{C}^{d}\right)$ for such choices of $\vartheta$ and $\omega$. See also [18, Theorem (4.1)] for related results in one dimension.

Theorem 2.5. Let $e_{\alpha}$ be as in (1.20), $\alpha \in \mathbf{N}^{d}$, and let $\omega_{0}$ be a positive measurable function on $\mathbf{R}_{+}^{d}$. Also let $\vartheta$ and $\omega$ be weights on $\mathbf{N}^{d}$ and $\mathbf{C}^{d}$, respectively, related to each others by (2.15) and (2.16), and such that

$$
\frac{r^{|\alpha|}}{(\alpha !)^{\frac{1}{2}}} \lesssim \vartheta(\alpha), \quad \alpha \in \mathbf{N}^{d}
$$

holds for every $r>0$. Then the map (2.14) extends uniquely to a bijective and isometric map from $\mathcal{A}_{[\vartheta]}^{2}\left(\mathbf{C}^{d}\right)$ to $A_{(\omega)}^{2}\left(\mathbf{C}^{d}\right)$. In particular, the following is true:

(1) $A_{(\omega)}^{2}\left(\mathbf{C}^{d}\right)$ is a Hilbert space with $\left\{\frac{e_{\alpha}}{\vartheta(\alpha)}\right\}_{\alpha \in \mathbf{N}^{d}}$ is an orthonormal basis;

(2) $\mathfrak{V}_{d}$ from $\mathcal{H}_{0}\left(\mathbf{R}^{d}\right)$ to $A\left(\mathbf{C}^{d}\right)$, extends uniquely to a bijective and isometric map from $\mathcal{H}_{[\vartheta]}^{2}\left(\mathbf{R}^{d}\right)$ to $A_{(\omega)}^{2}\left(\mathbf{C}^{d}\right)$, and

$$
\left\|\mathfrak{V}_{d} f\right\|_{A_{(\omega)}^{2}}=\left(\sum_{\alpha}\left|c_{\alpha}(f) \vartheta(\alpha)\right|^{2}\right)^{\frac{1}{2}},
$$

when $c_{\alpha}(f)$ is given by (2.10).

Proof. Let $F \in \mathcal{A}_{[\vartheta]}^{2}\left(\mathbf{C}^{d}\right)$. By (2.17) it follows that the power series expansion of $F$ is absolutely convergent for every $z \in \mathbf{C}^{d}$. Hence, $F \in A\left(\mathbf{C}^{d}\right)$, which implies that $\mathcal{A}_{[\vartheta]}^{2}\left(\mathbf{C}^{d}\right) \subseteq A\left(\mathbf{C}^{d}\right)$. When proving that $A_{(\omega)}^{2}\left(\mathbf{C}^{d}\right)=\mathcal{A}_{[\vartheta]}^{2}\left(\mathbf{C}^{d}\right)$, it suffices to prove (1) and (2), in view of the definition of $\mathcal{A}_{[\vartheta]}^{2}$ norm, since any entire function is equal to its power series expansion.

We have

$$
\left(e_{\alpha}, e_{\beta}\right)_{A_{(\omega)}^{2}}=\pi^{-d} \int_{\mathbf{C}^{d}} \frac{z^{\alpha} \bar{z}^{\beta}}{(\alpha ! \beta !)^{\frac{1}{2}}} \omega_{0}\left(\left|z_{1}\right|^{2}, \ldots,\left|z_{d}\right|^{2}\right)^{2} d \lambda(z) .
$$

Let

$$
\begin{aligned}
z=\left(r_{1} e^{i \varphi_{1}}, \ldots, r_{d} e^{i \varphi_{d}}\right), \quad \text { where } \quad r & =\left(r_{1}, \ldots, r_{d}\right) \in[0, \infty)^{d}, \\
\text { and } \varphi & =\left(\varphi_{1}, \ldots, \varphi_{d}\right) \in[0,2 \pi)^{d} .
\end{aligned}
$$


Then the last integral becomes

$$
\pi^{-d} \int_{\mathbf{R}_{+}^{d}} \int_{[0,2 \pi)^{d}} \frac{r^{\alpha+\beta} e^{i\langle\alpha-\beta, \varphi\rangle}}{(\alpha ! \beta !)^{\frac{1}{2}}} \omega_{0}\left(r_{1}^{2}, \ldots, r_{d}^{2}\right)^{2} r_{1} \cdots r_{d} d \varphi d r .
$$

By the assumptions it follows that the last integral belongs to $L^{1}\left([0, \infty)^{d} \times\right.$ $\left.[0,2 \pi)^{d}\right)$. Hence, by Fubbini's theorem we get

$$
\begin{gathered}
\pi^{-d} \int_{\mathbf{R}_{+}^{d}} \int_{[0,2 \pi)^{d}} \frac{r^{\alpha+\beta} e^{i\langle\alpha-\beta, \varphi\rangle}}{(\alpha ! \beta !)^{\frac{1}{2}}} \omega_{0}\left(r_{1}^{2}, \ldots, r_{d}^{2}\right)^{2} r_{1} \cdots r_{d} d \varphi d r \\
=\pi^{-d} \int_{\mathbf{R}_{+}^{d}}\left(\int_{[0,2 \pi)^{d}} e^{i\langle\alpha-\beta, \varphi\rangle} d \varphi\right) \frac{r^{\alpha+\beta}}{(\alpha ! \beta !)^{\frac{1}{2}}} \omega_{0}\left(r_{1}^{2}, \ldots, r_{d}^{2}\right)^{2} r_{1} \cdots r_{d} d r \\
=2^{d} \delta_{\alpha, \beta} \int_{\mathbf{R}_{+}^{d}} \frac{r^{2 \alpha}}{\alpha !} \omega_{0}\left(r_{1}^{2}, \ldots, r_{d}^{2}\right) r_{1} \cdots r_{d} d r \\
=\delta_{\alpha, \beta} \frac{1}{\alpha !} \int_{\mathbf{R}_{+}^{d}} r^{\alpha} \omega_{0}(r)^{2} d r=\delta_{\alpha, \beta} \vartheta(\alpha)^{2} .
\end{gathered}
$$

Hence, $\left\{\frac{e_{\alpha}}{\vartheta(\alpha)}\right\}_{\alpha \in \mathbf{N}^{d}}$ is an orthonormal sequence in $A_{(\omega)}^{2}\left(\mathbf{C}^{d}\right)$, and

$$
\sum_{\alpha}\left|c_{\alpha} \vartheta(\alpha)\right|^{2} \leq\|F\|_{A_{(\omega)}^{2}},
$$

when $F$ is given by (2.4).

For general $F \in A_{(\omega)}^{2}\left(\mathbf{C}^{d}\right)$ with Taylor series (2.4), we have

$$
\begin{aligned}
& \|F\|_{A_{(\omega)}^{2}}^{2}=\pi^{-d} \int_{\mathbf{C}^{d}}\left|F(z) \omega_{0}\left(\left|z_{1}\right|^{2}, \ldots,\left|z_{d}\right|^{2}\right)\right|^{2} d \lambda(z) \\
& \quad=\pi^{-d} \int_{\mathbf{R}_{+}^{d}}\left(\int_{[0,2 \pi)^{d}} \Phi(r, \varphi) d \varphi\right) \omega_{0}\left(r_{1}^{2}, \ldots, r_{d}^{2}\right)^{2} r_{1} \cdots r_{d} d r
\end{aligned}
$$

where

$$
\Phi(r, \varphi)=\sum_{\alpha, \beta \in \mathbf{N}^{d}} \frac{c_{\alpha} \overline{c_{\beta}} r^{\alpha+\beta} e^{i\langle\alpha-\beta, \varphi\rangle}}{(\alpha ! \beta !)^{\frac{1}{2}}} .
$$

By (2.17) and (2.19), it follows that the radius of convergence of $r_{j} \mapsto$ $\Phi(r, \varphi), j=1, \ldots, d$, is equal to $\infty$ (with uniform bounds with respect to $\left.\varphi \in[0,2 \pi)^{d}\right)$. This gives

$$
\begin{aligned}
\int_{[0,2 \pi)^{d}} & \left(\sum_{\alpha, \beta \in \mathbf{N}^{d}} \frac{c_{\alpha} \overline{c_{\beta}} r^{\alpha+\beta} e^{i\langle\alpha-\beta, \varphi\rangle}}{(\alpha ! \beta !)^{\frac{1}{2}}}\right) d \varphi \\
& =\sum_{\alpha, \beta \in \mathbf{N}^{d}} \int_{[0,2 \pi)^{d}} \frac{c_{\alpha} \overline{c_{\beta}} r^{\alpha+\beta} e^{i\langle\alpha-\beta, \varphi\rangle}}{(\alpha ! \beta !)^{\frac{1}{2}}} d \varphi=(2 \pi)^{d} \sum_{\alpha \in \mathbf{N}^{d}} \frac{\left|c_{\alpha}\right|^{2} r^{2 \alpha}}{\alpha !},
\end{aligned}
$$


and by (2.20) we get

$$
\begin{aligned}
\|F\|_{A_{(\omega)}^{2}}^{2}=2^{d} & \int_{\mathbf{R}_{+}^{d}}\left(\sum_{\alpha \in \mathbf{N}^{d}} \frac{\left|c_{\alpha}\right|^{2} r^{2 \alpha}}{\alpha !}\right) \omega_{0}\left(r_{1}^{2}, \ldots, r_{d}^{2}\right)^{2} r_{1} \cdots r_{d} d r \\
& =\int_{\mathbf{R}_{+}^{d}}\left(\sum_{\alpha \in \mathbf{N}^{d}} \frac{\left|c_{\alpha}\right|^{2} r^{\alpha}}{\alpha !}\right) \omega_{0}\left(r_{1}, \ldots, r_{d}\right)^{2} d r \\
& =\sum_{\alpha \in \mathbf{N}^{d}} \frac{\left|c_{\alpha}\right|^{2}}{\alpha !} \int_{\mathbf{R}_{+}^{d}} r^{\alpha} \omega_{0}\left(r_{1}, \ldots, r_{d}\right)^{2} d r=\sum_{\alpha \in \mathbf{N}^{d}}\left|c_{\alpha} \vartheta(\alpha)\right|^{2} .
\end{aligned}
$$

Hence $F \in \mathcal{A}_{[\theta]}^{2}\left(\mathbf{C}^{d}\right)$ and $\|F\|_{A_{(\omega)}^{2}}=\|F\|_{\mathcal{A}_{[\theta]}^{2}}$. This gives the result.

Remark 2.6. We note that for any $F \in A\left(\mathbf{C}^{d}\right)$, then $F \in A_{(\omega)}^{2}\left(\mathbf{C}^{d}\right)$ for some choice of $\omega$. This follows by letting

$$
\omega(r)=\left(\sup _{|w|=|r|}|F(w)|+1\right)^{-1} e^{-|r|} .
$$

A common situation is that $\omega$ in Theorem 2.5 is radial symmetric. In this case, (2.16) takes the forms

$$
\omega(z)=e^{\frac{|z|^{2}}{2}} \omega_{0}\left(|z|^{2}\right),
$$

for some weight $\omega_{0}$ on $\overline{\mathbf{R}_{+}}$, and in the following proposition we show that $\vartheta(\alpha)$ becomes

$$
\vartheta(\alpha)=\left(\frac{1}{(|\alpha|+d-1) !} \int_{0}^{\infty} \omega_{0}(r)^{2} r^{|\alpha|+d-1} d r\right)^{\frac{1}{2}} .
$$

In particular, $\vartheta(\alpha)$ is also radial symmetric with respect to $\alpha$.

Proposition 2.7. Let $\omega_{0}$ be a weight on $\overline{\mathbf{R}_{+}}$, and let $\vartheta$ and $\omega$ be weights on $\mathbf{N}^{d}$ and $\mathbf{C}^{d}$, respectively, related to each others by $(2.15)^{\prime}$ and $(2.16)^{\prime}$, and such that (2.17) holds for every $R>0$. Then the map (2.14) extends uniquely to a bijective and isometric map from $\mathcal{A}_{[\vartheta]}^{2}\left(\mathbf{C}^{d}\right)$ to $A_{(\omega)}^{2}\left(\mathbf{C}^{d}\right)$.

Proof. Let

$\Delta_{j}=\left\{\left(t_{1}, \ldots, t_{d-j}\right) \in[0,1]^{d-j} ; t_{1}+\cdots+t_{d-j} \leq 1\right\}, \quad j=1, \ldots, d-1$.

We take $r \in \mathbf{R}_{+}$and $\left(t_{1}, \ldots, t_{d-1}\right) \in \Delta_{1}$ as new variables of integration in (2.15), where

$$
r_{j}=r t_{j}, \text { when } j \neq d \quad \text { and } \quad r_{d}=r\left(1-\sum_{j=1}^{d-1} t_{j}\right) .
$$


By straight-forward computations it follows that the Jacobian is equal to $r^{d-1}$. Hence, (2.15) gives that $\vartheta(\alpha)^{2}=I \cdot J$, where

$$
I=\frac{1}{\alpha !} \int_{\mathbf{R}_{+}} \omega(r)^{2} r^{|\alpha|+d-1} d r
$$

and

$$
J=\int_{\Delta_{1}}\left(\prod_{j=1}^{d-1} t_{j}^{\alpha_{j}}\right)\left(1-\sum_{j=1}^{d-1} t_{j}\right)^{\alpha_{d}} d t
$$

We set

$$
J_{k}=c_{k} \int \cdots \int_{\Delta_{k}}\left(\prod_{j=1}^{d-k} t_{j}^{\alpha_{j}}\right)\left(1-\sum_{j=1}^{d-k} t_{j}\right)^{\beta_{k}} d t_{1} \cdots d t_{d-k}
$$

where

$$
\beta_{k}=\left(\sum_{j=d-k+1}^{d} \alpha_{j}\right)+k-1 \text { and } c_{k}=\left(\beta_{k} !\right)^{-1}\left(\prod_{j=d-k+1}^{d} \alpha_{j} !\right) .
$$

We have $J=J_{1}$, and claim that $J=J_{k}$ for $k=1, \ldots, d-1$, or equivalently, $J_{k}=J_{k+1}, k=1, \ldots, d-2$.

In fact, let

$$
y_{k}=\left(t_{1}, \ldots, t_{d-k-1}\right) \in \Delta_{k+1}, \quad \gamma_{k}=\left(\alpha_{1}, \ldots, \alpha_{d-k-1}\right)
$$

and

$$
\varphi\left(y_{k}\right)=1-\sum_{j=1}^{d-k-1} t_{j}
$$

Then

$$
J_{k}=c_{k} \int_{\Delta_{k+1}} y_{k}^{\gamma_{k}} h\left(y_{k}\right) d y_{k}
$$

where

$$
h\left(y_{k}\right)=\int_{0}^{\varphi\left(y_{k}\right)} u^{\alpha_{d-k}}\left(\varphi\left(y_{k}\right)-u\right)^{\beta_{k}} d u
$$

By integrations by parts we get

$$
\begin{gathered}
h\left(y_{k}\right)=\int_{0}^{\varphi\left(y_{k}\right)} u^{\alpha_{d-k}}\left(\varphi\left(y_{k}\right)-u\right)^{\beta_{k}} d u \\
=\frac{\alpha_{d-k} !}{\left(\beta_{k}+1\right) \cdots\left(\beta_{k}+\alpha_{d-k}\right)} \int_{0}^{\varphi\left(y_{k}\right)}\left(\varphi\left(y_{k}\right)-u\right)^{\beta_{k}+\alpha_{d-k}} d u \\
\left.=\alpha_{d-k} ! \beta_{k} !\left(\beta_{k}+\alpha_{d-k}+1\right) !\right)^{-1} \varphi\left(y_{k}\right)^{\beta_{k}+\alpha_{d-k}+1}=\alpha_{d-k} ! \beta_{k} !\left(\beta_{k+1} !\right)^{-1} \varphi\left(y_{k}\right)^{\beta_{k+1}} \\
{ }_{26}
\end{gathered}
$$


and inserting this into (2.23) gives

$$
J_{k}=c_{k} \alpha_{d-k} ! \beta_{k} !\left(\beta_{k+1} !\right)^{-1} \int_{\Delta_{k+1}} y^{\gamma_{k}} \varphi\left(y_{k}\right)^{\beta_{k+1}} d y_{k}=J_{k+1} .
$$

Hence, by integration by parts we obtain

$$
\begin{gathered}
J=J_{d-1}=c_{d-1} \int_{0}^{1} t_{1}^{\alpha_{1}}\left(1-t_{1}\right)^{\beta_{d-1}} d t_{1} \\
=c_{d-1} \alpha_{1} ! \beta_{d-1} !\left(\left(\beta_{d-1}+\alpha_{1}\right) !\right)^{-1} \int_{0}^{1}\left(1-t_{1}\right)^{\beta_{d-1}+\alpha_{1}} d t_{1} \\
\quad=c_{d-1} \alpha_{1} ! \beta_{d-1} !\left(\left(\beta_{d-1}+\alpha_{1}+1\right) !\right)^{-1}=\frac{\alpha !}{\beta_{d} !} .
\end{gathered}
$$

The result now follows by from the latter equalities, (2.21) and the fact that $\vartheta(\alpha)^{2}=I \cdot J$.

A simple but interesting case appears when $\omega=\omega_{h}$ in Proposition 2.7 is given by

$$
\omega_{h}(z)=e^{\frac{1}{2}(1-2 h)|z|^{2}} .
$$

Corollary 2.8. Let $F \in A\left(\mathbf{C}^{d}\right)$ be given by (2.4), $\omega_{h}$ be given by (2.24), and let $\vartheta_{r}(\alpha)=e^{r|\alpha|}$ when $r \in \mathbf{R}$ and $\alpha \in \mathbf{N}^{d}$.

$$
\|F\|_{A_{\left(\omega_{h}\right)}^{2}}=e^{r d}\left(\sum_{\alpha}\left|c_{\alpha} e^{r|\alpha|}\right|^{2}\right)^{\frac{1}{2}}, \quad r=-\frac{\log (2 h)}{2} .
$$

Furthermore, the map $\mathfrak{V}_{d}$ from $\mathcal{H}_{0}\left(\mathbf{R}^{d}\right)$ to $A\left(\mathbf{C}^{d}\right)$ extends uniquely to a homeomorphism from $\mathcal{H}_{\left[\vartheta_{r}\right]}^{2}\left(\mathbf{R}^{d}\right)$ to $A_{\left(\omega_{h}\right)}^{2}\left(\mathbf{C}^{d}\right)$, and

$$
\left\|\mathfrak{V}_{d} f\right\|_{A_{\left(\omega_{h}\right)}^{2}}=e^{r d}\|f\|_{\mathcal{H}_{\left[\vartheta_{r}\right]}^{2}}, \quad r=-\frac{\log (2 h)}{2} .
$$

Proof. Let $F \in A\left(\mathbf{C}^{d}\right), \omega_{0}(r)=e^{-h\left(t_{1}+\cdots+t_{d}\right)}$ and let $\vartheta$ be as in Theorem 2.5. Then

$$
\begin{aligned}
\vartheta(\alpha)^{2} & =\frac{1}{\alpha !} \int_{\mathbf{R}_{+}^{d}} \omega_{0}(t)^{2} t^{\alpha} d t=\frac{1}{\alpha !} \int_{\mathbf{R}_{+}^{d}} e^{-2 h\left(t_{1}+\cdots+t_{d}\right)} t^{\alpha} d t \\
& =(2 h)^{-|\alpha|-d} \frac{1}{\alpha !} \int_{\mathbf{R}_{+}^{d}} e^{-\left(t_{1}+\cdots+t_{d}\right)} r^{\alpha} d t=(2 h)^{-|\alpha|-d}=e^{2 r d} \vartheta_{r}(\alpha)^{2} .
\end{aligned}
$$

The result now follows from Theorem 2.5 or Proposition 2.7 ,

3. Characterizations of $\mathcal{H}_{b_{\sigma}}\left(\mathbf{R}^{d}\right)$ And $\mathcal{H}_{0, b_{\sigma}}\left(\mathbf{R}^{d}\right)$, And their

$$
\text { DUALS }
$$

In this section we consider $\mathcal{H}_{b_{\sigma}}\left(\mathbf{R}^{d}\right), \mathcal{H}_{0, b_{\sigma}}\left(\mathbf{R}^{d}\right), \mathcal{H}_{b_{\sigma}}^{\prime}\left(\mathbf{R}^{d}\right)$ and $\mathcal{H}_{0, b_{\sigma}}^{\prime}\left(\mathbf{R}^{d}\right)$, and their images under the Bargmann transform. We show that the Barmann transform is continuous and bijective from $\mathcal{H}_{b_{1}}^{\prime}\left(\mathbf{R}^{d}\right)$ to $A\left(\mathbf{C}^{d}\right)$, 
and from $\mathcal{H}_{0, b_{1}}^{\prime}\left(\mathbf{R}^{d}\right)$ to $A_{d}\{0\}$. Furthermore, we prove that the images of $\mathcal{H}_{b_{\sigma}}^{\prime}\left(\mathbf{R}^{d}\right)$ and $\mathcal{H}_{0, b_{\sigma}}^{\prime}\left(\mathbf{R}^{d}\right)$ for $\sigma>1$, and $\mathcal{H}_{b_{\sigma}}\left(\mathbf{R}^{d}\right)$ and $\mathcal{H}_{0, b_{\sigma}}\left(\mathbf{R}^{d}\right)$ for any $\sigma>0$ are sets of entire functions on $\mathbf{C}^{d}$, obeying suitable boundedness conditions of exponential types. (Cf. Theorems 3.1 and 3.2.)

We use these properties to extend the definition of modulation spaces to permit any weight function, and show that these spaces are quasiBanach spaces, and thereby complete.

In the last part of the section we analyze the test function space $\mathcal{S}_{C}\left(\mathbf{R}^{d}\right)$, introduced by Gröchenig in [15], and show that this space agrees with $\mathcal{H}_{0, b_{1}}\left(\mathbf{R}^{d}\right)$.

The following results identify the spaces in (2.9) in the case $s=b_{\sigma}$ with convenient topological spaces of analytic functions. The first one deals with the images of test function spaces, and the second one with corresponding distribution spaces. Here recall that $b_{\infty}=\frac{1}{2}$, and set

$$
\kappa_{1}(\sigma)=\left\{\begin{array}{ll}
\frac{2 \sigma}{\sigma+1}, & 0 \leq \sigma<\infty \\
2, & \sigma=\infty
\end{array} \quad \text { and } \quad \kappa_{2}(\sigma)= \begin{cases}\frac{2 \sigma}{\sigma-1}, & 1<\sigma<\infty \\
2, & \sigma=\infty .\end{cases}\right.
$$

Theorem 3.1. Let $\kappa_{1}$ be given by (3.1). Then the following is true:

(1) if $\sigma \in(0, \infty]$, then the map (2.14) extends uniquely to a homeomorphism from $\mathcal{A}_{0, b_{\sigma}}\left(\mathbf{C}^{d}\right)$ to

$$
\left\{F \in A\left(\mathbf{C}^{d}\right) ;|F(z)| \lesssim e^{r|z|^{\kappa_{1}(\sigma)}} \text { for every } r>0\right\}
$$

(2) if $\sigma \in(0, \infty)$, then the map (2.14) extends uniquely to a homeomorphism from $\mathcal{A}_{b_{\sigma}}\left(\mathbf{C}^{d}\right)$ to

$$
\left\{F \in A\left(\mathbf{C}^{d}\right) ;|F(z)| \lesssim e^{r|z|^{\kappa_{1}(\sigma)}} \text { for some } r>0\right\} .
$$

Theorem 3.2. Let $\kappa_{2}$ be given by (3.1). Then the following is true:

(1) the map (2.14) extends uniquely to a homeomorphism from $\mathcal{A}_{b_{1}}^{\prime}\left(\mathbf{C}^{d}\right)$ to $A\left(\mathbf{C}^{d}\right)$, and from $\mathcal{A}_{0, b_{1}}^{\prime}\left(\mathbf{C}^{d}\right)$ to $A_{d}\{0\}$;

(2) if $\sigma \in(1, \infty)$, then the map (2.14) extends uniquely to a homeomorphism from $\mathcal{A}_{b_{\sigma}}^{\prime}\left(\mathbf{C}^{d}\right)$ to

$$
\left\{F \in A\left(\mathbf{C}^{d}\right) ;|F(z)| \lesssim e^{r|z|^{\kappa_{2}(\sigma)}} \text { for every } r>0\right\}
$$

(3) if $\sigma \in(1, \infty]$, then the map (2.14) extends uniquely to a homeomorphism from $\mathcal{A}_{0, b_{\sigma}}^{\prime}\left(\mathbf{C}^{d}\right)$ to

$$
\left\{F \in A\left(\mathbf{C}^{d}\right) ;|F(z)| \lesssim e^{r|z|^{\kappa_{2}(\sigma)}} \text { for some } r>0\right\} .
$$

We recall that Remark 1.12 explains the topologies for the sets in (3.2)- (3.5).

We need some preparations for the proof and start to make some remarks on the topologies of $A\left(\mathbf{C}^{d}\right)$ and $A_{d}\{0\}$. Let $r_{0}=\left(r_{0,1}, \ldots, r_{0, d}\right) \in$ $[0, \infty)^{d}$ and $F$ be defined in the open neighbourhood $\Omega \subseteq D_{r_{0}}(0) \subseteq \mathbf{C}^{d}$ 
of origin. Then $F$ is extendable to an analytic function in $A\left(D_{r}(0)\right)$, if and only if (2.4) holds when $z \in \Omega$ for some $\left\{c_{\alpha}\right\}_{\alpha \in \mathbf{N}^{d}}$ which satisfies

$$
\sup _{\alpha}\left|\frac{c_{\alpha} r^{\alpha}}{(\alpha !)^{\frac{1}{2}}}\right|<\infty
$$

for every $r \in[0, \infty)^{d}$ such that $r<r_{0}$. Here $\left(r_{1}, \ldots, r_{d}\right)<\left(r_{0,1}, \ldots, r_{0, d}\right)$ means that $r_{j}<r_{0, j}$ for every $j=1, \ldots, d$.

The map which takes the power series in (2.4) into the left-hand side of (3.6) defines a semi-norm on $A\left(D_{r_{0}}(0)\right)$, for every $r<r_{0}$. It follows that $A\left(D_{r_{0}}(0)\right)$ is a Fréchet space under the topology defined by these semi-norms. Now we let the topologies in $A\left(\mathbf{C}^{d}\right)$ and $A_{d}\{0\}$ be defined as the projective limit and inductive limit topology, respectively, of $A\left(D_{r}(0)\right), r>0$.

In the following propositions we let

$$
\omega_{r, \sigma}(x, \xi)=e^{\frac{1}{4}\left(|x|^{2}+|\xi|^{2}\right)} e^{-r\left(|x|^{\kappa_{1}(\sigma)}+|\xi|^{\kappa_{1}(\sigma)}\right)},
$$

Proposition 3.3. Let $p \in(0, \infty], \sigma \in(0, \infty), \kappa_{1}$ be as in (3.1), $\mathscr{B}$ be a mixed quasi-norm space, $\phi$ be as in (1.14), $f \in \mathcal{S}_{\frac{1}{2}}^{\prime}\left(\mathbf{R}^{d}\right)$, and let $F=\mathfrak{V}_{d} f$. Also let $\omega_{r, \sigma}$ be as in (3.7). Then the following conditions are equivalent:

(1) $f \in \mathcal{H}_{b_{\sigma}}\left(\mathbf{R}^{d}\right)$;

(2) $\left\|F \cdot e^{-r|\cdot|^{\kappa_{1}(\sigma)}}\right\|_{L^{p}}<\infty$ for some $r>0$;

(3) $f \in M\left(\omega_{r, \sigma}, \mathscr{B}\right)$ for some $r>0$.

Proposition 3.4. Let $p \in(0, \infty], \sigma \in(0, \infty], \kappa_{1}$ be as in (3.1), $\mathscr{B}$ be a mixed quasi-norm space, $\phi$ be as in (1.14), $f \in \mathcal{S}_{\frac{1}{2}}^{\prime}\left(\mathbf{R}^{d}\right)$, and let $F=\mathfrak{V}_{d} f$. Also let $\omega_{r, \sigma}$ be as in (3.7). Then the following conditions are equivalent:

(1) $f \in \mathcal{H}_{0, b_{\sigma}}\left(\mathbf{R}^{d}\right)$;

(2) $\left\|F \cdot e^{-r|\cdot|^{\kappa_{1}(\sigma)}}\right\|_{L^{p}}<\infty$ for every $r>0$;

(3) $f \in M\left(\omega_{r, \sigma}, \mathscr{B}\right)$ for every $r>0$.

Proof of Propositions 3.3 and 3.4. If $\mathscr{B}=L^{p}$, then (2) is equivalent to (3), in view of the definitions. Since $\left\{\omega_{R, \sigma}\right\}_{R>0}$ is an admissible family of weights, Theorem 3.4 in [26] shows that (2) and (3) are equivalent for arbitrary $\mathscr{B}$. We need to prove that (2) is equivalent to (1), and then we first assume that (1) Proposition 3.3 holds.

Let $C$ and $R$ be such that

$$
\left|c_{\alpha}(f)\right| \leq C R^{|\alpha|}(\alpha !)^{-\frac{1}{2 \sigma}},
$$


and let $r=|z|$ and $\rho=\frac{1}{\kappa_{1}(\sigma)}$. Then

$$
\begin{aligned}
|F(z)|=\left|\sum_{\alpha} c_{\alpha}(f) \frac{z^{\alpha}}{(\alpha !)^{\frac{1}{2}}}\right| \leq C \sum_{\alpha} \frac{\left(R_{1} r\right)^{|\alpha|}}{(\alpha !)^{\rho}} & \\
& \lesssim \sup _{k \geq 0} \frac{\left(R_{2} r\right)^{k}}{(k !)^{\rho}} \lesssim \sup _{u \geq 1} \frac{\left(R_{3} r\right)^{u}}{u^{\rho u}},
\end{aligned}
$$

for some $R_{j}, j \geq 1$.

We need to maximize

$$
g(u)=\ln \left(\frac{r_{1}^{u}}{u^{\rho u}}\right)=u \ln r_{1}-\rho u \ln u, \quad u \geq 1,
$$

where $r_{1}=R_{3} r$. By differentiation it follows that the maximum is attained for

giving that

$$
u=u_{0}=\frac{r_{1}^{\frac{1}{\rho}}}{e}
$$

$$
|F(z)| \lesssim e^{g\left(u_{0}\right)}=e^{\rho u_{0}}=e^{c|z|^{\kappa_{1}(\sigma)}},
$$

for some constant $c>0$. This gives (2) in Proposition 3.3 .

From these arguments it also follows that that there is a constant $C>0$ such that $c$ can be chosen in such way that

$$
C^{-1} \leq R c \leq C
$$

which shows that Proposition 3.4 (1) implies Proposition 3.4 (2), also in the case $\sigma=\infty$.

Assume instead that (2) in Proposition 3.3 holds, let $c_{\alpha}$ be chosen such that (2.4) holds, $k=|\alpha|+d-1$ and let $\vartheta(\alpha)$ be given by $(2.15)^{\prime}$. Then

$$
\vartheta(\alpha)^{2}=\frac{1}{k !} \int_{0}^{\infty} e^{-2 c r^{\frac{1}{2 \rho}}} r^{k} d r .
$$

By taking $u=2 c r^{\frac{1}{2 \rho}}$ as new variable of integration we obtain

$$
\vartheta(\alpha)^{2}=\frac{2 \rho}{\left.(2 c)^{2 \rho(k+1}\right) k !} \int_{0}^{\infty} e^{-u} u^{2 \rho(k+1)-1} d u \asymp \frac{\Gamma(2 \rho(k+1))}{(2 c)^{2 \rho k} k !} .
$$

By Stirling's formula we get

$$
\begin{aligned}
\vartheta(\alpha)^{2} \asymp \theta_{1}^{k} \frac{\left(\frac{2 \rho(k+1)-1}{e}\right)^{2 \rho(k+1)-1}}{\left(\frac{k}{e}\right)^{k}} & \gtrsim \theta_{2}^{k} k^{(2 \rho-1) k} \\
& =\theta_{2}^{k} k^{\frac{k}{\sigma}} \gtrsim \theta_{3}^{k}(k !)^{\frac{1}{\sigma}} \gtrsim \theta_{4}^{|\alpha|}(\alpha !)^{\frac{1}{\sigma}},
\end{aligned}
$$

for some constants $\theta_{j}, j=1, \ldots, 4$. 
If $\theta=\theta_{4}^{\frac{1}{2}}$, then Theorem 2.5 now gives

$$
\sum_{\alpha}\left|c_{\alpha} \theta^{|\alpha|}(\alpha !)^{\frac{1}{2 \sigma}}\right|^{2} \lesssim \sum_{\alpha}\left|c_{\alpha} \vartheta(\alpha)\right|^{2} \asymp\left\|F \cdot e^{-c|\cdot|^{\frac{1}{\rho}}}\right\|_{L^{2}}<\infty,
$$

provided $c>$ is chosen large enough. This gives (1) in Proposition 3.3 .

Evidently, the constants $\theta_{j}$ here above can be chosen in such way that

$$
C^{-1} \leq \theta_{j} c \leq C,
$$

for some constant $C>0$ which is independent of $c$, which shows that (2) in Proposition 3.4 implies (1) in Proposition 3.4 when $\sigma<\infty$. By similar arguments, or using Corollary 2.8, it follows that the latter implication also holds in the case $\sigma=\infty$.

For the duals $\mathcal{H}_{b_{\sigma}}^{\prime}\left(\mathbf{R}^{d}\right)$ and $\mathcal{H}_{0, b_{\sigma}}^{\prime}\left(\mathbf{R}^{d}\right)$ we have now the following.

Proposition 3.5. Let $p \in[1, \infty], \sigma \in(1, \infty), \kappa_{2}$ be as in (3.1), $\phi$ be as in (1.14), $f \in \mathcal{H}_{0}^{\prime}\left(\mathbf{R}^{d}\right)$, and let $F=\mathfrak{V}_{d} f$. Then the following conditions are equivalent:

(1) $f \in \mathcal{H}_{b_{\sigma}}^{\prime}\left(\mathbf{R}^{d}\right)$;

(2) $\left\|F \cdot e^{-r|\cdot|^{\kappa_{2}(\sigma)}}\right\|_{L^{p}}<\infty$ for every $r>0$.

Proposition 3.6. Let $p \in[1, \infty], \sigma \in(1, \infty], \kappa_{2}$ be as in (3.1), $\phi$ be as in (1.14),$f \in \mathcal{H}_{0}^{\prime}\left(\mathbf{R}^{d}\right)$, and let $F=\mathfrak{V}_{d} f$. Then the following conditions are equivalent:

(1) $f \in \mathcal{H}_{0, b_{\sigma}}^{\prime}\left(\mathbf{R}^{d}\right)$;

(2) $\left\|F \cdot e^{-r|\cdot|^{\kappa_{2}(\sigma)}}\right\|_{L^{p}}<\infty$ for some $r>0$.

For the proofs we need the following lemma.

Lemma 3.7. Let $R>0,0<\rho \leq \frac{1}{2}, F \in A\left(\mathbf{C}^{d}\right)$ and let $p \in[1, \infty]$. Then there are positive constants $c_{1}, c_{2}$ and $C$ which only depends on $\rho$ and $p$ such that

$$
C^{-1}\left\|F e^{-c_{1} R|\cdot|^{\frac{1}{\rho}}}\right\|_{L^{p}} \leq\left\|F e^{-R|\cdot|^{\frac{1}{\rho}}}\right\|_{L^{\infty}} \leq C\left\|F e^{-\left.c_{2} R|\cdot|\right|^{\frac{1}{\rho}}}\right\|_{L^{p}} .
$$

Proof. The first inequality in (3.10) is a straight-forward consequence of Hölder's inequality.

By Hölder's inequality again, it suffices to show that the second inequality holds for $p=1$. Let $z \in \mathbf{C}^{d}$ be fixed and let

$$
\Omega_{z}=\left\{w \in \mathbf{C}^{d} ; 2^{-1} \leq\left|w_{j}-z_{j}\right| \leq 1, j=1, \ldots, d\right\} .
$$

By the mean-value property of analytic functions we obtain

$$
\begin{aligned}
\left|F(z) e^{-R|z|^{\frac{1}{\rho}}}\right|= & \left(\frac{3 \pi}{4}\right)^{d}\left|\int_{\Omega_{z}} F(w) e^{-R|z|^{\frac{1}{\rho}}} d \lambda(w)\right| \\
& \lesssim \int_{\Omega_{z}}\left|F(w) e^{-R c|w|^{\frac{1}{\rho}}}\right| d \lambda(w) \leq\left\|F \cdot e^{-R c|\cdot| \frac{1}{\rho}}\right\|_{L^{1}\left(\mathbf{C}^{d}\right)} .
\end{aligned}
$$


By taking supremum over all $z \in \mathbf{C}^{d}$, the second inequality in (3.10) follows.

Propositions 3.5 and 3.6 follow by letting $\rho=\frac{1}{\kappa_{2}(\sigma)}$ instead of $\rho=$ $\frac{1}{\kappa_{1}(\sigma)}$ in the proof of Propositions 3.3 and 3.4, and using Lemma 3.7. The details are left for the reader.

Proof of Theorems 3.1 and 3.2. First we prove the first part of Theorem 3.2 (1), which follows if we prove that $\mathfrak{V}_{d}$ is homeomorphic from $\mathcal{H}_{b_{1}}^{\prime}\left(\mathbf{R}^{d}\right)$ to $A\left(\mathbf{C}^{d}\right)$. We have that (3.6) is true for every $r>0$, if and only if

$$
\sum_{\alpha} \frac{\left|c_{\alpha}\right|^{2} R^{|\alpha|}}{\alpha !}<\infty,
$$

for every $R>0$, and that the topology on $A\left(\mathbf{C}^{d}\right)$, induced by the latter sum, remains the same. Hence, if we let $\mathfrak{V}_{d} f$ be the power series expansion in (2.4) when $f \in \mathcal{H}_{b_{1}}^{\prime}\left(\mathbf{R}^{d}\right)$ is given by (2.10), it follows that $\mathfrak{V}_{d}$ is continuous and linear from $\mathcal{H}_{b_{1}}^{\prime}\left(\mathbf{R}^{d}\right)$ to $A\left(\mathbf{C}^{d}\right)$.

Furthermore, since the Taylor series for an analytic function is unique, it follows that $\mathfrak{V}_{d}$ is injective, and since every entire function is equal to its Taylor series, it also follows that $\mathfrak{V}_{d}$ is surjective. This gives the first part of Theorem 3.2 (1), and the second part follows by similar arguments and is left for the reader.

By (1)-(3) in Propositions 3.3 it follows that $\mathcal{A}_{\sigma_{\sigma}}\left(\mathbf{C}^{d}\right)$ is contained in (3.3). On the other hand, if $F$ belongs to the set in (3.3), which is a subset of $A\left(\mathbf{C}^{d}\right)$, then $F=\mathfrak{V}_{d} f$ for some $f \in \mathcal{H}_{b_{1}}^{\prime}\left(\mathbf{R}^{d}\right)$. By (3.9) it now follows that $f \in \mathcal{H}_{b_{\sigma}}\left(\mathbf{R}^{d}\right)$, and hence $F=\mathfrak{V}_{d} f \in \mathcal{A}_{b_{\sigma}}\left(\mathbf{C}^{d}\right)$. This gives Theorem 3.1 (2).

By using Propositions 3.4, 3.5 and 3.6 instead of Proposition 3.3, the remaining parts follow in similar ways. The details are left for the reader.

3.1. A broad family of modulation spaces. Next we show that certain parts of the modulation space theory in [8, 10, 15, 26] can be extended, by letting the modulation spaces be defined in the framework of $\mathcal{H}_{b_{1}}^{\prime}\left(\mathbf{R}^{d}\right)$. More precisely, for $\phi$ as in Definition 1.8, and $\omega$ and $\mathscr{B}$ as in Definition [1.9, we let the modulation space $M(\omega, \mathscr{B})$ be the set of $f \in \mathcal{H}_{b_{1}}^{\prime}\left(\mathbf{R}^{d}\right)$ such that (1.15) holds. This extends the notion of modulation spaces in Definition 1.8, The following result shows that 26 , Theorem 3.4] holds in a broader context.

Theorem 3.8. Let $\omega$ and $\mathscr{B}$ be as in Definition 1.9. Then the following is true:

(1) $A(\omega, \mathscr{B}), B(\omega, \mathscr{B})$ and $M(\omega, \mathscr{B})$ are quasi-Banach spaces. If in addition $\nu_{1}(\mathscr{B}) \geq 1$, then $A(\omega, \mathscr{B}), B(\omega, \mathscr{B})$ and $M(\omega, \mathscr{B})$ are Banach spaces; 
(2) the Bargmann transform is isometric and bijective from $M(\omega, \mathscr{B})$ to $A(\omega, \mathscr{B})$.

Proof. The statements in (1) concerning $B(\omega, \mathscr{B})$ are obvious. For the other spaces, we first prove that $A(\omega, \mathscr{B})$ is complete and thereafter show that (2) holds. The completeness of $A(\omega, \mathscr{B})$ is then carried over to $M(\omega, \mathscr{B})$.

Let $F_{j}, j=1,2, \ldots$ be a Cauchy sequence in $A(\omega, \mathscr{B})$ and set $G_{j, k}=$ $F_{j}-F_{k}$. Then $\left\|F_{j}-F\right\|_{B(\omega, \mathscr{B})} \rightarrow 0$ for some $F \in B(\omega, \mathscr{B})$, as $j$ tends to $\infty$. We have to prove that $F \in A\left(\mathbf{C}^{d}\right)$, and it suffices to prove that $F_{j} \rightarrow F$ locally uniformly.

We have that $\log \left|G_{j, k}(z)\right|$ is pluri-subharmonic for every $j . k \geq 1$. By using this fact and Jensen's inequality we get

$$
\begin{gathered}
\log \left|G_{j, k}(z)\right|^{p} \leq(2 \pi)^{-d} \int_{[0,2 \pi)^{d}} \log \left|G_{j, k}\left(z_{1}+r_{1} e^{i \varphi_{1}}, \ldots, z_{d}+r_{d} e^{i \varphi_{d}}\right)\right|^{p} d \varphi \\
\leq \log \left((2 \pi)^{-d} \int_{[0,2 \pi)^{d}}\left|G_{j, k}\left(z_{1}+r_{1} e^{i \varphi_{1}}, \ldots, z_{d}+r_{d} e^{i \varphi_{d}}\right)\right|^{p} d \varphi\right) .
\end{gathered}
$$

for every $r_{1}, \ldots, r_{d} \geq 0$, where $p=\nu_{1}(\mathscr{B})$. Hence,

$$
\left|F_{j}(z)-F_{k}(z)\right|^{p} \leq(2 \pi)^{-d} \int_{[0,2 \pi)^{d}}\left|G_{j, k}\left(z_{1}+r_{1} e^{i \varphi_{1}}, \ldots, z_{d}+r_{d} e^{i \varphi_{d}}\right)\right|^{p} d \varphi .
$$

By multiplying the previous inequality with $r_{1} \cdots r_{d}$ and integrating over a small rectangle $[0, \varepsilon]^{d}$ with respect to $\left(r_{1}, \ldots, r_{d}\right)$, we get

$$
\left|F_{j}(z)-F_{k}(z)\right| \leq C_{\varepsilon}\left\|F_{j}-F_{k}\right\|_{L^{p}\left(\Omega_{\varepsilon}\right)},
$$

where $C_{\varepsilon}>0$ only depends on $\varepsilon>0$. Here $\Omega_{\varepsilon}$ is the polydisc

$$
\left\{w \in \mathbf{C}^{d} ;\left|w_{j}-z_{j}\right|<\varepsilon, j=1, \ldots, d\right\} .
$$

Hence, if $\varepsilon_{1}<\varepsilon_{2}$, then

$$
\left\|F_{j}-F_{k}\right\|_{L^{\infty}\left(\Omega_{\varepsilon_{1}}\right)} \lesssim\left\|F_{j}-F_{k}\right\|_{L^{p}\left(\Omega_{\varepsilon_{2}}\right)} \lesssim\left\|F_{j}-F_{k}\right\|_{B(\omega, \mathscr{B})},
$$

where the last inequality follows from Hölder's inequality. Consequently, $F_{j}, j=1,2, \ldots$, is a Cauchy sequence in $L^{\infty}\left(\Omega_{\varepsilon_{1}}\right)$, and it follows that $F_{j} \rightarrow F_{0}$ in $L^{\infty}\left(\Omega_{\varepsilon_{1}}\right)$ for some $F_{0} \in A\left(\Omega_{\varepsilon_{1}}\right)$. Since $F_{k} \rightarrow F$ in $B(\omega, \mathscr{B})$ as $k$ tends to $\infty$, it follows, by passing to subsequences of $F_{j}$ if necessary, that $F=F_{0}$ a. e., and the analyticity of $F$ follows. This proves that $A(\omega, \mathscr{B})$ is complete.

By the definitions it follows that the Bargmann transform from $\mathcal{H}_{b_{1}}^{\prime}\left(\mathbf{R}^{d}\right)$ to $A\left(\mathbf{C}^{d}\right)$ restricts to an isometric and injective map from $M(\omega, \mathscr{B})$ to $A(\omega, \mathscr{B})$. On the other hand, if $F \in A(\omega, \mathscr{B})$, then $F=$ $\mathfrak{V}_{d} f$ for some $f \in \mathcal{H}_{b_{1}}^{\prime}\left(\mathbf{R}^{d}\right)$, in view of Theorem 3.1. By the definitions it follows that $f$ has the same (finite) quasi-norm in $M(\omega, \mathscr{B})$ as $\|F\|_{A(\omega, \mathscr{B})}$. Hence $f \in M(\omega, \mathscr{B})$, and (2) follows. This gives the result. 


\subsection{Comparisons between Pilipović spaces and a test function} space introduced by Gröchenig. We finish the section by performing comparisons between the spaces $\mathcal{H}_{b_{1}}\left(\mathbf{R}^{d}\right), \mathcal{H}_{0, b_{1}}\left(\mathbf{R}^{d}\right)$ and the space $\mathcal{S}_{C}\left(\mathbf{R}^{d}\right)$, introduced by Gröchenig in [15]. We recall that $\mathcal{S}_{C}\left(\mathbf{R}^{d}\right)$ consists of all $f \in L^{2}\left(\mathbf{R}^{d}\right)$ such that $f=V_{\phi}^{*} F$, for some $F$ belonging to $L^{\infty}\left(\mathbf{R}^{d}\right) \cap \mathscr{E}^{\prime}\left(\mathbf{R}^{d}\right)$. Here $\phi(x)=\pi^{-\frac{d}{4}} e^{-\frac{|x|^{2}}{2}}$, as usual. In particular, $f \in \mathcal{S}_{C}\left(\mathbf{R}^{d}\right)$, if and only if

$$
f(x)=(2 \pi)^{-\frac{d}{2}} \iint_{\mathbf{R}^{2 d}} F(y, \eta) e^{-\frac{1}{2}|x-y|^{2}} e^{i\langle x, \eta\rangle} d y d \eta,
$$

for some $L^{\infty}\left(\mathbf{R}^{d}\right) \cap \mathscr{E}^{\prime}\left(\mathbf{R}^{d}\right)$.

Lemma 3.9. Let $F \in L^{\infty}\left(\mathbf{C}^{d}\right)$. Then the Bargmann transform of $f=$ $V_{\phi}^{*} F$ is given by $\Pi_{A} F_{0}$, where

$$
F_{0}(x, \xi)=\left(2 \pi^{3}\right)^{\frac{d}{4}} F(\sqrt{2} x,-\sqrt{2} \xi) e^{\frac{1}{2}\left(|x|^{2}+|\xi|^{2}\right)} e^{-i\langle x, \xi\rangle} .
$$

Moreover, the image of $\mathcal{S}_{C}\left(\mathbf{R}^{d}\right)$ under the Bargmann transform is given by

$$
\left\{\Pi_{A} F ; F \in L^{\infty}\left(\mathbf{C}^{d}\right) \bigcap \mathscr{E}^{\prime}\left(\mathbf{C}^{d}\right)\right\} .
$$

Here recall that the map $\Pi_{A}$ is defined by (1.19).

Proof. The result follows by straight-forward computation of the Bargmann transform of elements given by $V_{\phi}^{*} F$ when $F \in L^{\infty}\left(\mathbf{R}^{d}\right)$ or $F \in L^{\infty}\left(\mathbf{R}^{d}\right) \cap$ $\mathscr{E}^{\prime}\left(\mathbf{R}^{d}\right)$. The details are left for the reader.

The next result links

$$
\begin{aligned}
& \Omega_{0, R}=\left\{\Pi_{A} F ; F \in L^{\infty}\left(\mathbf{C}^{d}\right), \operatorname{supp} F \subseteq B_{R}(0)\right\}, \\
& \Omega_{1, R}=\left\{\Pi_{A} F ; F \in L^{\infty}\left(\mathbf{C}^{d}\right), \operatorname{supp} F \subseteq D_{d, R}\right\}
\end{aligned}
$$

and

$$
\Omega_{2, R}=\left\{F=\sum_{\alpha \in \mathbf{N}^{d}} c_{\alpha} e_{\alpha} ; \sup _{\alpha \in \mathbf{N}^{d}}\left(\left|c_{\alpha}\right| R^{-|\alpha|}\right)<\infty\right\}
$$

to each others, where $D_{d, R}$ is the polydisc

$$
\left\{\left(z_{1}, \ldots, z_{d}\right) \in \mathbf{C}^{d} ;\left|z_{j}\right| \leq R, j=1, \ldots, d\right\} .
$$

Theorem 3.10. Let $\Omega_{j, R}, j=0,1,2$, be given by (3.12) -(3.14) when $R>0$, and let $R_{0}, R \in \mathbf{R}$ be such that $0<R_{0}<R$. Then

$$
\Omega_{0, R} \subseteq \Omega_{2, R} \quad \text { and } \quad \Omega_{2, R_{0}} \subseteq \Omega_{1, R} .
$$

In particular,

$$
\mathcal{S}_{C}\left(\mathbf{R}^{d}\right)=\mathcal{H}_{b_{1}}\left(\mathbf{R}^{d}\right) \quad \text { and } \quad \mathcal{H}_{0, b_{1}}\left(\mathbf{R}^{d}\right) \subsetneq \mathcal{S}_{C}\left(\mathbf{R}^{d}\right) .
$$

We need some preparations for the proof, and begin with the following lemma. 
Lemma 3.11. Let $\chi$ be the characteristic function of the polydisc

$$
\left\{z=\left(z_{1}, \ldots, z_{d}\right) \in \mathbf{C}^{d} ;\left|z_{j}\right| \leq 1, j=1, \ldots, d\right\},
$$

and let

$$
F_{\alpha}(z)=\sqrt{\alpha !}\left(\prod_{j=1}^{d}\left(\alpha_{j}+1\right)\right) z^{\alpha} e^{|z|^{2}} \chi(z), \quad z \in \mathbf{C}^{d}, \alpha \in \mathbf{N}^{d} .
$$

Then

$$
\Pi_{A} F_{\alpha}=e_{\alpha}
$$

Proof. Since

$$
\Pi_{A} F_{\alpha}=\left(\Pi_{A} F_{\alpha_{1}}\right) \otimes \cdots \otimes\left(\Pi_{A} F_{\alpha_{d}}\right) \quad \text { and } \quad e_{\alpha}=e_{\alpha_{1}} \otimes \cdots \otimes e_{\alpha_{d}},
$$

when $\alpha=\left(\alpha_{1}, \ldots, \alpha_{d}\right) \in \mathbf{N}^{d}$, it suffices to prove the result for $d=1$. Furthermore, since both sides in (3.16) are entire, it suffices to prove

$$
\Pi_{A} F_{\alpha}\left(e^{i \varphi}\right)=e_{\alpha}\left(e^{i t}\right)=\sqrt{e^{i \alpha \varphi}} \sqrt{\alpha !}, \quad \varphi \in \mathbf{R},
$$

$(3.16)^{\prime}$

by analytic continuation.

By taking polar coordinates when integrating we get

$$
\begin{aligned}
\Pi_{A} F_{\alpha}\left(e^{i \varphi}\right)=\pi^{-1} \sqrt{\alpha !}(\alpha+1) \int_{|w| \leq 1} & w^{\alpha} e^{e^{i \varphi} \bar{w}} d \lambda(w) \\
& =\sqrt{\alpha !}(\alpha+1) \int_{0}^{1} r^{\alpha+1} I_{\alpha}(r, \varphi) d r
\end{aligned}
$$

where

$$
\begin{gathered}
I_{\alpha}(r, \varphi)=\pi^{-1} \int_{0}^{2 \pi} e^{i \alpha \theta} e^{r e^{\varphi-\theta}} d \theta \\
=\pi^{-1} e^{i \alpha \varphi} \int_{0}^{2 \pi} e^{-i \alpha \theta} e^{r e^{i \theta}} d \theta \\
=2 e^{i \alpha \varphi}\left(\frac{1}{2 \pi i} \int_{\gamma} \frac{e^{r z}}{z^{\alpha+1}} d z\right),
\end{gathered}
$$

where $\gamma$ is the unit circle in counterclockwise direction. Hence,

$$
\frac{1}{2 \pi i} \int_{\gamma} \frac{e^{r z}}{z^{\alpha+1}} d z=\left.\frac{1}{\alpha !} \frac{d^{\alpha}}{d z^{\alpha}}\left(e^{r z}\right)\right|_{z=0}=\frac{r^{\alpha}}{\alpha !}
$$

by Cauchy's integral formula.

This gives

and

$$
I_{\alpha}(r, \varphi)=\frac{2 e^{i \alpha \varphi} r^{\alpha}}{\alpha !}
$$

$$
\Pi_{A} F_{\alpha}\left(e^{i \varphi}\right)=\frac{2(\alpha+1) e^{i \alpha \varphi}}{\sqrt{\alpha !}} \int_{0}^{1} r^{2 \alpha+1} d r=\frac{e^{\alpha \varphi}}{\sqrt{\alpha !}}=e_{\alpha}\left(e^{i \varphi}\right),
$$

and the result follows. 
Proof of Theorem 3.10. By Proposition 3.3, the result follows if we prove (3.15). Assume that $F \in \Omega_{0, R}$. Then

$$
\left|F(w) e^{(z, w)}\right| \lesssim e^{R|z|} .
$$

This gives

which implies $\Omega_{0, R} \subseteq \Omega_{2, R}$.

$$
\left|\mathfrak{V}_{d} f(z)\right| \lesssim e^{R|z|} \int_{\mathbf{C}^{d}} d \mu(w) \asymp e^{R|z|},
$$

It remains to prove $\Omega_{2, R_{0}} \subseteq \Omega_{1, R}$. By

$$
\left(\Pi_{A} F\right)(R z)=\left(\Pi_{A} F_{R}\right)(z), \quad \text { when } \quad F_{R}(z)=R^{-2 d} e^{\left(1-\frac{1}{R^{2}}\right)|w|^{2}} F\left(\frac{z}{R}\right),
$$

we may reduce ourself to the case when $R=1$ and $R_{0}<1$.

Let $F_{\alpha}$ be as in the proof of Lemma 3.11. By the latter lemma and Weierstrass theorem it suffices to prove that

$$
\sum_{\alpha \in \mathbf{N}^{d}}\left|c_{\alpha}\right|\left\|F_{\alpha}\right\|_{L^{\infty}}<\infty
$$

since $\operatorname{supp}\left(F_{\alpha}\right) \subseteq D_{d, 1}$.

We have

$$
\sum_{\alpha \in \mathbf{N}^{d}}\left|c_{\alpha}\right|\left\|F_{\alpha}\right\|_{L^{\infty}} \lesssim \sum_{\alpha \in \mathbf{N}^{d}}\left(\prod_{j=1}^{d}\left(\alpha_{j}+1\right)\right) R_{1}^{|\alpha|}<\infty
$$

and the result follows.

Example 3.12. Let $F(z)=e^{|z|^{2}} \chi(z)$, where $\chi$ is the characteristic function of the set $[0.1]^{2 d}$, and let $f$ be chosen such that $\mathfrak{V}_{d} f=\Pi_{A} F$. Then $f \in \mathcal{S}_{C}\left(\mathbf{R}^{d}\right)$ since $F \in L^{\infty}\left(\mathbf{R}^{d}\right) \cap \mathscr{E}^{\prime}\left(\mathbf{R}^{d}\right)$.

On the other hand, by straight-forward computations one obtains

$$
\mathfrak{V}_{d} f(z)=\Pi_{A} F(z)=\pi^{-d}\left(\prod_{j=1}^{d} \frac{e^{z_{j}}-1}{z_{j}}\right)\left(\prod_{j=1}^{d} \frac{1-e^{-i z_{j}}}{i z_{j}}\right) .
$$

This implies that

$$
z \mapsto\left|\mathfrak{V}_{d} f(z)\right| e^{-\varepsilon|z|}
$$

is unbounded when $\varepsilon>0$ is small enough. Consequently, $f \notin \mathcal{H}_{0, b_{1}}\left(\mathbf{R}^{d}\right)$.

4. Characterizations of $\mathcal{H}_{s}\left(\mathbf{R}^{d}\right)$ And $\mathcal{H}_{0, s}\left(\mathbf{R}^{d}\right)$, And their

$$
\text { DUALS, FOR } s \in \mathbf{R}_{+}
$$

In this section we show that $\boldsymbol{\Sigma}_{s}, \mathcal{S}_{s}$ and their duals are equal to $\mathcal{H}_{0, s}$, $\mathcal{H}_{s}$ and their duals, respectively. We also describe their images under the Bargmann transform as convenient spaces of entire functions.

First we need certain invariance properties concerning the norm condition (1.6). More precisely, the following result links the conditions

$$
\sup _{N \geq 0} \frac{\left\|H^{N} f\right\|_{L^{p_{0}}}}{h^{N}(N !)^{2 s}}<\infty
$$


and

$$
\sup _{N \geq N_{0}} \frac{\left\|H^{N} f\right\|_{M_{(\omega)}^{p, q}}}{h^{N}(N !)^{2 s}}<\infty .
$$

to each others and shows in particular that the $L^{\infty}$ norm in (1.6) can be replaced by other types of Lebesgue or modulation space quasi-norms.

Proposition 4.1. Let $p_{0} \in[1, \infty], p, q \in(0, \infty], N_{0} \geq 0$ be an integer, $s \geq 0$ and let $\omega \in \mathscr{P}\left(\mathbf{R}^{2 d}\right)$. Then the following conditions are equivalent:

(1) (4.1) holds for some $h>0$ (for every $h>0$ );

(2) (4.2) holds for some $h>0$ (for every $h>0$ ).

Proof. First we prove that (4.2) is independent of $N_{0} \geq 0$ when $p, q \geq 1$. Evidently, if (4.2) is true for $N_{0}=0$, then it is true also for $N_{0}>0$. On the other hand, the map

$$
H^{N}: M_{\left(v_{N} \omega\right)}^{p, q}\left(\mathbf{R}^{d}\right) \rightarrow M_{(\omega)}^{p, q}\left(\mathbf{R}^{d}\right), \quad v_{N}(x, \xi)=\left(1+|x|^{2}+|\xi|^{2}\right)^{N}
$$

and its inverse are continuous and bijective (cf. e. g. [23, Theorem 3.10]). Hence, if $0 \leq N \leq N_{0}, N_{1}=N_{0}-N \geq 0$ and (4.2) holds for some $N_{0} \geq 0$, then

$$
\left\|H^{N} f\right\|_{M_{(\omega)}^{p, q}} \lesssim\left\|H^{N_{0}} f\right\|_{M_{\left(\omega / v_{N}\right)}^{p, q}} \lesssim\left\|H^{N_{0}} f\right\|_{M_{(\omega)}^{p, q}}<\infty
$$

and (4.2) holds for $N_{0}=0$. This implies that (4.2) is independent of $N_{0} \geq 0$ when $p, q \geq 1$.

Next we prove that (2) is independent of the choice of $\omega \in \mathscr{P}\left(\mathbf{R}^{2 d}\right)$. For every $\omega_{1}, \omega_{2} \in \mathscr{P}\left(\mathbf{R}^{2 d}\right)$, we may find an integer $N_{0} \geq 0$ such that

$$
\frac{1}{v_{N_{0}}} \lesssim \omega_{1}, \omega_{2} \lesssim v_{N_{0}}
$$

and then

$$
\|f\|_{M_{\left(1 / v_{N_{0}}\right)}^{p, q}} \lesssim\|f\|_{M_{\left(\omega_{1}\right)}^{p, q}},\|f\|_{M_{\left(\omega_{2}\right)}^{p, q}} \lesssim\|f\|_{M_{\left(v_{N_{0}}\right)}^{p, q}}
$$

Hence the stated invariance follows if we prove that (4.2) holds for $\omega=v_{N_{0}}$, if it is true for $\omega=1 / v_{N_{0}}$. 
Therefore, assume that (4.2) holds for $\omega=1 / v_{N_{0}}$. If $N \geq 2 N_{0}$, then the bijectivity of (4.3) gives

$$
\begin{aligned}
& \frac{\left\|H^{N} f\right\|_{M_{\left(v_{0}\right)}^{p, q}}}{h^{N}(N !)^{2 s}} \lesssim \frac{\left\|H^{N+2 N_{0}} f\right\|_{M_{\left(1 / v_{N_{0}}\right)}^{p, q}}}{h^{N}(N !)^{2 s}} \\
& \quad=h^{2 N_{0}}\left(\begin{array}{c}
N+2 N_{0} \\
2 N_{0}
\end{array}\right)^{2 s}\left(\left(2 N_{0}\right) !\right)^{2 s} \frac{\left\|H^{N+2 N_{0}} f\right\|_{M_{\left(1 / v_{N_{0}}\right)}^{p, q}}}{h^{N+2 N_{0}}\left(\left(N+2 N_{0}\right) !\right)^{2 s}} \\
& \asymp\left(\begin{array}{c}
N+2 N_{0} \\
2 N_{0}
\end{array}\right)^{2 s} \frac{\left\|H^{N+2 N_{0}} f\right\|_{\left.M_{\left(1 / v_{0}\right.}^{p, q}\right)} \frac{\left\|H^{N+2 N_{0}} f\right\|_{\left.M_{\left(1 / v_{0}\right.}^{p, q}\right)}}{h^{N+2 N_{0}}\left(\left(N+2 N_{0}\right) !\right)^{2 s}}}{\sim} \frac{h_{1}^{N+2 N_{0}}\left(\left(N+2 N_{0}\right) !\right)^{2 s}}{h^{2 s}}
\end{aligned}
$$

where $h_{1}=\frac{h}{4^{s}}$. This gives the invariance of (2) with respect to $\omega$ in the case $p, q \geq 1$. For general $p, q>0$, the invariance of (4.2) with respect to $\omega, p$ and $q$ is now a consequence of the embeddings

$$
M_{\left(v_{N} \omega\right)}^{\infty}\left(\mathbf{R}^{d}\right) \subseteq M_{(\omega)}^{p, q}\left(\mathbf{R}^{d}\right) \subseteq M_{(\omega)}^{\infty}\left(\mathbf{R}^{d}\right), \quad N>\frac{d}{p} .
$$

The equivalence between (1) and (2) follows from these invariance properties and the continuous embeddings

$$
M^{p_{0}, q_{1}} \subseteq L^{p_{0}} \subseteq M^{p_{0}, q_{2}}, \quad q_{1}=\min \left(p_{0}, p_{0}^{\prime}\right), \quad q_{2}=\max \left(p_{0}, p_{0}^{\prime}\right),
$$

which can be found in e.g. [25].

The next three theorems characterize spaces in (2.8) in terms of Pilipović spaces and spaces of entire functions. Here we let

$$
\omega_{1, r, s}(z)=\left\{\begin{array}{ll}
e^{r(\log \langle z\rangle) \frac{1}{1-2 s}}, & s<\frac{1}{2} \\
e^{\frac{|z|^{2}}{2}-r|z|^{\frac{1}{s}}}, & s \geq \frac{1}{2},
\end{array} \quad \text { and } \quad \omega_{2, r, s}(z)=e^{\frac{|z|^{2}}{2}+r|z|^{\frac{1}{s}}},\right.
$$

Theorem 4.2. Let $s \geq 0$. Then

$$
\begin{array}{rlrl}
\boldsymbol{\Sigma}_{s}\left(\mathbf{R}^{d}\right) & =\mathcal{H}_{0, s}\left(\mathbf{R}^{d}\right), & \mathcal{S}_{s}\left(\mathbf{R}^{d}\right) & =\mathcal{H}_{s}\left(\mathbf{R}^{d}\right), \\
\mathcal{S}_{s}^{\prime}\left(\mathbf{R}^{d}\right)=\mathcal{H}_{s}^{\prime}\left(\mathbf{R}^{d}\right), & \boldsymbol{\Sigma}_{s}^{\prime}\left(\mathbf{R}^{d}\right)=\mathcal{H}_{0, s}^{\prime}\left(\mathbf{R}^{d}\right) .
\end{array}
$$

Theorem 4.3. Let $s \geq 0$ and let $\omega_{1, r, s}$ be as in (4.5). Then the following is true:

(1) the map (2.14) extends uniquely to a homeomorphism from $\mathcal{A}_{s}\left(\mathbf{C}^{d}\right)$ to

$$
\left\{F \in A\left(\mathbf{C}^{d}\right) ;|F(z)| \lesssim \omega_{1, r, s} \text { for some } r>0\right\} ;
$$

(2) if in addition $s \notin\left\{0, \frac{1}{2}\right\}$, then the map (2.14) extends uniquely to a homeomorphism from $\mathcal{A}_{0, s}\left(\mathbf{C}^{d}\right)$ to

$$
\left\{F \in A\left(\mathbf{C}^{d}\right) ;|F(z)| \lesssim \omega_{1, r, s} \text { for every } r>0\right\} .
$$

Theorem 4.4. Let $s \geq \frac{1}{2}$ and let $\omega_{1, r, s}$ be as in (4.5). Then the following is true: 
(1) the map (2.14) extends uniquely to a homeomorphism from $\mathcal{A}_{s}^{\prime}\left(\mathbf{C}^{d}\right)$ to

$$
\left\{F \in A\left(\mathbf{C}^{d}\right) ;|F(z)| \lesssim \omega_{2, r, s} \text { for every } r>0\right\}
$$

(2) if in addition $s \neq \frac{1}{2}$, then the map (2.14) extends uniquely to a homeomorphism from $\mathcal{A}_{0, s}^{\prime}\left(\mathbf{C}^{d}\right)$ to

$$
\left\{F \in A\left(\mathbf{C}^{d}\right) ;|F(z)| \lesssim \omega_{2, r, s} \text { for some } r>0\right\} ;
$$

Again we recall that Remark 1.12 explains the topologies of the spaces in (4.7)-(4.10).

Remark 4.5. Evidently, since the Bargmann transform is bijective between the spaces in (2.8) and (2.9), Theorems 3.1, 3.2, 4.3, and 4.4 remains true if the spaces in (2.9) and the map (2.14) are replaced by corresponding spaces in (2.8) and the Bargmann transform, respectively.

Remark 4.6. Because of the strong links between the Bargmann transform and the short-time Fourier transform when the window function is given by $\phi$ in (1.14), Theorem 4.3 implies that the conditions on the right-hand sides of (3.2)-(3.5) and (4.7)-(4.9) carry over to analogous conditions on the short-time Fourier transforms. For example, (1) in Theorem 3.1 shows that $f \in \mathcal{H}_{0, \frac{1}{2}}\left(\mathbf{R}^{d}\right)$ is equivalent to

$$
\left|\left(V_{\phi} f\right)(x, \xi)\right| \lesssim e^{-\frac{1}{4}(1-\varepsilon)\left(|x|^{2}+|\xi|^{2}\right)} \quad \text { for every } \varepsilon>0 .
$$

In Section 6 we present a more comprehensive list of relations between elements in function spaces and estimates of their short-time Fourier transform (see Proposition 6.5).

Theorem 4.3 in the case $0<s<\frac{1}{2}$ follows by suitable applications of Theorem [2.5, and is presented in [9]. Moreover, proofs of extended versions of Theorems 4.3 and 4.4 in the case $s \geq \frac{1}{2}$ are given in Section 5. using the facts that $\boldsymbol{\Sigma}_{s}\left(\mathbf{R}^{d}\right)=\Sigma_{s}\left(\mathbf{R}^{d}\right)$ when $s>\frac{1}{2}$ and $\mathcal{S}_{s}\left(\mathbf{R}^{d}\right)=$ $\mathcal{S}_{s}\left(\mathbf{R}^{d}\right)$ when $s \geq \frac{1}{2}$. (See Theorem 5.2 and its proof.)

At this moment it therefore remains to prove Theorem 4.2, and then the following lemma takes care of the case $s>0$.

Lemma 4.7. Let $C, s, h>0, f \in \mathcal{H}_{0}^{\prime}\left(\mathbf{R}^{d}\right)$, and let $c_{\alpha}(f)$ be the same as in (2.10). Then the following is true:

(1) if

$$
\left\|H^{N} f\right\|_{L^{2}} \leq C h^{N}(N !)^{2 s}
$$

for every integer $N \geq 0$, then

$$
\left|c_{\alpha}(f)\right| \leq 4^{s} C e^{-s|\alpha|^{\frac{1}{2 s}} / h^{\frac{1}{2 s}}} ;
$$


(2) if

$$
\left|c_{\alpha}(f)\right| \leq C e^{-\frac{1}{h}|\alpha|^{\frac{1}{2 s}}}
$$

then

$$
\left\|H^{N} f\right\|_{L^{2}} \leq C_{1}\left(1+2 s h^{2 s d} \Gamma(2 s d)\right)\left(3(4 s h)^{2 s}\right)^{N}(N !)^{2 s},
$$

for some constant $C_{1}$ which only depends on $C$ and $d$.

Proof. Assume that (4.11) holds for every $N \geq 0$. Since $H^{N} h_{\alpha}=(2|\alpha|+$ $d)^{N} h_{\alpha}$ and $\left\{h_{\alpha}\right\}_{\alpha \in \mathbf{N}^{d}}$ is an orthonormal basis for $L^{2}$, we get

$$
(2|\alpha|+d)^{N}\left|c_{\alpha}(f)\right| \leq\left\|H^{N} f\right\|_{L^{2}} \leq C h^{N}(N !)^{2 s} .
$$

Hence,

which implies

$$
\left|c_{\alpha}(f)\right| \leq \frac{C h^{N}(N !)^{2 s}}{(2|\alpha|+d)^{N}}
$$

$$
\left|c_{0}(f)\right| \leq C h^{N}(N !)^{2 s} \quad \text { and } \quad\left|c_{\alpha}(f)\right| \leq \frac{C h^{N}(N !)^{2 s}}{|\alpha|^{N}}, \quad|\alpha| \geq 1 .
$$

In particular, (4.12) holds when $\alpha=0$.

We may therefore assume that $|\alpha| \geq 1$. By (4.15) we get

$$
\left|c_{\alpha}(f)\right|^{\frac{1}{2 s}} \cdot \frac{\left(\frac{|\alpha|^{\frac{1}{2 s}}}{2 h^{\frac{1}{2 s}}}\right)^{N}}{N !} \leq C^{\frac{1}{2 s}} \frac{1}{2^{N}}
$$

and by summing over all $N \geq 0$ it follows that

$$
\left|c_{\alpha}(f)\right|^{\frac{1}{2 s}} e^{|\alpha|^{\frac{1}{2 s}} /\left(2 h^{\frac{1}{2 s}}\right)} \leq 2 C^{\frac{1}{2 s}},
$$

which is the same as (4.12). This gives (1).

Next assume that (4.13) holds. Then Parseval's formula gives

$$
\begin{aligned}
\left\|H^{N} f\right\|_{L^{2}} \lesssim & \left(\sum_{\alpha}(2|\alpha|+d)^{2 N} e^{-\frac{2}{h}|\alpha|^{\frac{1}{2 s}}}\right)^{\frac{1}{2}} \\
& \lesssim 1+\left(\sum_{|\alpha| \geq 1} 9^{N}|\alpha|^{2 N} e^{-\frac{2}{h}|\alpha|^{\frac{1}{2 s}}}\right)^{\frac{1}{2}} \lesssim 1+3^{N} R \sup _{t \geq 1} g(t),
\end{aligned}
$$

where

$$
R=\sum_{k=1}^{\infty} k^{d-1} e^{-k^{\frac{1}{2 s}} / h} \quad \text { and } \quad g(t)=t^{N} e^{-t^{\frac{1}{2 s}} /(2 h)} .
$$

We need to estimate $R$ and the last supremum. We have

$$
\begin{aligned}
R^{2} \lesssim 1+\int_{1}^{\infty} r^{d-1} e^{-r^{\frac{1}{2 s}} / h} d r=1+2 s h^{2 s d} \int_{1 /(2 h)}^{\infty} & e^{-r} r^{2 s d-1} d r \\
& \leq 1+2 s h^{2 s d} \Gamma(2 s d) .
\end{aligned}
$$


Furthermore, by straight-forward computations, it follows that $g(t)$ for $t \geq 1$ attains its maximum when $t=(4 s h N)^{2 s}$. Hence, by Stirling's formula we get

$$
\begin{aligned}
& \sup _{t \geq 1} g(t)=g\left((4 s h N)^{2 s}\right)=\left((4 s h)^{2 s}\right)^{N}\left(\frac{N^{N}}{e^{N}}\right)^{2 s} \\
& \lesssim\left((4 s h)^{2 s}\right)^{N}(2 \pi N)^{-s}(N !)^{2 s} \lesssim\left((4 s h)^{2 s}\right)^{N}(N !)^{2 s} .
\end{aligned}
$$

The result now follows by combining these estimates.

Proof of Theorem 4.2. It remains to prove (4.6) in the case $s=0$.

Assume that $f \in \mathcal{S}_{0}\left(\mathbf{R}^{d}\right)$. By Proposition 4.1 it follows that $\left\|H^{N} f\right\|_{L^{2}} \leq$ $C h^{N}$ for some $h>0$ and $C>0$ which are independent of $N \geq 0$. If $c_{\alpha}(f)$ is the same as in (2.10), then

$$
\sum_{\alpha \in \mathbf{N}^{d}}\left|(2|\alpha|+d)^{N} c_{\alpha}(f)\right|^{2}=\left\|H^{N} f\right\|_{L^{2}}^{2} \leq C^{2} h^{2 N},
$$

giving that

$$
\left|c_{\alpha}(f)\right| \leq C h^{N} \mid(2|\alpha|+d)^{-N} .
$$

If $\alpha$ is chosen such that $h<2|\alpha|+d$, then it follows by letting $N$ tends to infinity at the last estimate that $c_{\alpha}(f)=0$. This shows that $f \in \mathcal{H}_{0}\left(\mathbf{R}^{d}\right)$ and hence $\mathcal{S}_{0}\left(\mathbf{R}^{d}\right) \subseteq \mathcal{H}_{0}\left(\mathbf{R}^{d}\right)$.

On the other hand, for any Hermite function $h_{\alpha}$ we have

$$
\left\|H^{N} h_{\alpha}\right\|_{L^{2}}=(2|\alpha|+d)^{N}\left\|h_{\alpha}\right\|_{L^{2}} \leq h^{N}
$$

when $2|\alpha|+d \leq h$. Hence any Hermite function belongs to $\mathcal{S}_{0}\left(\mathbf{R}^{d}\right)$, and it follows that $\mathcal{H}_{0}\left(\mathbf{R}^{d}\right) \subseteq \mathcal{S}_{0}\left(\mathbf{R}^{d}\right)$. This gives (4.6).

By a straight-forward combination of the (4.2), Theorems 3.3, 3.4, 4.3 and 4.4, and general embedding relations for Gelfand-Shilov spaces, we get the following result.

Theorem 4.8. Let $s_{1}, s_{2} \in \mathbf{R}_{b}$ be such that $s_{1}<s_{2}$. Then the embeddings in (0.10) and (0.11) hold true, and are continuous and dense. Furthermore, the embeddings

$$
\Sigma_{s_{2}}^{\prime}\left(\mathbf{R}^{d}\right) \subseteq \mathcal{S}_{s_{1}}^{\prime}\left(\mathbf{R}^{d}\right) \subseteq \Sigma_{s_{1}}^{\prime}\left(\mathbf{R}^{d}\right) \subseteq \mathcal{S}_{0}^{\prime}\left(\mathbf{R}^{d}\right)
$$

hold true and are continuous.

4.1. Some consequences. Next we show some consequences of the previous results. As an immediate consequence of Theorem 4.3 we have

$$
\begin{aligned}
\Sigma_{s}\left(\mathbf{R}^{d}\right)=\bigcap_{r>0} \mathcal{H}_{\left[\vartheta_{r}\right]}^{2}\left(\mathbf{R}^{d}\right) \quad \text { and } \quad \mathcal{S}_{s}\left(\mathbf{R}^{d}\right)=\bigcup_{r>0} \mathcal{H}_{\left[\vartheta_{r}\right]}^{2}\left(\mathbf{R}^{d}\right), \\
\text { when } \vartheta_{r}(\alpha)=e^{r|\alpha|^{\frac{1}{2 s}}}, \quad s>0 \quad \text { and } \quad p \in(0, \infty]
\end{aligned}
$$

(see also [21,22]). 
We may also apply Theorem 4.3 and its proof to characterize $\boldsymbol{\Sigma}\left(\mathbf{R}^{d}\right)$ and $\boldsymbol{\Sigma}^{\prime}\left(\mathbf{R}^{d}\right)$ in terms of modulation spaces. In fact, by Proposition 2.8 and Theorem 4.3 it follows that $\boldsymbol{\Sigma}\left(\mathbf{R}^{d}\right)$ and $\boldsymbol{\Sigma}^{\prime}\left(\mathbf{R}^{d}\right)$ are the projective and inductive limit, respectively, of

$$
M_{\left(\omega_{h}\right)}^{2}\left(\mathbf{R}^{d}\right), \quad \omega_{h}(x, \xi)=e^{\frac{1}{4}(1-2 h)\left(|x|^{2}+|\xi|^{2}\right)}
$$

with respect to $h>0$. In particular,

$$
\begin{gathered}
\Sigma\left(\mathbf{R}^{d}\right)=\bigcap_{h>0} M_{\left(\omega_{h}\right)}^{2}\left(\mathbf{R}^{d}\right) \quad \text { and } \quad \Sigma^{\prime}\left(\mathbf{R}^{d}\right)=\bigcup_{h>0} M_{\left(\omega_{h}\right)}^{2}\left(\mathbf{R}^{d}\right), \\
\omega_{h}(x, \xi)=e^{\frac{1}{4}(1-2 h)\left(|x|^{2}+|\xi|^{2}\right)} .
\end{gathered}
$$

Let $\mathscr{B}$ be an invariant quasi-norm space and let $\sigma_{r}(x, \xi)=\langle x\rangle^{r}\langle\xi\rangle^{r}$. Since

$$
\Omega=\left\{\omega_{\varepsilon} \cdot \sigma_{r} ; r \in \mathbf{R}\right\}
$$

is an admissible family of weights, a combination of [26, Theorem 3.2], Theorem 3.8 and (4.17) shows that (4.17) can be extended into

$$
\begin{gathered}
\Sigma\left(\mathbf{R}^{d}\right)=\bigcap_{h>0} M\left(\omega_{h}, \mathscr{B}\right) \quad \text { and } \quad \Sigma^{\prime}\left(\mathbf{R}^{d}\right)=\bigcup_{h>0} M\left(\omega_{h}, \mathscr{B}\right), \\
\omega_{h}(x, \xi)=e^{\frac{1}{4}(1-2 h)\left(|x|^{2}+|\xi|^{2}\right)} .
\end{gathered}
$$

Furthermore, since for any weight $\omega \in \mathscr{P}_{Q}\left(\mathbf{R}^{2 d}\right)$ we have $\omega_{h} \lesssim \omega$, for some $\varepsilon>0$, (4.17) $)^{\prime}$ gives

$$
\Sigma^{\prime}\left(\mathbf{R}^{d}\right)=\bigcup_{\omega \in \mathscr{P}_{Q}\left(\mathbf{R}^{2 d}\right)} M(\omega, \mathscr{B}) .
$$

An other consequence of Theorem 4.3 is the following.

Proposition 4.9. Let $F(z)=e^{\gamma z^{2}}$, where $\gamma \in \mathbf{C}$. Then $F=\mathfrak{V}_{d} f$ for some $f \in \Sigma^{\prime}\left(\mathbf{R}^{d}\right)$.

\section{MAPPing PROPERTiES OF GELFAND-Shilov SPACES AND THEIR DISTRIBUTION SPACES, UNDER THE BARGMANN TRANSFORM}

In this section we discuss the image of the Bargmann transform on Gelfand-Shilov spaces and their distribution spaces. We also use the results to show that the Gelfand-Shilov spaces of functions or distributions can be obtained by appropriate unions or intersections of certain modulation spaces, introduced in [26].

With inspiration of the links between the spaces in (2.8), (2.9), the Pilipović spaces and Gelfand-Shilov spaces, we make the following definition.

Definition 5.1. Let $s_{1}, t_{1} \geq \frac{1}{2}$ and $s_{2}, t_{2}>\frac{1}{2}$, and let

$$
\omega_{r, s, t}(z)=e^{\frac{|z|^{2}}{2}+r\left(|x|^{\frac{1}{t}}+|\xi|^{\frac{1}{s}}\right)}, \quad z=x+i \xi, x, \xi \in \mathbf{R}^{d},
$$


when $s, t \geq \frac{1}{2}$. Then $\mathcal{A}_{t_{1}}^{s_{1}}\left(\mathbf{C}^{d}\right), \mathcal{A}_{0, t_{2}}^{s_{2}}\left(\mathbf{C}^{d}\right),\left(\mathcal{A}_{0, t_{2}}^{s_{2}}\right)^{\prime}\left(\mathbf{C}^{d}\right)$ and $\left(\mathcal{A}_{t_{1}}^{s_{1}}\right)^{\prime}\left(\mathbf{C}^{d}\right)$ are given by

$$
\begin{aligned}
\mathcal{A}_{t_{1}}^{s_{1}}\left(\mathbf{C}^{d}\right) & =\left\{F \in A\left(\mathbf{C}^{d}\right) ;|F(z)| \lesssim \omega_{r, s_{1}, t_{1}}(z) \text { for some } r<0\right\} \\
\mathcal{A}_{0, t_{2}}^{s_{2}}\left(\mathbf{C}^{d}\right) & =\left\{F \in A\left(\mathbf{C}^{d}\right) ;|F(z)| \lesssim \omega_{r, s_{2}, t_{2}}(z) \text { for every } r<0\right\} \\
\left(\mathcal{A}_{0, t_{2}}^{s_{2}}\right)^{\prime}\left(\mathbf{C}^{d}\right) & =\left\{F \in A\left(\mathbf{C}^{d}\right) ;|F(z)| \lesssim \omega_{r, s_{2}, t_{2}}(z) \text { for some } r>0\right\} \\
\left(\mathcal{A}_{t_{1}}^{s_{1}}\right)^{\prime}\left(\mathbf{C}^{d}\right) & =\left\{F \in A\left(\mathbf{C}^{d}\right) ;|F(z)| \lesssim \omega_{r, s_{1}, t_{1}}(z) \text { for every } r>0\right\},
\end{aligned}
$$

We also set $\mathcal{A}_{s}=\mathcal{A}_{s}^{s}$ and $\mathcal{A}_{0, s}=\mathcal{A}_{0, s}^{s}$, and remark that $\mathcal{A}_{s}=\mathcal{A}_{s}$ when $s \geq \frac{1}{2}$ and $\mathcal{A}_{0, s}=\mathcal{A}_{0, s}$ when $s>\frac{1}{2}$, since $\mathcal{S}_{s}\left(\mathbf{R}^{d}\right)=\mathcal{S}_{s}\left(\mathbf{R}^{d}\right)$ when $s \geq \frac{1}{2}$ and $\Sigma_{s}\left(\mathbf{R}^{d}\right)=\Sigma_{s}\left(\mathbf{R}^{d}\right)$ when $s>\frac{1}{2}$.

We note that if $p \in(0, \infty]$, then

$$
\begin{array}{rlrl}
\mathcal{A}_{t_{1}}^{s_{1}}\left(\mathbf{C}^{d}\right) & =\bigcup_{r>0} A_{\left(\vartheta_{r, s_{1}, t_{1}}\right)}^{p}\left(\mathbf{C}^{d}\right), & \mathcal{A}_{0, t_{2}}^{s_{2}}\left(\mathbf{C}^{d}\right) & =\bigcap_{r>0} A_{\left(\vartheta_{\left.r, s_{2}, t_{2}\right)}^{p}\left(\mathbf{C}^{d}\right),\right.}^{p} \\
\left(\mathcal{A}_{0, t_{2}}^{s_{2}}\right)^{\prime}\left(\mathbf{C}^{d}\right) & =\bigcup_{r>0} A_{\left(1 / \vartheta_{\left.r, s_{2}, t_{2}\right)}^{p}\right)}^{p}\left(\mathbf{C}^{d}\right) \quad \text { and } \quad\left(\mathcal{A}_{t_{1}}^{s_{1}}\right)^{\prime}\left(\mathbf{C}^{d}\right)=\bigcap_{r>0} A_{\left(1 / \vartheta_{r, s_{1}, t_{1}}\right)}^{p}\left(\mathbf{C}^{d}\right),
\end{array}
$$

in view of [26, Theorem 3.2]. Here $\vartheta_{r, s, t}$ is given by (1.27). We let the topologies of $\mathcal{A}_{t}^{s}\left(\mathbf{C}^{d}\right)$ and $\mathcal{A}_{0, t}^{s}\left(\mathbf{C}^{d}\right)$ be the inductive and projective limit topology, respectively, of $A_{\left(\vartheta_{r, s, t}\right)}^{p}\left(\mathbf{C}^{d}\right), r>0$, and the topologies of $\left(\mathcal{A}_{0, t}^{s}\right)^{\prime}\left(\mathbf{C}^{d}\right)$ and $\left(\mathcal{A}_{t}^{s}\right)^{\prime}\left(\mathbf{C}^{d}\right)$ be the inductive and projective limit topology, respectively, of $A_{\left(1 / \vartheta_{r, s, t}\right)}^{p}\left(\mathbf{C}^{d}\right), r>0$.

Theorem 5.2. Let $s_{1}, t_{1} \geq \frac{1}{2}, s_{2}, t_{2}>\frac{1}{2}$, and let $\omega_{r, s, t}$ be as in (5.1). Then the Bargmann transform from $\mathcal{H}_{0}\left(\mathbf{R}^{d}\right)$ to $A_{1 / 2}^{\prime}\left(\mathbf{C}^{d}\right)$ extends uniquely to homeomorphisms from

$$
\mathcal{S}_{t_{1}}^{s_{1}}\left(\mathbf{R}^{d}\right), \quad \Sigma_{t_{2}}^{s_{2}}\left(\mathbf{R}^{d}\right), \quad\left(\Sigma_{t_{2}}^{s_{2}}\right)^{\prime}\left(\mathbf{R}^{d}\right) \quad \text { and } \quad\left(\mathcal{S}_{t_{1}}^{s_{1}}\right)^{\prime}\left(\mathbf{R}^{d}\right)
$$

to

$$
\mathcal{A}_{t_{1}}^{s_{1}}\left(\mathbf{R}^{d}\right), \quad \mathcal{A}_{0, t_{2}}^{s_{2}}\left(\mathbf{R}^{d}\right), \quad\left(\mathcal{A}_{0, t_{2}}^{s_{2}}\right)^{\prime}\left(\mathbf{R}^{d}\right) \quad \text { and } \quad\left(\mathcal{A}_{t_{1}}^{s_{1}}\right)^{\prime}\left(\mathbf{R}^{d}\right),
$$

respectively.

Proof. Let $\phi$ be given by (1.14). By Propositions 1.1 and 1.2, and (1.24) it follows that the Bargmann transform maps the spaces in (5.3) into corresponding spaces in (5.4). Furthermore, these mappings are homeomorphisms from the spaces in (5.3) to corresponding images under the relative topologies induced from the spaces in (5.4). We need to prove that these images agree with the spaces in (5.4).

Therefore, let $F \in\left(\mathcal{A}_{t_{1}}^{s_{1}}\right)^{\prime}\left(\mathbf{C}^{d}\right)$. Since $\left(\mathcal{A}_{t_{1}}^{s_{1}}\right)^{\prime}\left(\mathbf{C}^{d}\right) \subseteq \mathcal{A}_{1 / 2}^{\prime}\left(\mathbf{C}^{d}\right)$, Theorems 4.2 and 4.4, and (1.7) shows that $F=\mathfrak{V}_{d} f$ for some $f \in \mathcal{S}_{1 / 2}^{\prime}\left(\mathbf{R}^{d}\right)$, and since $\phi \in \mathcal{S}_{t}^{s}\left(\mathbf{R}^{d}\right)$ for every $s, t \geq \frac{1}{2}$, (1.25) shows that (1.5) holds 
with $s_{1}$ and $t_{1}$ in place of $s$ and $t$, respectively, for every $r>0$. Hence, $f \in\left(\mathcal{S}_{t_{1}}^{s_{1}}\right)^{\prime}\left(\mathbf{R}^{d}\right)$, by Proposition 1.2.

In the same way, it is proved that the images of the other spaces in (5.3) agree with corresponding spaces in (5.4), and the result follows.

Remark 5.3. We remark here that already in [2], complete characterizations of the $\mathscr{S}\left(\mathbf{R}^{d}\right)$ and $\mathscr{S}^{\prime}\left(\mathbf{R}^{d}\right)$ in terms of the images under the Bargmann transform, are obtained. In fact, it is here proved that $\mathfrak{V}_{d}$ is bijective from $\mathscr{S}\left(\mathbf{R}^{d}\right)$ to

$$
\mathcal{A}_{\infty}\left(\mathbf{C}^{d}\right)=\left\{F \in A\left(\mathbf{C}^{d}\right) ;|F(z)| \lesssim e^{\frac{|z|^{2}}{2}}\langle z\rangle^{-N} \text { for every } N \geq 0\right\},
$$

and from $\mathscr{S}^{\prime}\left(\mathbf{R}^{d}\right)$ to

$$
\mathcal{A}_{\infty}\left(\mathbf{C}^{d}\right)=\left\{F \in A\left(\mathbf{C}^{d}\right) ;|F(z)| \lesssim e^{\frac{|z|^{2}}{2}}\langle z\rangle^{N} \text { for some } N \geq 0\right\} .
$$

Remark 5.4. Especially the cases when $s=s_{1}=\frac{1}{2}$ or $t=t_{1}=\frac{1}{2}$ in Theorem 5.2 seem to be new, and are often not taken into account in e. g. [26]. In fact, in [26] it is usually assumed that the involved weights should belong to $\mathscr{P}_{G}^{0}$ or the larger class $\mathscr{P}_{Q}^{0}$.

By relaxing the condition

$$
e^{-r|x|^{2}} \lesssim \omega(x) \lesssim e^{r|x|^{2}}, \quad \text { for every } r>0,
$$

into

$$
e^{-r|x|^{2}} \lesssim \omega(x) \lesssim e^{r_{0}|x|^{2}}, \quad \text { for every } r>0 \text { and some } r_{0}>0,
$$

in the definitions of $\mathscr{P}_{Q}^{0}\left(\mathbf{R}^{d}\right)$ and its subclasses, it follows that the results in [26], except Theorem 4.7 and Lemma 4.11, still hold in these more general situations.

We finish the section by the following extension of Theorem 3.9 in [26], and which follows from Theorems 3.2 and 3.4 in [26], and Theorem Proposition 1.2. Here recall for $\phi$ as in (1.14),$\omega$ a weight on $\mathbf{R}^{2 d}$ and $\mathscr{B}$ a mixed quasi-norm space on $\mathbf{R}^{2 d}, M(\omega, \mathscr{B})$ is the set of all $f \in \mathcal{S}_{1 / 2}^{\prime}\left(\mathbf{R}^{d}\right)$ such that $\|f\|_{M(\omega, \mathscr{B})} \equiv\left\|V_{\phi} f \cdot \omega\right\|_{\mathscr{B}}$ is finite.

Proposition 5.5. Let $\mathscr{B}$ be a mixed quasi-norm space on $\mathbf{R}^{2 d}$, and set

$$
\omega_{r}(x, \xi) \equiv e^{r\left(|x|^{\frac{1}{t}}+|\xi|^{\frac{1}{s}}\right)}, \quad r \in \mathbf{R} .
$$

Then the following is true:

(1) if $s, t \geq \frac{1}{2}$ then

$$
\bigcup_{r>0} M\left(\omega_{r}, \mathscr{B}\right)=\mathcal{S}_{t}^{s}\left(\mathbf{R}^{d}\right) \quad \text { and } \bigcap_{r<0} M\left(\omega_{r}, \mathscr{B}\right)=\left(\mathcal{S}_{t}^{s}\right)^{\prime}\left(\mathbf{R}^{d}\right) ;
$$

(2) if $s, t>\frac{1}{2}$, then

$$
\bigcap_{r>0} M\left(\omega_{r}, \mathscr{B}\right)=\Sigma_{t}^{s}\left(\mathbf{R}^{d}\right) \quad \text { and } \bigcup_{r<0} M\left(\omega_{r}, \mathscr{B}\right)=\left(\Sigma_{t}^{s}\right)^{\prime}\left(\mathbf{R}^{d}\right) .
$$




\section{Some CONSEQUENCES AND FURTHER REMARKS}

In this section we obtain further properties of the spaces in previous sections. First we deduce invariance properties under fractional Fourier transforms. Thereafter we obtain invariance properties under actions of linear partial differential operators with polynomial coefficients. Then we show some ideas on how to define spaces like $\boldsymbol{\Sigma}_{t}^{s}\left(\mathbf{R}^{d}\right), \mathcal{S}_{t}^{s}\left(\mathbf{R}^{d}\right)$ and their duals, and in the end we summarize the boundedness properties of the spaces in tables.

6.1. Some remarks on fractional Fourier transforms. Next we use the previous results to deduce some invariance properties for fractional Fourier transforms of element in certain modulation spaces.

If $f \in L^{2}\left(\mathbf{R}^{d}\right)$, then it is proved already in [1] that

$$
\left(\mathfrak{V}_{d}(\mathscr{F} f)\right)(z)=\left(\mathfrak{V}_{d} f\right)(-i z)=\left(\mathfrak{V}_{d} f\right)\left(e^{-i \frac{\pi}{2}} z\right) .
$$

The same arguments show that the latter equality still holds after the assumption $f \in L^{2}\left(\mathbf{R}^{d}\right)$ is relaxed into $f \in \mathcal{H}_{0}^{\prime}\left(\mathbf{R}^{d}\right)$. For this reason, the fractional Fourier transform, $\mathscr{F}_{r} f$ of $f$ of order $r \in \mathbf{R}$, is defined by the formula

$$
\left(\mathfrak{V}_{d}\left(\mathscr{F}_{r} f\right)\right)(z)=\left(\mathfrak{V}_{d} f\right)\left(e^{-i r \frac{\pi}{2}} z\right), \quad z \in \mathbf{C}^{d}
$$

(cf. Section 3b in 1], or [28] and the references therein). More generally, the partial fractional Fourier transform, $\mathscr{F}_{\boldsymbol{r}} f$ of $f$ of order $\boldsymbol{r}=$ $\left(r_{1}, \ldots, r_{d}\right) \in \mathbf{R}^{d}$ is defined by the formula

$$
\left(\mathfrak{V}_{d}\left(\mathscr{F}_{r} f\right)\right)(z)=\left(\mathfrak{V}_{d} f\right)\left(e^{-i r_{1} \frac{\pi}{2}} z_{1}, \ldots, e^{-i r_{d} \frac{\pi}{2}} z_{d}\right), \quad z \in \mathbf{C}^{d} .
$$

or equivalently,

$$
\begin{gathered}
\mathscr{F}_{\boldsymbol{r}} \equiv \mathfrak{V}_{d}^{-1} \circ T_{\boldsymbol{r}} \circ \mathfrak{V}_{d}, \\
\text { where } \quad\left(T_{\boldsymbol{r}} F\right)(z) \equiv F\left(e^{-i r_{1} \frac{\pi}{2}} z_{1}, \ldots, e^{-i r_{d} \frac{\pi}{2}} z_{d}\right)
\end{gathered}
$$

when $F \in \mathcal{A}_{0}^{\prime}\left(\mathbf{C}^{d}\right)$.

Certain parts of (1) and (2) in the following proposition are wellknown (cf. [1,2]). In order to be self-contained we here present a proof.

Proposition 6.1. Let $s \in \overline{\mathbf{R}_{b}}, \boldsymbol{r} \in \mathbf{R}^{d}, p \in(0, \infty]$ and let $\omega$ be a weight on $\mathbf{R}^{2 d}$ such that

$$
\omega(x, \xi)=\omega_{0}\left(\left|z_{1}\right|, \ldots,\left|z_{d}\right|\right), \quad z_{j}=x_{j}+i \xi_{j} \in \mathbf{C}, j=1, \ldots, d,
$$

for some $\omega_{0}$. Then the following is true:

(1) $\mathscr{F}_{\boldsymbol{r}}$ is continuous and bijective on $\mathcal{H}_{s}\left(\mathbf{R}^{d}\right), \mathcal{H}_{0, s}\left(\mathbf{R}^{d}\right)$ and $\mathscr{S}\left(\mathbf{R}^{d}\right)$, and their duals;

(2) $\mathscr{F}_{\boldsymbol{r}} h_{\alpha}=e^{-i\langle\boldsymbol{r}, \alpha\rangle \frac{\pi}{2}} h_{\alpha}$ for every $\alpha \in \mathbf{N}^{d}$, and $\mathscr{F}_{\boldsymbol{r}} \circ H=H \circ \mathscr{F}_{\boldsymbol{r}}$ on $\mathcal{S}_{0}^{\prime}\left(\mathbf{R}^{d}\right)$; 
(3) $\mathscr{F}_{\boldsymbol{r}}$ is isometric and bijective on $M_{(\omega)}^{p}\left(\mathbf{R}^{d}\right)$. Moreover, if $N$ is an integer and $f \in \mathcal{H}_{b_{1}}^{\prime}\left(\mathbf{R}^{d}\right)$, then

$$
\left\|H^{N}\left(\mathscr{F}_{r} f\right)\right\|_{M_{(\omega)}^{p}}=\left\|H^{N} f\right\|_{M_{(\omega)}^{p}} .
$$

Proof. By (1.20) and (6.1) we get

$$
\left(\mathfrak{V}_{d}\left(\mathscr{F}_{r} h_{\alpha}\right)\right)(z)=\frac{e^{-i\langle\alpha, \boldsymbol{r}\rangle \frac{\pi}{2}} z^{\alpha}}{(\alpha !)^{\frac{1}{2}}}=e^{-i\langle\alpha, \boldsymbol{r}\rangle \frac{\pi}{2}}\left(\mathfrak{V}_{d} h_{\alpha}\right)(z),
$$

which gives $\mathscr{F}_{\boldsymbol{r}} h_{\alpha}=e^{-i\langle\boldsymbol{r}, \alpha\rangle \frac{\pi}{2}} h_{\alpha}$ (see also [1]).

The assertion (1) now follows from the latter equality, Theorems 3.3 , 3.4 and 4.3 , Theorem 4.3 and duality.

Let $T_{\boldsymbol{r}}$ be as in (6.1), $f \in \mathcal{H}_{b_{1}}^{\prime}\left(\mathbf{R}^{d}\right)$, and let $F=\mathfrak{V}_{d} f$. By applying the Bargmann transform on $H\left(\mathscr{F}_{r} f\right)$ we get

$$
\begin{aligned}
\mathfrak{V}_{d}\left(H\left(\mathscr{F}_{\boldsymbol{r}} f\right)\right)(z) & =\sum_{j=1}^{d}\left(2 z_{j} \partial_{j}\left(T_{\boldsymbol{r}} F\right)(z)+d\left(T_{\boldsymbol{r}} F\right)(z)\right) \\
& =\sum_{j=1}^{d}\left(T_{\boldsymbol{r}}\left(2 z_{j} \partial_{j} F\right)(z)+d\left(T_{\boldsymbol{r}} F\right)(z)\right) \\
& =T_{\boldsymbol{r}}\left(\left(\sum_{j=1}^{d}\left(2 z_{j} \partial_{j}+d\right)\right) F\right)(z)=\mathfrak{V}_{d}\left(\mathscr{F}_{\boldsymbol{r}}(H f)\right)(z),
\end{aligned}
$$

and (2) follows.

We only prove (3) in the case $p<\infty$. The case $p=\infty$ follows by similar arguments and is left for the reader.

By identifying $(x, \xi) \in \mathbf{R}^{2 d}$ with $x+i \xi \in \mathbf{C}^{d}$, it follows from the assumptions that $\left(T_{r}^{-1} \omega\right)=\omega$. This gives

$$
\begin{aligned}
\left\|\mathscr{F}_{\boldsymbol{r}} f\right\|_{M_{(\omega)}^{p}}^{p} & =\left\|\mathfrak{V}_{d}\left(\mathscr{F}_{\boldsymbol{r}} f\right)\right\|_{A_{(\omega)}^{p}}^{p} \\
= & 2^{d}(2 \pi)^{-p \frac{d}{2}} \int_{\mathbf{C}^{d}}\left|\left(T_{\boldsymbol{r}} F\right)(z) e^{-\frac{|z|^{2}}{2}} \omega(\sqrt{2} \bar{z})\right|^{p} d \lambda(z) \\
= & 2^{d}(2 \pi)^{-p \frac{d}{2}} \int_{\mathbf{C}^{d}}\left|F(z) e^{-\frac{|z|^{2}}{2}} \omega(\sqrt{2} \bar{z})\right|^{p} d \lambda(z) \\
& =\left\|\mathfrak{V}_{d}(f)\right\|_{A_{(\omega)}^{p}}^{p}=\|f\|_{M_{(\omega)}^{p}}^{p},
\end{aligned}
$$

and (3) follows. The proof is complete.

6.2. Distribution theory properties. Next we discuss distribution theory properties of Pilipović spaces and their duals, and start with the following.

Proposition 6.2. Let $a(x, \xi)$ be a polynomial on $\mathbf{R}^{2 d}$ and let $s \in \overline{\mathbf{R}_{b}}$. Then the following is true: 
(1) the operator $a(x, D)$ on $\mathscr{S}\left(\mathbf{R}^{d}\right)$ is uniquely extendable to a continuous operator on $\mathcal{H}_{0}^{\prime}\left(\mathbf{R}^{d}\right)$;

(2) the operator $a(x, D)$ on $\mathcal{H}_{0}^{\prime}\left(\mathbf{R}^{d}\right)$ restricts to a continuous map on any of the spaces $\mathcal{H}_{0, s}\left(\mathbf{R}^{d}\right)$ and $\mathcal{H}_{s}\left(\mathbf{R}^{d}\right)$, and their duals.

Proof. Assume that $f$ belongs to

$$
\mathcal{H}_{0, s}\left(\mathbf{R}^{d}\right) \quad \text { or } \quad \mathcal{H}_{s}\left(\mathbf{R}^{d}\right)
$$

and has the Hermite expansion (2.10). If $a(x, D)$ is the creation or annihilation operator with respect to the coordinate $x_{j}$, then it follows by straight-forward computations that

$$
c_{\alpha}(a(x, D) f)=c_{\beta}(f),
$$

where

$$
\beta_{k}= \begin{cases}\alpha_{j} \pm 1, & k=j \\ \alpha_{k}, & k \neq j\end{cases}
$$

for some choice of plus or minus. By straight-forward computations it follows that if

$$
\left|c_{\alpha}(f)\right| \lesssim e^{-r|\alpha|^{\frac{1}{2 s}}} \quad \text { or } \quad\left|c_{\alpha}(f)\right| \lesssim r^{|\alpha|}(\alpha !)^{-\frac{1}{2}}
$$

for some or for every $r>0$, then $c_{\alpha}(x, D) f$ obeys the same type of estimate as $c_{\alpha}(f)$ does. Hence, the creation and annihilation operators are continuous on any of the spaces in (6.2).

The result now follows in general from the facts that any operator $a(x, D)$ with polynomial symbol is a superposition of the operators $f \mapsto$ $x_{j} \cdot f$ and $f \mapsto D_{j} f, j=1, \ldots, d$, and that any of the latter operators are linear combinations of creation and annihilation operators

Corollary 6.3. Let $s \in \overline{\mathbf{R}_{b}}$. Then the spaces in (3.2)-(3.5) and (4.7)(4.10) are invariant under multiplication of analytic polynomials and under complex differentiations.

Proof. The result follows from Proposition 6.2 and the fact that the creation and annihilation operators with respect to the coordinate $x_{j}$ carry over to multiplication and differentiation, respectively, with respect to the coordinate $z_{j}$, by the Bargmann transform.

Proposition 6.2 shows that several partial differential operators possess suitable mapping properties on Pilipović spaces. On the other hand, the following consequence of Theorem 4.3 shows that important properties, valid for other test function spaces and their distribution spaces, are violated. Here a test-function space $V$ and its dual $V^{\prime}$ are called test-function modules if the multiplication $(f, g) \mapsto f \cdot g$ is continuous from $V \times V$ to $V$, and thereby extendable to continuous mappings from $V^{\prime} \times V$ to $V$ and from $V \times V^{\prime}$ to $V$.

Proposition 6.4. Let $f$ be a Gauss function and let $s \in \overline{\mathbf{R}_{b}}$. Then the following is true: 
(1) $f \in \mathcal{H}_{0, \frac{1}{2}}\left(\mathbf{R}^{d}\right)$, if and only if $f(x)=C e^{-\frac{|x|^{2}}{2}+L(x)}$ for some (complex-valued) linear form $L$ on $\mathbf{R}^{d}$ and constant $C$;

(2) $\mathcal{H}_{0, s}\left(\mathbf{R}^{d}\right)$ and $\mathcal{H}_{0, s}^{\prime}\left(\mathbf{R}^{d}\right)$ are neither invariant under dilations nor test-function modules when $0<s \leq \frac{1}{2}$;

(3) $\mathcal{H}_{s}\left(\mathbf{R}^{d}\right)$ and $\mathcal{H}_{s}^{\prime}\left(\mathbf{R}^{d}\right)$ are neither invariant under dilations nor test-function modules when $0 \leq s<\frac{1}{2}$.

Proof. It suffices to prove (1). Let $\phi$ be as in (1.14), and set

$$
\phi_{A, L}(x)=e^{-\frac{1}{2}\langle A x, x\rangle+L(x)},
$$

when $A$ is a symmetric $d \times d$ matrix with positive real part, and $L$ is a linear form on $\mathbf{R}^{d}$ with values in $\mathbf{C}$. If $f=\phi_{I, L}$ for some (complexvalued) linear form $L$ on $\mathbf{R}^{d}$, then

$$
\left|\left(V_{\phi} f\right)(x, \xi)\right|=C_{0} e^{-\frac{1}{4}\left(\left|x-x_{0}\right|^{2}+\left|\xi-\xi_{0}\right|^{2}\right)},
$$

for some fixed $x_{0}, \xi_{0} \in \mathbf{R}^{d}$ and constant $C_{0}$. Since the right-hand side can be estimated by $C_{\varepsilon} e^{-\frac{1}{4}(1-\varepsilon)\left(|x|^{2}+|\xi|^{2}\right)}$, for every $\varepsilon>0$, it follows that $f \in \boldsymbol{\Sigma}\left(\mathbf{R}^{d}\right)$ in this case, in view of Remark 4.6.

Next assume that $f$ is a Gaussian and belongs to $\boldsymbol{\Sigma}\left(\mathbf{R}^{d}\right)$. Then $f=$ $C \phi_{A, L}$, for some constant $C$, symmetric $d \times d$ matrix $A$ with positive real part, and linear form $L$ on $\mathbf{R}^{d}$ with values in $\mathbf{C}$. Since $\boldsymbol{\Sigma}$ is invariant under the Fourier transform, it follows that

$$
\widehat{f}=C_{1} \phi_{A^{-1}, L_{0}}
$$

belongs to $\boldsymbol{\Sigma}\left(\mathbf{R}^{d}\right)$ for some choice of linear form $L_{0}$.

Let

$$
A_{1}=A(I+A)^{-1} \text { and } A_{2}=(I+A)^{-1} .
$$

By straight-forward computations we get

$$
\left(V_{\phi} f\right)(x, 0)=C_{1} \phi_{A, L} * \phi(x)=C_{2} \phi_{A_{1}, L_{1}}(x)
$$

and

$$
\left(V_{\phi} \widehat{f}\right)(\xi, 0)=C_{3} \phi_{A^{-1}, L_{0}} * \phi(\xi)=C_{4} \phi_{A_{2}, L_{2}}(\xi),
$$

for some constants $C_{1}, \ldots, C_{4}$ and linear forms $L_{1}$ and $L_{2}$. Since $f, \widehat{f} \in$ $\boldsymbol{\Sigma}\left(\mathbf{R}^{d}\right)$, Theorem 4.3, Remark 4.6 and the latter formulas imply that

$$
\operatorname{Re}\left(\left\langle A_{1} x, x\right\rangle\right) \geq \frac{|x|^{2}}{2} \text { and } \operatorname{Re}\left(\left\langle A_{2} x, x\right\rangle\right) \geq \frac{|x|^{2}}{2} .
$$

Hence, if $\lambda_{1}, \ldots, \lambda_{d}$ are the eigenvalues of $A$, it follows from the latter estimates and (6.3) that

$$
\operatorname{Re}\left(\left(1+\lambda_{j}\right)^{-1}\right) \geq 2^{-1} \text { and } \operatorname{Re}\left(\left(1+\lambda_{j}^{-1}\right)^{-1}\right) \geq 2^{-1}
$$

when $j=1, \ldots, d$, which is true only when $\lambda_{j}=1$ for every $j$. 
In fact, by letting $s_{j}$ and $t_{j}$ be the real and imaginary parts of $\lambda_{j}$, the latter inequalities become

$$
s_{j}^{2}+t_{j}^{2} \leq 1 \leq s_{j}^{2}-3 t_{j}^{2}
$$

which implies that $s_{j}= \pm 1$ and $t_{j}=0$. Since $s_{j}>0$, it follows that $\lambda_{j}=s_{j}=1$, and the result follows.

6.3. A broader class of Pilipović spaces. The mapping properties explained in Theorem 4.3 can serve as a source of inspiration of defining broad families of function and distribution spaces, related to Pilipović spaces and their spaces of distributions.

In what follows, let $\kappa_{1}$ and $\kappa_{2}$ be given by (3.1), and let

$$
\vartheta_{r, s}(x)=\left\{\begin{array}{ll}
e^{r(\log \langle x\rangle)^{\frac{1}{1-2 s}}}, & s<\frac{1}{2}, \\
e^{r|x|^{\kappa_{1}(\sigma)}}, & s=b_{\sigma}, \\
e^{\frac{r|x|^{2}}{2(1+r)}}, & s=\frac{1}{2}, \\
e^{\frac{|x|^{2}}{2}-r|x|^{\frac{1}{s}}}, & s>\frac{1}{2},
\end{array} \quad \vartheta_{r, s}^{*}(x)= \begin{cases}e^{r|x|^{\kappa_{2}(\sigma)}}, & s=b_{\sigma}, \\
e^{\frac{(1+r)|x|^{2}}{2 r}}, & s=\frac{1}{2}, \\
e^{\frac{|x|^{2}}{2}+r|x|^{\frac{1}{s}}}, & s>\frac{1}{2},\end{cases}\right.
$$

$$
\omega_{r, s, t}(z)=\vartheta_{r, t}(x) \vartheta_{r, s}(\xi), \quad \text { when } s, t \in \overline{\mathbf{R}_{b}}, z=x+i \xi, x, \xi \in \mathbf{R}^{d},
$$

and

$$
\omega_{r, s, t}^{*}(z)=\vartheta_{r, t}^{*}(x) \vartheta_{r, s}^{*}(\xi), \quad \text { when } s, t \in \overline{\mathbf{R}_{b}}, \quad z=x+i \xi, x, \xi \in \mathbf{R}^{d} .
$$

Furthermore, let

$$
\begin{aligned}
\mathcal{A}_{t}^{s}\left(\mathbf{C}^{d}\right) & =\left\{F \in A\left(\mathbf{C}^{d}\right) ;|F(z)| \lesssim \omega_{r, s, t}(z), \text { for some } r>0\right\}, \\
\mathcal{A}_{0, t}^{s}\left(\mathbf{C}^{d}\right) & =\left\{F \in A\left(\mathbf{C}^{d}\right) ;|F(z)| \lesssim \omega_{r, s, t}(z), \text { for every } r>0\right\},
\end{aligned}
$$

and let $\left(\mathcal{A}_{t}^{s}\right)^{\prime}\left(\mathbf{C}^{d}\right)$ and $\left(\mathcal{A}_{0, t}^{s}\right)^{\prime}\left(\mathbf{C}^{d}\right)$ be the duals of $\mathcal{A}_{t}^{s}\left(\mathbf{C}^{d}\right)$ and $\mathcal{A}_{0, t}^{s}\left(\mathbf{C}^{d}\right)$, respectively, $s, t \in \overline{\mathbf{R}_{b}}$. Then

$$
\left(\mathcal{A}_{t}^{s}\right)^{\prime}\left(\mathbf{C}^{d}\right)=\left\{F \in A\left(\mathbf{C}^{d}\right) ;|F(z)| \lesssim \omega_{r, s, t}^{*}(z) \text {, for every } r>0\right\}, s, t>b_{1}
$$

and

$$
\left(\mathcal{A}_{0, t}^{s}\right)^{\prime}\left(\mathbf{C}^{d}\right)=\left\{F \in A\left(\mathbf{C}^{d}\right) ;|F(z)| \lesssim \omega_{r, s, t}^{*}(z) \text {, for some } r>0\right\}, s, t>b_{1} \text {. }
$$

We now let $\mathcal{S}_{t}^{s}\left(\mathbf{R}^{d}\right), \boldsymbol{\Sigma}_{t}^{s}\left(\mathbf{R}^{d}\right),\left(\mathcal{S}_{t}^{s}\right)^{\prime}\left(\mathbf{R}^{d}\right)$ and $\left(\boldsymbol{\Sigma}_{t}^{s}\right)^{\prime}\left(\mathbf{R}^{d}\right)$ be the counter images of the latter spaces, i. e.,

$$
\begin{aligned}
\mathcal{S}_{t}^{s}\left(\mathbf{R}^{d}\right) & \equiv \mathfrak{V}_{d}^{-1}\left(\mathcal{A}_{t}^{s}\left(\mathbf{C}^{d}\right)\right), & \boldsymbol{\Sigma}_{t}^{s}\left(\mathbf{R}^{d}\right) & \equiv \mathfrak{V}_{d}^{-1}\left(\mathcal{A}_{0, t}^{s}\left(\mathbf{C}^{d}\right)\right), \\
\left(\mathcal{S}_{t}^{s}\right)^{\prime}\left(\mathbf{R}^{d}\right) & \equiv \mathfrak{V}_{d}^{-1}\left(\left(\mathcal{A}_{t}^{s}\right)^{\prime}\left(\mathbf{C}^{d}\right)\right), & \left(\boldsymbol{\Sigma}_{t}^{s}\right)^{\prime}\left(\mathbf{R}^{d}\right) & \equiv \mathfrak{V}_{d}^{-1}\left(\left(\mathcal{A}_{0, t}^{s}\right)^{\prime}\left(\mathbf{C}^{d}\right)\right),
\end{aligned}
$$

for suitable $s$ and $t$.

Evidently, from the definitions it follows that

$$
\mathcal{S}_{s}^{s}=\mathcal{S}_{s}, \quad \Sigma_{s}^{s}=\Sigma_{s}, \quad\left(\mathcal{S}_{s}^{s}\right)^{\prime}=\mathcal{S}_{s}^{\prime} \quad \text { and } \quad\left(\Sigma_{s}^{s}\right)^{\prime}=\Sigma_{s}^{\prime},
$$


for admissible $s \in \overline{\mathbf{R}_{b}}$, and

$$
\mathcal{S}_{s}^{t}=\mathcal{S}_{s}^{t}, \quad \Sigma_{s}^{t}=\Sigma_{s}^{t}, \quad\left(\mathcal{S}_{s}^{t}\right)^{\prime}=\left(\mathcal{S}_{s}^{t}\right)^{\prime} \quad \text { and } \quad\left(\Sigma_{s}^{t}\right)^{\prime}=\left(\Sigma_{s}^{t}\right)^{\prime},
$$

when the spaces on the right-hand sides are non-trivial. An interesting question concerns of finding convenient descriptions of the spaces in (6.4). This seems to be non-trivial from the point of view of Hermite series expansions, in view of [14].

By the link between the Bargmann transform and short-time Fourier transform (1.24) when $\phi$ is given by (1.14), it follows that we may identify the spaces in (6.4) by suitable estimates on the involved elements. More precisely, let $\widetilde{\vartheta}_{r, s}$ and $\widetilde{\vartheta}_{r, s}^{*}$ be the modifications of $\vartheta_{r, s}$ and $\vartheta_{r, s}^{*}$, given by

$$
\widetilde{\vartheta}_{r, s}(x)= \begin{cases}e^{-\frac{|x|^{2}}{4}+r(\log \langle x\rangle)^{\frac{1}{1-2 s}}}, & s<\frac{1}{2}, \\ e^{-\frac{|x|^{2}}{4}+r|x|^{\kappa_{1}(\sigma)}}, & s=b_{\sigma}, \\ e^{\frac{|x|^{2}}{4(1+r)}}, & s=\frac{1}{2}, \\ e^{-r|x|^{\frac{1}{s}}}, & s>\frac{1}{2},\end{cases}
$$

and

$$
\widetilde{\vartheta}_{r, s}^{*}(x)= \begin{cases}e^{-\frac{|x|^{2}}{4}+r|x|^{\kappa_{2}(\sigma)}}, & s=b_{\sigma}, \\ e^{r|x|^{\frac{1}{s}}}, & s \geq \frac{1}{2},\end{cases}
$$

then we get the following.

Proposition 6.5. Let $r>0, \kappa_{1} \kappa_{2}$ and $\phi$ be as in (1.14) and (3.1), $\widetilde{\vartheta}_{r, s}$ for $s \in \overline{\mathbf{R}_{b}}$ and $\widetilde{\vartheta}_{r, s}^{*}$ for $s \in \mathbf{R}_{b}$ be given by (6.5) and (16.6), and let

$$
\omega_{r, s, t}(x, \xi) \equiv \widetilde{\vartheta}_{r, t}(x) \widetilde{\vartheta}_{r, s}(\xi) \quad \text { and } \quad \omega_{r, s, t}^{*}(x, \xi) \equiv \widetilde{\vartheta}_{r, t}^{*}(x) \widetilde{\vartheta}_{r, s}^{*}(\xi) .
$$

Then the following is true:

(1) if $s, t \geq 0(s, t>0)$, then $f \in \mathcal{S}_{t}^{s}\left(\mathbf{R}^{d}\right)\left(f \in \boldsymbol{\Sigma}_{t}^{s}\left(\mathbf{R}^{d}\right)\right)$, if and only if

$$
\left|\left(V_{\phi} f\right)(x, \xi)\right| \lesssim \omega_{r, s, t}(x, \xi)
$$

for some (for every) $r>0$;

(2) if $s, t>b_{1}$, then $f \in\left(\mathcal{S}_{t}^{s}\right)^{\prime}\left(\mathbf{R}^{d}\right)\left(f \in\left(\boldsymbol{\Sigma}_{t}^{s}\right)^{\prime}\left(\mathbf{R}^{d}\right)\right)$, if and only if

$$
\left|\left(V_{\phi} f\right)(x, \xi)\right| \lesssim \omega_{r, s, t}^{*}(x, \xi)
$$

for every (for some) $r>0$. 
6.4. Tables of properties for function and distribution spaces. In the following tables we list the boundedness properties of the spaces considered in previous sections. Here we let $N_{1}=\frac{N}{\log N}$. The first tabular lists the test function spaces and their properties.

\begin{tabular}{l||c|c|c|c}
$\mathcal{H}_{s}\left(\mathbf{R}^{d}\right) / \mathcal{H}_{0, s}\left(\mathbf{R}^{d}\right)$ & $\left|H^{N} f\right| \lesssim$ & $\left|c_{\alpha}(f)\right| \lesssim$ & $\left|\mathfrak{V}_{d} f(z)\right| \lesssim$ & References \\
\hline$s>\frac{1}{2}$ & $R^{N} N !^{2 s}$ & $e^{-R|\alpha|^{\frac{1}{2 s}}}$ & $e^{\frac{|z|^{2}}{2}-R|z|^{\frac{1}{s}}}$ & Thm. [5.2] \\
$\mathcal{H}_{\frac{1}{2}}\left(\mathbf{R}^{d}\right)$ & $R^{N} N !$ & $e^{-R|\alpha|}$ & $e^{(1-R)^{\frac{|z|^{2}}{2}}}$ & Thm. [5.2 \\
$\mathcal{H}_{0, \frac{1}{2}}\left(\mathbf{R}^{d}\right)$ & $R^{N} N !$ & $e^{-R|\alpha|}$ & $e^{R|z|^{2}}$ & Thm. 4.3 \\
$s=b_{\sigma}, \sigma<\infty$ & $\operatorname{Se}^{3}$ & $R^{|\alpha|}(\alpha !)^{-\frac{1}{2 \sigma}}$ & $e^{R|z|^{\frac{2 \sigma}{\sigma+1}}}$ & Thm. 3.1 \\
$s<\frac{1}{2}$ & $R^{N} N !^{2 s}$ & $e^{-R|\alpha|^{\frac{1}{2 s}}}$ & $e^{R(\log \langle z\rangle)^{\frac{1}{1-2 s}}}$ & Thm. 4.3!
\end{tabular}

Table 1: The test function spaces $\mathcal{H}_{s}\left(\mathbf{R}^{d}\right)$ and $\mathcal{H}_{0, s}\left(\mathbf{R}^{d}\right)$. For $\mathcal{H}_{s}\left(\mathcal{H}_{0, s}\right)$, the above estimates should hold for some (for every) $R>0$.

\begin{tabular}{l||c|c|c}
$\mathcal{H}_{s}^{\prime}\left(\mathbf{R}^{d}\right) / \mathcal{H}_{0, s}^{\prime}\left(\mathbf{R}^{d}\right)$ & $\left|c_{\alpha}(f)\right| \lesssim$ & $\left|\mathfrak{V}_{d} f(z)\right| \lesssim$ & References \\
\hline$s>\frac{1}{2}$ & $e^{R|\alpha|^{\frac{1}{2 s}}}$ & $e^{\frac{|z|^{2}}{2}+R|z|^{\frac{1}{s}}}$ & Thm. [5.2 \\
$\mathcal{H}_{\frac{1}{2}}^{\prime}\left(\mathbf{R}^{d}\right)$ & $e^{R|\alpha|}$ & $e^{(1+R) \frac{|z|^{2}}{2}}$ & Thm. [5.2 \\
$\mathcal{H}_{0, \frac{1}{2}}^{\prime}\left(\mathbf{R}^{d}\right)$ & $e^{R|\alpha|}$ & $e^{R|z|^{2}}$ & Thm. 3.2 \\
$s=b_{\sigma}, \sigma>1$ & $R^{|\alpha|}(\alpha !)^{\frac{1}{2 \sigma}}$ & $e^{R|z|^{\frac{2 \sigma}{\sigma-1}}}$ & Thm. 3.2 \\
$s=b_{\sigma}, \sigma \leq 1$ & $R^{|\alpha|}(\alpha !)^{\frac{1}{2 \sigma}}$ & No conditions & Thm. 3.2 \\
$s<\frac{1}{2}$ & $e^{-R|\alpha|^{\frac{1}{2 s}}}$ & No conditions & Thm. 4.4
\end{tabular}

Table 2: The distribution spaces $\mathcal{H}_{s}^{\prime}\left(\mathbf{R}^{d}\right)$ and $\mathcal{H}_{0, s}^{\prime}\left(\mathbf{R}^{d}\right)$. For $\mathcal{H}_{s}^{\prime}\left(\mathcal{H}_{0, s}^{\prime}\right)$, the above estimates should hold for every (for some) $R>0$.

\section{REFERENCES}

[1] V. Bargmann On a Hilbert space of analytic functions and an associated integral transform, Comm. Pure Appl. Math., 14 (1961), 187-214.

[2] V. Bargmann On a Hilbert space of analytic functions and an associated integral transform. Part II. A family of related function spaces. Application to distribution theory., Comm. Pure Appl. Math., 20 (1967), 1-101.

${ }^{2}$ See also 4 .

${ }^{3}$ If $\sigma=1$, then $f$ should here fulfill $\left|H^{N} f\right| \lesssim R^{N_{1}} N_{1}^{N-N_{1}}$ (cf. [9]).

${ }^{4}$ See also [9]. 
[3] P. Boggiatto, J. Toft Embeddings and compactness for generalized SobolevShubin spaces and modulation spaces, Appl. Anal. (3) 84 (2005), 269-282.

[4] M. Cappiello, L. Rodino, J. Toft Radial symmetric elements and the Bargmann transform, Integral Transform. Spec. Funct. 25 (2014), 756-764.

[5] J. Chung, S.-Y. Chung, D. Kim, Characterizations of the Gelfand-Shilov spaces via Fourier transforms, Proc. Amer. Math. Soc. 124 (1996), 2101-2108.

[6] A. Dasgupta, M. Ruzhansky Gevrey functions and ultradistributions on compact Lie groups and homogeneous spaces, Bull. Sci. Math. 138 (2014), 756-782.

[7] S. J. L. Eijndhoven Functional analytic characterizations of the Gelfand-Shilov spaces $S_{\alpha}^{\beta}$, Nederl. Akad. Wetensch. Indag. Math. 49 (1987), 133-144.

[8] H. G. Feichtinger Modulation spaces on locally compact abelian groups. Technical report, University of Vienna, Vienna, 1983; also in: M. Krishna, R. Radha, S. Thangavelu (Eds) Wavelets and their applications, Allied Publishers Private Limited, NewDehli Mumbai Kolkata Chennai Hagpur Ahmedabad Bangalore Hyderbad Lucknow, 2003, pp. 99-140.

[9] C. Fernandez, A. Galbis, J. Toft The Bargmann transform and powers of harmonic oscillator on Gelfand-Shilov subspaces, RACSAM (to appear, see also arXiv:1507.04850).

[10] H. G. Feichtinger, K. H. Gröchenig, D. Walnut Wilson bases and modulation spaces, Math. Nach. 155 (1992), 7-17.

[11] I. M. Gelfand, G. E. Shilov, Generalized functions, II-III, Academic Press, NewYork London, 1968.

[12] T. Gramchev, S. Pilipović, L. Rodino Classes of degenerate elliptic operators in Gelfand-Shilov spaces in: L. Rodino, M. W. Wong (eds) New developments in pseudo-differential operators, Operator Theory: Advances and Applications 189, Birkhäuser, Basel, 2009, pp. 15-31.

[13] T. Gramchev, S. Pilipović, L. Rodino Eigenfunction expansions in $\mathbb{R}^{n}$, Proc. Amer. Math. Soc. 139 (2011), 4361-4368.

[14] T. Gramchev, A. Lecke, S. Pilipović, L. Rodino Gelfand-Shilov type spaces through Hermite expansions in: S. Pilipović, J. Toft (eds) Pseudo-Differential Operators and Generalized Functions, Operator Theory: Advances and Applications 245, Birkhäuser, Basel Heidelberg NewYork Dordrecht London, 2015, pp. 95-105.

[15] K. H. Gröchenig Foundations of Time-Frequency Analysis, Birkhäuser, Boston, 2001.

[16] K. Gröchenig, G. Zimmermann Spaces of test functions via the STFT J. Funct. Spaces Appl. 2 (2004), 25-53.

[17] L. Hörmander The Analysis of Linear Partial Differential Operators, vol I-III, Springer-Verlag, Berlin Heidelberg NewYork Tokyo, 1983, 1985.

[18] A.M.E.M. Janssen, S.J.L. Eijndhoven Spaces of type W, growth of Hermite coefficients, Wigner distribution, and Bargmann transform, J. Math. Anal. Appl. 152 (1990), 368-390.

[19] Z. Lozanov Crvenković, D. Perišić Hermite expansions of elements of Gelfand Shilov spaces in quasianalytic and non quasianalytic case, Novi Sad J. Math. 37 (2007), 129-147.

[20] F. Nicola, L. Rodino Global pseudo-differential calculus on Euclidean spaces, Pseudo-Differential Operators. Theory and Applications 4 Birkhäuser Verlag, Basel, 2010.

[21] S. Pilipović Generalization of Zemanian spaces of generalized functions which have orthonormal series expansions, SIAM J. Math. Anal. 17 (1986), 477-484.

[22] S. Pilipović Tempered ultradistributions, Boll. U.M.I. 7 (1988), 235-251. 
[23] M. Signahl, J. Toft Mapping properties for the Bargmann transform on modulation spaces, J. Pseudo-Differ. Oper. Appl. 3 (2012), 1-30.

[24] N. Teofanov Ultradistributions and time-frequency analysis. In "Pseudodifferential operators and related topics", 173-192, Oper. Theory Adv. Appl., 164, Birkhäuser, Basel, 2006.

[25] J. Toft Continuity properties for modulation spaces with applications to pseudodifferential calculus, II, Ann. Global Anal. Geom., 26 (2004), 73-106.

[26] J. Toft The Bargmann transform on modulation and Gelfand-Shilov spaces, with applications to Toeplitz and pseudo-differential operators, J. PseudoDiffer. Oper. Appl. 3 (2012), 145-227.

[27] J. Toft Multiplication properties in Gelfand-Shilov pseudo-differential calculus in: S. Molahajlo, S. Pilipović, J. Toft, M. W. Wong (eds) Pseudo-Differential Operators, Generalized Functions and Asymptotics, Operator Theory: Advances and Applications 231, Birkhäuser, Basel Heidelberg NewYork Dordrecht London, 2013, pp. 117-172.

[28] A. Zayed, A. Garcia New sampling formulae for the fractional Fourier transform, Signal processing 77 (1999), 111-114.

Department of Mathematics, Linnæus University, VÄXjö, Sweden

E-mail address: joachim.toft@lnu.se 